\title{
Reflection systems and partial root systems
}

\author{
Ottmar Loos and Erhard Neher \\ Communicated by Karl-Hermann Neeb
}

\begin{abstract}
We develop a general theory of reflection systems and, more specifically, partial root systems which provide a unifying framework for finite root systems, Kac-Moody root systems, extended affine root systems and various generalizations thereof. Nilpotent and prenilpotent subsets are studied in this setting, based on commutator sets and the descending central series. We show that our notion of a prenilpotent pair coincides, for Kac-Moody root systems, with the one defined by Tits in terms of positive systems and the Weyl group.
\end{abstract}

Keywords. Reflection systems, partial root systems, affine root systems, extended affine root systems, nilpotent sets of roots.

2000 Mathematics Subject Classification. 17B20, 17B67, 17 B99.

\section{Introduction}

Root systems provide a powerful framework for dealing with combinatorial questions arising in the structure and representation theory of Lie algebras, the classical case being finite-dimensional semisimple Lie algebras and finite root systems [14]. The same is true for various generalizations of semisimple Lie algebras and appropriately defined root systems. Without any claim of completeness, we mention some examples. Locally finite root systems [26] arise in Lie algebras of infinite rank, for example the affine Lie algebras of infinite rank [23, 7.11], Lie algebras graded by infinite root systems [33] or split locally finite Lie algebras [30, 31, 41]. Kac-Moody Lie algebras give rise to root systems with the same name [23, 28], and generalizations of Kac-Moody root systems, called sets of root data, have been used to describe subsystems and correspondingly certain subalgebras of Kac-Moody algebras [28, Chapter 5]. Extended affine Lie algebras, which generalize affine Lie algebras without necessarily being themselves Kac-Moody algebras, require extended affine root systems already in their definition [1], and generalizations of extended affine root systems have come up naturally in the struc-

The research for this paper was partially supported by the second-named author's NSERC Discovery grant. 
ture theory of extended affine Lie algebras presently developed, see for example [8, 5, 6], [29], [48] and the closely related root systems appearing in [37] and [38]. An axiom system for the root systems of the basic classical Lie superalgebras is described in [39], and for the root systems of Borcherds' generalization of KacMoody algebras in [11, 12].

In the same vein, root systems are an important ingredient in the structure theory of reductive algebraic groups, algebraic groups over local fields ([15], see §4.3) or Kac-Moody groups [18, 24, 36, 43].

Looking at this list of examples of root systems, one notices that despite the sometimes substantial differences there are some basic features common to all of them. Yet there does not exist a general theory of root systems. One of the aims of this paper is therefore

- to create a general framework for studying root systems, encompassing all the examples mentioned above. We call these new objects partial root systems.

Our motivation for doing so goes beyond just providing a new setting for root systems. In fact, this paper grew out of an attempt to define groups "à la Steinberg"a terminology due to Tits [43] —in a general category of groups with commutator relations, generalizing Kac-Moody groups. One of the special features of KacMoody root systems is that they come equipped with a distinguished set of simple and positive roots. This is no longer the case for, say, extended affine root systems or locally finite root systems, where there always exist many (in fact, too many) sets of positive roots and in general no simple roots at all. Since the distinguished set of positive roots in a Kac-Moody root system is used to define prenilpotent pairs, which are essential for Tits' approach to Kac-Moody groups, one is faced with the problem of finding a good concept of prenilpotent pairs which works in general without reference to a distinguished positive system. A second aim of this paper is therefore

- to study nilpotent subsets of partial root systems; in particular, prenilpotent pairs.

Among partial root systems, the class of extended affine root systems [1] has recently attracted much interest. There are presently many similar approaches with sometimes conflicting terminology. As a third aim of this paper, we intend

- to put the theory of extended affine root systems and similar structures in a bigger perspective by viewing them as extensions in an appropriate sense.

To achieve these goals we work on three levels of generality. They are, in decreasing order: subsets of torsion-free abelian groups, reflection systems, and partial root systems. 
In Section 1 we consider pairs $(R, X)$ where $X$ is a torsion-free abelian group and $R$ is a subset of $X$ which contains 0 and generates $X$. We study nilpotent subsets in this framework, using appropriate definitions of the descending and ascending series, very much in analogy to group theory.

The notion of a reflection system, developed in Section 2, is meant to capture the essence of a consistent assignment of hyperplane reflections to some of the elements of $R$ as follows: Let $X$ be a vector space over a field of characteristic 0 and let $R \subset X$ be a spanning set with $0 \in R$. Suppose $s \mapsto s_{\alpha}$ is a map assigning to each $\alpha \in R$ a hyperplane reflection $s_{\alpha}$ or the identity on $X$. We put $R^{\mathrm{im}}:=\left\{\alpha \in R: s_{\alpha}=\mathrm{Id}\right\}$ and $R^{\mathrm{re}}:=R \backslash R^{\mathrm{im}}$, and call their elements imaginary roots and reflective roots, respectively. This terminology is of course suggested by the example of root systems of Kac-Moody Lie algebras where the reflective roots are just the real roots. The triple $(R, X, s)$ is called a reflection system if the following axioms hold:

- $\alpha \in R^{\text {re }}$ implies $\alpha \neq s_{\alpha}(\alpha)=-\alpha \in R^{\text {re }}$, in particular $0 \in R^{\mathrm{im}}$,

- $s_{\alpha}(\beta) \in R$ and $s_{s_{\alpha}(\beta)}=s_{\alpha} s_{\beta} s_{\alpha}$, for all $\alpha, \beta \in R$,

- $s_{c \alpha}=s_{\alpha}$ whenever $c \in \mathbb{K}^{\times}$and both $\alpha$ and $c \alpha$ belong to $R^{\text {re }}$.

We also introduce pre-reflection systems, defined by the first and a weak version of the second axiom. As usual, $s_{\alpha}$ is given by the formula

$$
s_{\alpha}(x)=x-\left\langle x, \alpha^{\vee}\right\rangle \alpha
$$

where $\alpha^{\vee}$ is a linear form on $X$. It is possible to formulate the axioms in terms of the map $\alpha \mapsto \alpha^{\vee}$ (§2.3). Observe that we do not assume $R$ or even $R^{\text {re }}$ to be reduced in the sense that $\alpha, c \alpha \in R^{\text {re }}$ implies $c= \pm 1$, nor that the Cartan numbers $\left\langle\beta, \alpha^{\vee}\right\rangle$ be integers, although this condition will be introduced later. Also, in the Bourbaki tradition [14] and unlike most of the papers quoted above, we do not a priori require the existence of an invariant bilinear form, although such forms do play an important role in special situations, in particular, for affine reflection systems (Section 5).

In Section 3 we introduce partial root systems. These are reflection systems with the following additional properties:

(PRS1) The Cartan numbers are integers and, for all $\alpha, \beta \in R^{\text {re }}, \operatorname{sgn}\left\langle\alpha, \beta^{\vee}\right\rangle=$ $\operatorname{sgn}\left\langle\beta, \alpha^{\vee}\right\rangle$,

(PRS2) for all $\alpha \in R^{\mathrm{re}}, \beta \in R$, the root string $R \cap(\beta+\mathbb{Z} \alpha)$ is finite and without gaps,

(PRS3) for all $\alpha, \beta \in R^{\mathrm{re}},\left\langle\beta, \alpha^{\vee}\right\rangle \geq 0$ and $\alpha+\beta \in R$ imply $\alpha+\beta \in R^{\mathrm{re}}$. 
We compare the notion of a prenilpotent set $A$, defined as in Section 1 by means of the descending central series, with that introduced by Tits in the setting of KacMoody algebras, which postulates the existence of Weyl group elements mapping $A$ to $P$ and $-P$, respectively, where $P$ is the standard set of positive roots. The main result here is Theorem 3.7, which asserts the equivalence of the two notions of prenilpotence for partial root systems in case $A$ has cardinality at most two and $P$ is a positive system of scalar type. The latter condition is satisfied by the standard positive systems of Kac-Moody algebras, so our result is applicable in this important special case and hence gives a new approach to the concept of nilpotent subsets of Kac-Moody roots (Corollary 3.8).

The last two sections deal with extensions and affine reflection systems. An extension is a morphism $f:(R, X) \rightarrow(S, Y)$ of reflection systems satisfying $f\left(R^{\mathrm{re}}\right)=S^{\mathrm{re}}$ and $f\left(R^{\mathrm{im}}\right)=S^{\mathrm{im}}$. Of particular interest is the case where $S$ is nondegenerate in the sense that $\bigcap_{\beta \in S} \beta^{\vee}=0$, because then $S$ is uniquely determined by $R$. We describe extensions by means of extension data on $S$ in Theorem 4.6, and give necessary and sufficient conditions for $R$ to be reduced or a partial root system in Corollary 4.7 .

An affine reflection system is defined as an extension of a locally finite root system. Such systems can also be characterized using affine forms (Proposition 5.4), and contain as special cases extended affine root systems and their many generalizations (\$5.3).

We plan to use the results obtained here to study groups "à la Steinberg" in a general category of groups with commutator relations.

\section{Nilpotent sets of roots}

\subsection{Closed and positive subsets}

Let $X$ be a torsion-free abelian group. For a subset $A \subset X$, let $\mathbb{N}^{(A)}$ be the free abelian monoid generated by $A$, i.e., the set of all maps $v: A \rightarrow \mathbb{N}$ which are zero except for finitely many $\alpha \in A$. Depending on the context, it may be more convenient to think of an element of $\mathbb{N}^{(A)}$ as a family $\left(n_{\alpha}\right)_{\alpha \in A}$, where $n_{\alpha} \in \mathbb{N}$ and $n_{\alpha}=0$ except for finitely many $\alpha$. We denote by $\kappa: \mathbb{N}^{(A)} \rightarrow X$ the canonical map sending $v$ to $\sum_{\alpha \in A} v(\alpha) \alpha$ and put

$$
\begin{aligned}
\mathbb{N}[A] & :=\kappa\left(\mathbb{N}^{(A)}\right) \quad \text { and } \\
\mathbb{N}_{+}[A]: & =\kappa\left(\mathbb{N}^{(A)} \backslash\{0\}\right)=\bigcup_{n=1}^{\infty} \underbrace{(A+\cdots+A)}_{n} .
\end{aligned}
$$


We introduce the category SG, whose objects are pairs $(R, X)$ consisting of a torsion-free abelian group $X$ and a subset $R \subset X$ which generates $X$ as abelian group and satisfies $0 \in R$. The morphisms $f:(R, X) \rightarrow(S, Y)$ of $\mathbf{S G}$ are the group homomorphisms $f: X \rightarrow Y$ satisfying $f(R) \subset S$.

Let $(R, X) \in$ SG. Generalizing a concept of [26, 10.2], a subset $C \subset R$ is called additively closed (or simply closed if there is no ambiguity) if $C=$ $R \cap \mathbb{N}_{+}[C]$, i.e., if for all $\alpha_{1}, \ldots, \alpha_{n} \in C$ with $\beta:=\alpha_{1}+\cdots+\alpha_{n} \in R$, we have $\beta \in C$. The additive closure $A^{c}$ of a subset $A$ of $R$ is the smallest additively closed subset containing $A$; it is given by

$$
A^{c}=R \cap \mathbb{N}_{+}[A] .
$$

In the special case $A=\{\alpha, \beta\}$, we write

$$
[\alpha, \beta]:=\{\alpha, \beta\}^{c}
$$

and call it the closed root interval between $\alpha$ and $\beta$. If $f:(R, X) \rightarrow\left(R^{\prime}, X^{\prime}\right)$ is a morphism of $\mathbf{S G}$, then

$$
f\left(A^{c}\right) \subset f(A)^{c} .
$$

This is immediate from the definitions.

A subset $A$ of $R$ is called positive if it is additively closed and $A \cap(-A) \subset\{0\}$. We will say a subset $A$ of $R$ is strictly positive if it is positive and $0 \notin A$. Obviously,

$$
A \text { is strictly positive } \Longleftrightarrow A \text { is closed and } 0 \notin A \text {. }
$$

For any subset $A$ of $R$ we put $A^{\times}:=A \backslash\{0\}$. Then one shows as in [26, Lemma $10.10(\mathrm{a})]$ that

$$
\begin{aligned}
A \text { is positive } & \Longleftrightarrow A^{\times} \text {is strictly positive } \\
& \Longleftrightarrow A \text { is closed and } \mathbb{N}_{+}[A] \cap \mathbb{N}_{+}[-A] \subset\{0\} .
\end{aligned}
$$

Using (1.6) one sees that a positive subset $A$ defines a partial order $\succeq_{A}$ on the additive group of $X$ by

$$
x \succeq_{A} y \Longleftrightarrow x-y \in \mathbb{N}[A]
$$

for which $A \cup\{0\}=\left\{\alpha \in R: \alpha \succeq_{A} 0\right\}$ and $A^{\times}=\left\{\alpha \in R: \alpha \succ_{A} 0\right\}$. Here the notation $x \succ_{A} y$ means $x \succeq_{A} y$ and $x \neq y$. 
The following characterization of strictly positive subsets justifies our terminology.

Lemma 1.1. Let $(R, X) \in \mathbf{S G}$, and let $\alpha, \beta \in R^{\times}$. Then $\{\alpha, \beta\}^{c}$ is strictly positive, i.e., $0 \notin\{\alpha, \beta\}^{c}$, if and only if there exists a function $h: \mathbb{N}_{+}[\{\alpha, \beta\}] \rightarrow \mathbb{N}_{+}$ satisfying $h(\gamma+\delta)=h(\gamma)+h(\delta)$ for all $\gamma, \delta \in \mathbb{N}_{+}[\{\alpha, \beta\}]$.

A function $h$ as above is called a height function.

Proof. If $h$ exists then obviously $0 \notin\{\alpha, \beta\}^{c}$. Conversely, assume that $0 \notin\{\alpha, \beta\}^{c}$. Recall that any $\gamma \in P:=\mathbb{N}_{+}[\{\alpha, \beta\}]$ can be written in the form $\gamma=m \alpha+n \beta$. Hence, for any choice of $p, r \in \mathbb{N}_{+}$we obtain a function $h: P \rightarrow \mathbb{N}_{+}$with the desired properties by putting

$$
h(m \alpha+n \beta)=m r+n p,
$$

as long as this function is well-defined. This is of course the case if $\alpha, \beta$ are $\mathbb{Z}$-free. Otherwise, there exist $p, q \in \mathbb{Z},(p, q) \neq(0,0)$, such that $p \alpha+q \beta=0$. If $p=0$ then $q \beta=0$ yields the contradiction $q=0$ or $\beta=0$. We can therefore assume that $p \neq 0$ and $q \neq 0$. Since $0 \notin P, p$ and $q$ have different signs. Without loss of generality we can assume $p>0>q=-r$. To prove that with these $p$ and $r$ the function $h$ of (1.8) is well-defined, we suppose that $\gamma \in P$ can be written in the form $\gamma=m \alpha+n \beta$ and $\gamma=m^{\prime} \alpha+n^{\prime} \beta$ with $m, m^{\prime}, n, n^{\prime} \in \mathbb{N}$ and show that

$$
m r+n p=m^{\prime} r+n^{\prime} p .
$$

Indeed, multiplying $\left(m-m^{\prime}\right) \alpha=\left(n^{\prime}-n\right) \beta$ by $p$ and replacing $p \alpha$ by $r \beta$ shows $\left(m-m^{\prime}\right) r \beta=\left(n^{\prime}-n\right) p \beta$, from which (1.9) immediately follows.

\subsection{Commutator sets}

Let $(R, X) \in \mathbf{S G}$. For arbitrary subsets $A, B$ of $R$ we define the commutator set

$$
(A, B):=R \cap\left(\mathbb{N}_{+}[A]+\mathbb{N}_{+}[B]\right) .
$$

Thus $\gamma \in(A, B)$ if and only if $\gamma$ belongs to $R$ and has the form

$$
\gamma=\alpha_{1}+\cdots+\alpha_{p}+\beta_{1}+\cdots+\beta_{q}
$$

where $p, q \geq 1, \alpha_{i} \in A$, and $\beta_{j} \in B$.

If $A=\{\alpha\}$ consists of a single element, we simply write $(\alpha, B)$ instead of $(\{\alpha\}, B)$, and similarly

$$
(\alpha, \beta):=(\{\alpha\},\{\beta\})=R \cap\left(\mathbb{N}_{+} \alpha+\mathbb{N}_{+} \beta\right),
$$


called the open root interval from $\alpha$ to $\beta$. The following properties follow easily from the definition:

$$
\begin{aligned}
(A, \emptyset)=\emptyset, & A \cup(A, A)=A^{c}=(A, 0), \\
A \text { is closed } & \Longleftrightarrow(A, A) \subset A, \\
0 \in B^{c} & \Longrightarrow A^{c} \subset(A, B), \\
(A, B)=(B, A) & =\left(A^{c}, B\right)=\left(A^{c}, B^{c}\right)=(A, B)^{c}, \\
A^{\prime} \subset A, B^{\prime} \subset B & \Longrightarrow\left(A^{\prime}, B^{\prime}\right) \subset(A, B), \\
(A \cup B)^{c} & =A^{c} \cup(A, B) \cup B^{c}, \\
(A,(A, B)) & \subset(A, B) .
\end{aligned}
$$

If $f:(R, X) \rightarrow\left(R^{\prime}, X^{\prime}\right)$ is a morphism in SG then for $A, B \subset X$,

$$
f(A, B) \subset(f(A), f(B)) \cap f(R) .
$$

Let $A \subset R$ be additively closed. A subset $B$ of $A$ is called normal (in $A$ ) if $(A, B) \subset B$. We remark that in [40, p. 24], the terminology " $B$ is an ideal in $A$ " is employed. By (1.14) and (1.17), a normal subset is in particular closed. Moreover, by (1.13) and (1.14), $\varnothing$ and $A$ are always normal subsets of $A$, and by (1.15) any proper normal subset $B$ of $A$ has $0 \notin B^{c}$.

Examples. (a) Let $R$ be symmetric, i.e., $R=-R$, and let $P \subset R$ be closed. As an intersection of closed subsets, the symmetric part $P_{s}:=P \cap(-P)$ of $P$ is closed. Moreover, the unipotent part $P_{u}:=P \backslash(-P)$ of $P$ is normal in $P$, cf. $[26,10.6]$. Other examples of normal subsets will be given in $\S 1.3$ and $§ 1.4$.

(b) Let $(R, X)=(\mathbb{Z}, \mathbb{Z})$. For $a, b \in \mathbb{N}_{+}$, the structure of the root intervals $(a, b)$ and $[a, b]$ is closely tied to the so-called "postage stamp problem" of number theory. It is no great restriction of generality to assume $a$ and $b$ relatively prime. Then it is a well-known exercise in elementary number theory that $a b \notin(a, b)$ while every integer $n>a b$ is contained in $(a, b)$. Obviously, $a+b$ is the smallest element in $(a, b)$, but the precise structure of the gaps between $a+b$ and $a b$ in $(a, b)$ seems to be unknown. Similarly, if $a>1$ and $b>1$ then $a b-(a+b)$ does not belong to $[a, b]$ but every $n>a b-(a+b)$ does.

\subsection{The lower central series}

Let $(R, X) \in \mathbf{S G}$ and let $A \subset R$ be an arbitrary subset. The lower central series of $A$ is defined inductively by

$$
\ell^{1}(A)=A^{c}, \quad \bigodot^{n+1}(A)=\left(A, \bigodot^{n}(A)\right) .
$$


From (1.16) and (1.17) it follows by induction that

$$
\begin{aligned}
& \complement^{n}(A)=\ell^{n}\left(A^{c}\right)=\ell^{n}(A)^{c}, \\
& \complement^{n}(A) \supset \bigodot^{n+1}(A),
\end{aligned}
$$

and (1.15) and (1.13) yield

$$
0 \in A^{c} \quad \Longrightarrow \quad e^{n}(A)=A^{c},
$$

for all $n \geq 1$. Thus the lower central series is mainly of interest for closed subsets not containing 0 , i.e., for strictly positive subsets, cf. (1.4). We note also that all $e^{n}(A)$ are normal subsets of $A$ if $A$ is closed. The lower central series behaves well with respect to inclusions and morphisms:

$$
\begin{aligned}
& B \subset A \Longrightarrow \varphi^{n}(B) \subset \bigodot^{n}(A), \\
& f\left(\ell^{n}(A)\right) \subset \ell^{n}(f(A)) .
\end{aligned}
$$

Indeed, (1.25) is a consequence of (1.17) while (1.26) follows from (1.3) and (1.20).

Remark. Our requirement that $X$ be torsion-free is explained by the following fact. Let $X$ be any abelian group, and define closedness of subsets as in $§ 1.1$. Then a closed subset of $X$ containing a torsion element necessarily contains 0 and hence $\mathcal{C}^{n}(A)=A$ for all $n \in \mathbb{N}_{+}$.

\subsection{The upper central series}

Let $(R, X) \in \mathbf{S G}$ and let $A \subset R$ be a closed subset. We define the upper central series of $A$ inductively by

$$
\mathcal{Z}_{0}(A)=\emptyset, \quad \mathcal{Z}_{n}(A)=\left\{\gamma \in A:(\gamma, A) \subset \mathcal{Z}_{n-1}(A)\right\},
$$

and the centre of $A$ by

$$
\mathcal{Z}(A):=\mathcal{Z}_{1}(A)=\{\gamma \in A:(\gamma, A)=\emptyset\} .
$$

From the definition, it is clear that

$$
\emptyset=Z_{0}(A) \subset \mathfrak{Z}_{1}(A) \subset \mathbb{Z}_{2}(A) \subset \cdots \subset A,
$$

and that

$$
\left(A, \mathcal{Z}_{n}(A)\right) \subset \mathfrak{Z}_{n-1}(A),
$$

in particular, the $\mathcal{Z}_{n}(A)$ are normal in $A$. 
As for the lower central series, only the case $0 \notin A$ is of interest, because $0 \in A$ implies $\gamma=\gamma+0 \in(\gamma, A)$ for all $\gamma \in A$, so $\mathcal{Z}(A)$ and therefore also all the other $\mathrm{Z}_{n}(A)$ are empty.

\subsection{Prenilpotent and nilpotent subsets}

Let $(R, X) \in \mathbf{S G}$. A subset $A$ of $R$ is said to be prenilpotent if $\varphi^{n}(A)=\varnothing$ for sufficiently large $n$, and it is called nilpotent if it is closed and prenilpotent. From the definition of strict positivity and from 1.3 it is immediate that

$$
\begin{aligned}
A \text { nilpotent } & \Longrightarrow A \text { strictly positive, } \\
A \text { prenilpotent } & \Longleftrightarrow A^{c} \text { nilpotent, } \\
A \text { prenilpotent } & \Longrightarrow 0 \notin A^{c}, \\
B \subset A \text { and } A \text { prenilpotent } & \Longrightarrow B \text { prenilpotent } \\
f(A) \text { prenilpotent } & \Longrightarrow A \text { prenilpotent. }
\end{aligned}
$$

The class of a nilpotent $A$ is the smallest $k$ such that $e^{k+1}(A)=\emptyset$. Thus

$$
\begin{aligned}
& k \leq 1 \quad \Longleftrightarrow A A=\mathbb{Z}(A) \quad \Longleftrightarrow \quad(A, A) \subset \mathcal{Z}(A) \quad \Longleftrightarrow \quad(A, A)=\emptyset, \\
& k \leq 2 \quad \Longleftrightarrow(A,(A, A))=\emptyset,
\end{aligned}
$$

and we will call an $A$ of class $\leq 1$ resp. $\leq 2$ abelian resp. 2-step nilpotent.

As in the case of groups, nilpotence can also be characterized by the upper central series. More generally, let $A$ be a strictly positive subset of $R$. A chain of subsets $A \supset A_{1} \supset A_{2} \supset \cdots$ is called a central chain if $\left(A, A_{n}\right) \subset A_{n+1}$ for all $n \geq 1$. For example, the lower central series is a central chain, and so is $A_{i}:=Z_{m+1-i}$ for some fixed $m$, provided we let $\mathcal{Z}_{j}(A)=\emptyset$ for $j<0$.

Clearly the terms $A_{n}$ of a central chain are normal in $A$. From (1.21) and (1.27) it follows easily that

$$
\begin{aligned}
A_{1}=A & \quad \Longrightarrow \quad A_{i} \supset \bigodot^{i}(A), \\
A_{n+1}=\emptyset & \Longrightarrow A_{i} \subset Z_{n+1-i}(A) .
\end{aligned}
$$

Now (1.36) shows

$$
A \text { is nilpotent of class } \leq n \quad \Longleftrightarrow \quad \begin{aligned}
& \text { there exists a central chain } \\
& \text { with } A_{1}=A \text { and } A_{n+1}=\emptyset
\end{aligned}
$$


and (1.37) implies

$A$ is nilpotent of class $\leq n \quad \Longleftrightarrow \quad Z_{n}(A)=A$.

Let us also note that the length of the upper central series of a nilpotent $A$ of class $k$ is exactly $k$. Indeed, $\mathfrak{Z}_{k}(A)=A$ holds by (1.39). Assuming $\mathfrak{Z}_{k-1}(A)=A$ would yield a central chain $A_{i}:=Z_{k-i}(A)$ with $A_{1}=A$ and $A_{k}=\mathfrak{Z}_{0}(A)=\emptyset$, so $A$ would have class $\leq k-1$, contradiction.

Examples 1.2. (a) A nilpotent subset need not be finite (but see Lemma 1.4 and Proposition 1.6). For example, $A=\left\{\varepsilon_{0}-\varepsilon_{i}: i \in \mathbb{N}, i \geq 1\right\}$ is an abelian subset of the root system $R=\dot{\mathrm{A}}_{\mathbb{N}}=\left\{\varepsilon_{i}-\varepsilon_{j}: i, j \in \mathbb{N}\right\}$ in the notation of [26, 8.1].

(b) Let $f:(R, X) \rightarrow(\mathbb{Z}, \mathbb{Z})$ be a morphism in SG. Put $R_{n}=\{\alpha \in R: f(\alpha)=$ $n\}$. Then $A=\bigcup_{k \geq 1} R_{k}$ is a strictly positive subset and $A_{n}:=\bigcup_{k \geq n} R_{k}$ defines a central chain of $A$ which even satisfies $\left(A_{i}, A_{j}\right) \subset A_{i+j}$. If $f(\bar{A})$ is bounded above by some $n \in \mathbb{N}$, then $A$ is nilpotent of class $\leq n$.

For example, if $R$ is a locally finite root system over $\mathbb{R}$, a morphism $f$ is just a coweight of $R$, cf. [26, 7.1,7.5(2)]. In case $f$ is a basic coweight, we have $A_{7}=\emptyset$ by [26, 7.12], so $A$ is nilpotent of class $\leq 6$. If $f$ defines a 3-grading of $A$ in the sense of $[26,17.6]$ then $A_{2}=\emptyset$ so $A=R_{1}$ is abelian.

(c) Let $R=\Delta$ be the set of (real and imaginary) roots of a Kac-Moody algebra $g$, and let $\Delta=\Delta_{+} \cup \Delta_{-}$be the usual decomposition of $\Delta$ into positive and negative roots. The height function ht $: R \rightarrow \mathbb{Z}$, as defined in [23, 1.1], is a morphism. In this case, $A=\Delta_{+}$and the central chain $A_{n}$ defined by the height function is used in [24, 6.1.1] to give $\hat{\mathrm{g}}_{+}=\prod_{\alpha \in \Delta_{+}} \mathrm{g}_{\alpha}$ the structure of a pro-nilpotent Lie algebra.

The following lemma gives a detailed description of the prenilpotent twoelement subsets of locally finite root systems.

Lemma 1.3. Let $(R, X)$ be a locally finite root system, let $\{\alpha, \beta\} \subset R$ be prenilpotent, and put $R_{\alpha \beta}:=R \cap(\mathbb{Z} \alpha+\mathbb{Z} \beta)$ and $C:=(\alpha, \beta)$. Then $[\alpha, \beta]$ is nilpotent of class $k \leq 5$ and of cardinality $\leq 6$. Moreover, Card $C \leq 4$, Card $(C, C) \leq 1$, and $C \neq \varnothing$ if and only if $\alpha+\beta \in R$.

Proof. This follows easily from the classification of root systems of rank $\leq 2$ in [14]. The details are left to the reader. Note that, by (1.18), $[\alpha, \beta]=\{\alpha\}^{c} \cup C \cup$ $\{\beta\}^{c}$ from which it follows easily that

$$
\bigodot^{2}([\alpha, \beta])=\left(\{\alpha\}^{c} \backslash\{\alpha\}\right) \cup C \cup\left(\{\beta\}^{c} \backslash\{\beta\}\right) .
$$


Also, $\{\alpha\}^{c}=\{\alpha, 2 \alpha\}$ or $\{\alpha\}^{c}=\{\alpha\}$ depending on whether $2 \alpha$ does or does not belong to $R$.

We now list the cases where $C \neq \emptyset$ in more detail. It is no restriction to assume that $\|\alpha\| \leq\|\beta\|$ with respect to some invariant inner product.

\begin{tabular}{|c|r|r|c|c|c|c|}
\hline Case & $\left\langle\alpha, \beta^{\vee}\right\rangle$ & $\left\langle\beta, \alpha^{\vee}\right\rangle$ & $C=(\alpha, \beta)$ & $k$ & $\mid[\alpha, \beta]$ & $R_{\alpha \beta}$ \\
\hline 1 & 2 & 2 & $2 \alpha$ & 2 & 2 & $\mathrm{BC}_{1}$ \\
\hline 2 & 1 & 1 & $\alpha+\beta$ & 2 & 3 & $\mathrm{G}_{2}$ \\
\hline 3 & 0 & 0 & $\alpha+\beta$ & 2 & 3 or 5 & $\mathrm{~B}_{2}$ or $\mathrm{BC}_{2}$ \\
\hline 4 & -1 & -1 & $\alpha+\beta$ & 2 & 3 & $\mathrm{~A}_{2}$ \\
\hline 5 & -1 & -1 & $\begin{array}{c}\alpha+\beta, 2 \alpha+\beta, \\
\alpha+2 \beta\end{array}$ & 3 & 5 & $\mathrm{G}_{2}$ \\
\hline 6 & -1 & -2 & $\alpha+\beta, 2 \alpha+\beta$ & 3 & 4 & $\mathrm{~B}_{2}$ \\
\hline 7 & -1 & -2 & $\begin{array}{c}\alpha+\beta, 2 \alpha+\beta, \\
2 \alpha+2 \beta\end{array}$ & 4 & 6 & $\mathrm{BC}_{2}$ \\
\hline 8 & -1 & -3 & $\begin{array}{c}\alpha+\beta, 2 \alpha+\beta, \\
3 \alpha+\beta, 3 \alpha+2 \beta\end{array}$ & 5 & 6 & $\mathrm{G}_{2}$ \\
\hline
\end{tabular}

Remarks. We put $B:=\{\alpha, \beta\}$.

Case 1: Here $\alpha=\beta$.

Case 2: $\alpha$ and $\beta$ are two short roots of $\mathrm{G}_{2}$ whose sum is a long root.

Case 3: $\alpha$ and $\beta$ are weakly orthogonal short roots.

Case 4: $B$ is a root basis of $\mathrm{A}_{2}$.

Case 5: $B$ is a root basis for the subsystem of short roots of $\mathrm{G}_{2}$.

Case 6: $R_{\alpha \beta}=\mathrm{B}_{2}$ and $B$ is a root basis of $\mathrm{B}_{2}$.

Case 7: $B$ is a root basis of $\mathrm{BC}_{2}$.

Case 8: $B$ is a root basis of $\mathrm{G}_{2}$.

Lemma 1.4. Let $(R, X) \in \mathbf{S G}$ and let $A \subset R$ be a strictly positive subset of finite cardinality $n$.

(a) There exist total orders $\geq$ on A compatible with the partial order $\succeq_{A}$ defined by $A$ in the sense that $\alpha \geq_{A} \beta$ implies $\alpha \geq \beta$. 
(b) Let $\geq$ be as in (a), and enumerate $A=\left\{\alpha_{1}, \ldots, \alpha_{n}\right\}$ in such a way that $\alpha_{1}<\cdots<\alpha_{n}$. Then $A_{i}:=\left\{\alpha_{i}, \ldots, \alpha_{n}\right\}$ for $i=1, \ldots, n$, and $A_{i}:=\emptyset$ for $i>n$, is a central chain of $A$. In particular, $A$ is nilpotent of class $\leq n$.

Proof. (a) This follows from the Szpilrajn-Marczewski Lemma [21, Chapter 8, Section 8.6].

(b) We show ( $\left.A, A_{i}\right) \subset A_{i+1}$. By (1.11), an element $\gamma \in\left(A, A_{i}\right)$ has the form $\gamma=\alpha_{i_{1}}+\cdots+\alpha_{i_{p}}+\alpha_{j_{1}}+\cdots+\alpha_{j_{q}}$ where $p, q \geq 1, i_{\lambda} \in\{1, \ldots, n\}$ and $j_{\mu} \in\{i, \ldots, n\}$; in particular, $\gamma \succ_{A} \alpha_{j_{1}}$. On the other hand, $\gamma \in A$ because $A$ is closed, say, $\gamma=\alpha_{k}$. Hence $k>j_{1} \geq i$ so $\alpha_{k} \in A_{k} \subset A_{i+1}$.

The statement about the nilpotence of $A$ now follows from (1.38).

Lemma 1.5. Let $F$ be a finite set and let $\mathbb{N} F$, the set of functions $F \rightarrow \mathbb{N}$, be equipped with the partial order

$$
v \leq w \quad \Longleftrightarrow \quad v(\alpha) \leq w(\alpha) \text { for all } \alpha \in F .
$$

Then every infinite subset $S$ of $\mathbb{N}^{F}$ contains a strictly increasing sequence $v_{1}<$ $v_{2}<\cdots$.

Proof. The proof is by induction on the cardinality of $F$, the case $F=\emptyset$ being trivial. If $S$ has no maximal element then the assertion is clear. Otherwise, let $m$ be a maximal element of $S$. Then $v>m$ holds for no $v \in S$, i.e., for every $v \in S$ there exists an element $\alpha \in F$ such that $v(\alpha) \leq m(\alpha)$. Letting $S_{\alpha}:=\{v \in S$ : $v(\alpha) \leq m(\alpha)\}$, we thus have $S=\bigcup_{\alpha \in F} S_{\alpha}$. Since $S$ is infinite, there must be a $\beta \in F$ such that $S_{\beta}$ is infinite. Consider the evaluation map $S_{\beta} \rightarrow \mathbb{N}, v \mapsto v(\beta)$, whose image is contained in the finite interval $I:=\{0,1, \ldots, m(\beta)\}$ of $\mathbb{N}$. Since $S_{\beta}$ is infinite, there exists $i \in I$ such that the fibre $S_{\beta}^{i}:=\left\{v \in S_{\beta}: v(\beta)=i\right\}$ is infinite. Let $F^{\prime}:=F \backslash\{\beta\}$, denote by res $: \mathbb{N}^{F} \rightarrow \mathbb{N}^{F^{\prime}}$ the restriction map induced by the inclusion $F^{\prime} \hookrightarrow F$, and put $S^{\prime}:=\operatorname{res}\left(S_{\beta}^{i}\right) \subset \mathbb{N}^{F^{\prime}}$. Clearly, res : $S_{\beta}^{i} \rightarrow S^{\prime}$ is bijective, with inverse ext $: S^{\prime} \rightarrow S_{\beta}^{i}$ given by extending an element $v^{\prime} \in S^{\prime}$ (which after all is a map $F^{\prime} \rightarrow \mathbb{N}$ ) to a map $F \rightarrow \mathbb{N}$ via $\beta \mapsto i$. By induction, there exists a strictly increasing sequence $v_{1}^{\prime}<v_{2}^{\prime}<\cdots$ in $S^{\prime}$. Then $v_{k}:=\operatorname{ext}\left(v_{k}^{\prime}\right)$ is the desired sequence in $S$.

Proposition 1.6. Let $(R, X) \in$ SG. For a subset $F \subset R$, the following conditions are equivalent:

(i) $F$ is finite and prenilpotent,

(ii) $F^{c}$ is finite and $0 \notin F^{c}$,

(iii) $F^{c}$ is finite and nilpotent,

(iv) $F^{c}$ is finite and strictly positive. 
Proof. (i) $\Longrightarrow$ (ii): $0 \notin F^{c}$ holds by (1.33). Now assume, by way of contradiction, that $F^{c}$ is infinite. Then by definition of the closure of a set in (1.1) we have $S:=\kappa^{-1}\left(F^{c}\right) \subset \mathbb{N}^{F}$ infinite. Choose a sequence $\left(v_{k}\right)_{k \geq 1}$ in $S$ as in Lemma 1.5 and put $\gamma_{k}=\kappa\left(v_{k}\right)$. We will show by induction that $\gamma_{k} \in \mathcal{C}^{k}(F)$ for all $k \geq 1$, contradicting the fact that $\ell^{k}(F)=\emptyset$ for sufficiently big $k$, by nilpotence of $F^{c}$. Obviously, $\gamma_{1} \in F^{c}=\mathcal{C}^{1}(F)$. Suppose we have $\gamma_{k} \in \mathcal{C}^{k}(F)$. Then $\gamma_{k+1}-\gamma_{k}=$ $\sum_{\alpha \in F} n_{\alpha} \alpha$ where all $n_{\alpha}:=v_{k+1}(\alpha)-v_{k}(\alpha) \in \mathbb{N}$, and at least one $n_{\alpha}$ is positive because $v_{k+1}>v_{k}$. Hence $\gamma_{k+1} \in\left(F, \gamma_{k}\right) \subset\left(F, \ell^{k}(F)\right)=e^{k+1}(F)$.

(ii) $\Longrightarrow$ (i) follows immediately from Lemma 1.4(b). The equivalence of (i), (ii) and (iii) now follows from (1.32), and that of (ii) and (iv) from (1.4).

\section{Reflection systems}

\subsection{Pre-reflection systems}

From now on $\mathbb{K}$ denotes a field of characteristic zero. We introduce a subcategory of the category $\mathbf{S} \mathbf{V}_{\mathbb{K}}[26,1.1]$. Recall that the objects of $\mathbf{S V}_{\mathbb{K}}$ are the pairs $(R, X)$, where $X$ is a $\mathbb{K}$-vector space and $R \subset X$ is a subset of $X$ which spans $X$ and contains the zero vector of $X$. A morphism $f:(R, X) \rightarrow(S, Y)$ in $\mathbf{S V}_{\mathbb{K}}$ is a $\mathbb{K}$-linear map $f: X \rightarrow Y$ such that $f(R) \subset S$.

Let $X$ be a vector space over $\mathbb{K}$. By a (hyperplane) reflection we mean an element $\sigma$ of $\operatorname{GL}(X)$ with $\sigma^{2}=$ Id and fixed point set a hyperplane. Thus, a reflection $\sigma$ is uniquely determined by the hyperplane $\operatorname{Ker}(\operatorname{Id}-\sigma)$ and the line $\operatorname{Ker}(\operatorname{Id}+\sigma)$. We denote by $\operatorname{Ref}(X)$ the union of $\left\{\operatorname{Id}_{X}\right\}$ and all hyperplane reflections of $X$, thus considering $\operatorname{Id}_{X}$ as an improper reflection.

Now let $(R, X) \in \mathbf{S V}_{\mathbb{K}}$, and let $s: R \rightarrow \operatorname{Ref}(X)$ be a map, written $\alpha \mapsto s_{\alpha}$. We put

$$
R^{\mathrm{im}}:=\left\{\alpha \in R: s_{\alpha}=\mathrm{Id}\right\}, \quad R^{\mathrm{re}}=R \backslash R^{\mathrm{im}},
$$

called the imaginary roots and reflective roots, respectively. This terminology is of course suggested by the example of root systems of Kac-Moody Lie algebras where the reflective roots are just the real roots, cf. $\$ 2.12$ (c). The triple $(R, X, s)$ is called a pre-reflection system if the following axioms hold for all $\alpha \in R$ :

$(\operatorname{ReS} 1) \alpha \in R^{\text {re }}$ implies $\alpha \neq s_{\alpha}(\alpha)=-\alpha \in R^{\text {re }}$;

$(\operatorname{ReS} 2) s_{\alpha}\left(R^{\mathrm{re}}\right)=R^{\mathrm{re}}$ and $s_{\alpha}\left(R^{\mathrm{im}}\right)=R^{\mathrm{im}}$.

Note that (ReS1) and (ReS2) imply

$$
0 \in R^{\mathrm{im}}, \quad R^{\mathrm{re}}=-R^{\mathrm{re}} \quad \text { and } \quad s_{\alpha}(R)=R
$$


for all $\alpha \in R$. The subgroup of $\operatorname{GL}(X)$ generated by all $s_{\alpha}, \alpha \in R$, is called the Weyl group of $(R, X, s)$ and denoted by $W(R, X, s)$ or simply $W(R)$ if $(R, X, s)$ is clear from the context.

Let $(S, Y, s)$ be a second pre-reflection system. Unless this might lead to confusion, we will use the same letter $s$ for the maps $R \rightarrow \operatorname{Ref}(X)$ and $S \rightarrow \operatorname{Ref}(Y)$. A morphism $f:(R, X, s) \rightarrow(S, Y, s)$ is a linear map $f: X \rightarrow Y$ such that $f(R) \subset S$ and

$$
f\left(s_{\alpha}(\beta)\right)=s_{f(\alpha)}(f(\beta)),
$$

for all $\alpha, \beta \in R$. With these definitions, pre-reflection systems form a category which shall remain nameless. As usual, the automorphism group of $(R, X, s)$ is denoted by $\operatorname{Aut}(R, X, s)$ or simply by $\operatorname{Aut}(R)$. Note that $(-R, X)$ together with the maps $s_{-\alpha}=s_{\alpha}$ for $\alpha \in R$ is a pre-reflection system isomorphic to $(R, X, s)$ via - Id.

As $R$ spans $X$, formula (2.3) for a morphism $f$ holds with $\beta$ replaced by an arbitrary $x \in X$, i.e.,

$$
f \circ s_{\alpha}=s_{f(\alpha)} \circ f \text {. }
$$

Also observe that for any morphism $f:(R, X, s) \rightarrow(S, Y, s)$,

$$
f\left(R^{\mathrm{im}}\right) \subset S^{\mathrm{im}}, \quad f\left(R^{\mathrm{re}}\right) \subset S^{\mathrm{re}} \cup\{0\} .
$$

Indeed, if $s_{\alpha} \neq \operatorname{Id}_{X}$ but $s_{f(\alpha)}=\operatorname{Id}_{Y}$ then

$$
-f(\alpha)=f(-\alpha)=f\left(s_{\alpha}(\alpha)\right)=s_{f(\alpha)}(f(\alpha))=f(\alpha)
$$

shows $2 f(\alpha)=0$. Likewise, $s_{\alpha}=\operatorname{Id}$ implies $f(\alpha)=f\left(s_{\alpha}(\alpha)\right)=s_{f(\alpha)} f(\alpha)$ and hence $s_{f(\alpha)}=$ Id. Thus, (2.5) holds. In fact, if $f:(R, X) \rightarrow(S, Y)$ is a morphism in $\mathbf{S} \mathbf{V}_{\mathbb{K}}$, it is easily seen that

$f$ is a morphism of pre-reflection systems

$$
\Longleftrightarrow \quad f\left(R^{\mathrm{im}}\right) \subset S^{\mathrm{im}} \text { and } f \circ s_{\alpha}=s_{f(\alpha)} \circ f \text { for all } \alpha \in R^{\mathrm{re}} .
$$

\subsection{Reflection systems}

A pre-reflection system $(R, X, s)$ is called a reflection system if it satisfies the following two additional axioms:

(ReS3) $s_{c \alpha}=s_{\alpha}$ whenever $c \in \mathbb{K}^{\times}$and both $\alpha$ and $c \alpha$ belong to $R^{\text {re }}$, and

(ReS4) $s_{\alpha} \in \operatorname{Aut}(R)$, for all $\alpha \in R$,

equivalently, because of (ReS1) and $s_{\alpha}=s_{\alpha}^{-1}$,

$(\operatorname{ReS} 4)^{\prime} s_{s_{\alpha}(\beta)}=s_{\alpha} s_{\beta} s_{\alpha}$ for all $\alpha, \beta \in R$. 
Morphisms between reflection systems are defined to be morphisms of the underlying pre-reflection systems. We denote by $\operatorname{ReS}$ the category of reflection systems, which is thus a full subcategory of the category of pre-reflection systems. By abuse of notation, we will often refer to a reflection system simply by $R$ instead of $(R, X, s)$.

Since the reflections $s_{\alpha}$ are automorphisms of $R$, it would be natural to call $W(R)$ the inner automorphism group of $R$. But following tradition, we will retain the name Weyl group.

It is immediate from (2.4) that $W(R)$ is a normal subgroup of $\operatorname{Aut}(R)$.

Example. We leave it to the reader to show that a pre-reflection system $(R, X, s)$ with $\operatorname{dim} X=1$ is necessarily a reflection system. This is no longer true in higher dimensions, as the following example shows.

Let $X=\mathbb{K}^{2}$ with standard basis $e_{1}, e_{2}$ and let $G$ be the subgroup of $\mathrm{GL}_{2}(\mathbb{K})$ generated by $A=\left(\begin{array}{cc}-1 & 0 \\ 0 & 1\end{array}\right)$ and $B=\left(\begin{array}{cc}-1 & 2 \\ 0 & 1\end{array}\right)$. Note that $A^{2}=B^{2}=$ Id but $A B=\left(\begin{array}{cc}1 & -2 \\ 0 & 1\end{array}\right)$ has infinite order. It follows from $A\left(e_{1}\right)=B\left(e_{1}\right)=-e_{1}$ that $g\left(e_{1}\right)= \pm e_{1}$ for every $g \in G$. Hence $g\left(e_{2}\right) \notin \mathbb{K} \cdot e_{1}$, otherwise $g\left(e_{2}\right)=\lambda e_{1}$ would imply $g^{-1}\left(e_{1}\right)=\lambda^{-1} e_{2} \neq \pm e_{1}$. Now define

$$
R^{\mathrm{re}}=\left\{ \pm e_{1}, \pm 2 e_{1}\right\}, \quad R^{\mathrm{im}}=\{0\} \cup\left\{g\left(e_{2}\right): g \in G\right\}, \quad R=R^{\mathrm{re}} \cup R^{\mathrm{im}} .
$$

Then $R$ is the disjoint union of $R^{\mathrm{re}}$ and $R^{\mathrm{im}}$. Define the reflections $s_{\alpha}$ by

$$
s_{\alpha}= \begin{cases}\text { Id } & \text { for } \alpha \in R^{\text {im }}, \\ A & \text { for } \alpha \in\left\{e_{1},-e_{1}\right\}, \\ B & \text { for } \alpha \in\left\{2 e_{1},-2 e_{1}\right\} .\end{cases}
$$

One sees immediately that the axioms $(\operatorname{ReS} 1)$ and $(\operatorname{ReS} 2)$ hold so $(R, X, s)$ is a pre-reflection system. On the other hand, (ReS3) does not hold because for $\alpha=e_{1}$ and $\beta=2 e_{1}$ we have $s_{\alpha}=A \neq B=s_{\beta}$. Likewise, (ReS4) fails because $s_{\alpha}(\beta)=-\beta$, so $s_{s_{\alpha} \beta}=s_{-\beta}=B$ while $s_{\alpha} s_{\beta} s_{\alpha}=A B A \neq B$.

\subsection{The map $\alpha \mapsto \alpha^{\vee}$}

Let $(R, X, s)$ be a pre-reflection system. For every reflective root $\alpha$, there exists a unique linear form $\alpha^{\vee}$ on $X$ such that $s_{\alpha}$ is given by the familiar formula

$$
s_{\alpha}(x)=x-\left\langle x, \alpha^{\vee}\right\rangle \alpha .
$$

In particular, $s_{\alpha}(\alpha)=-\alpha \Longleftrightarrow\left\langle\alpha, \alpha^{\vee}\right\rangle=2$. For $\alpha \in R^{\text {im }}$ we put $\alpha^{\vee}=0$. Then $\checkmark: R \rightarrow X^{*}$ is a well-defined map and (2.7) holds for all $\alpha \in R$ and $x \in X$.

Conversely, given $(R, X) \in \mathbf{S V}_{\mathbb{K}}$ with a map ${ }^{\vee}: R \rightarrow X^{*}$, taking (2.7) as the definition of $s_{\alpha}$ and putting $R^{\mathrm{re}}=\left\{\alpha \in R: \alpha^{\vee} \neq 0\right\}$ and $R^{\mathrm{im}}=R \backslash R^{\mathrm{re}}$, 
the axioms of a pre-reflection system can also be phrased in terms of $\left(R, X,^{\vee}\right)$ as follows: For all $\alpha \in R$,

$(\operatorname{ReS} 1)^{\vee} \alpha^{\vee} \neq 0$ implies $\left\langle\alpha, \alpha^{\vee}\right\rangle=2$ and $(-\alpha)^{\vee} \neq 0$,

$(\operatorname{ReS} 2)^{\vee} s_{\alpha}(R) \subset R$ and $\left(s_{\alpha} \beta\right)^{\vee}=0 \Longleftrightarrow \beta^{\vee}=0$.

The morphism condition (2.4) is now expressed by

$$
\begin{aligned}
f \circ s_{\alpha}=s_{f(\alpha)} \circ f & \Longleftrightarrow\left\langle x, \alpha^{\vee}\right\rangle f(\alpha)=\left\langle f(x), f(\alpha)^{\vee}\right\rangle f(\alpha) \text { for all } x \in X \\
& \Longleftrightarrow f(\alpha)=0 \text { or } f^{*}\left(f(\alpha)^{\vee}\right)=\alpha^{\vee} .
\end{aligned}
$$

Here $f^{*}: Y^{*} \rightarrow X^{*}$ is the map sending a linear form $h$ on $Y$ to the linear form $h \circ f$ on $X$. Suppose $f$ is a vector space isomorphism, and define $f^{\vee}: X^{*} \rightarrow Y^{*}$ by $f^{\vee}:=\left(f^{*}\right)^{-1}$. Then (2.8) shows that $f$ is an isomorphism of pre-reflection systems if and only if $f(R)=S$ and

$$
f(\alpha)^{\vee}=f^{\vee}\left(\alpha^{\vee}\right)
$$

for all $\alpha \in R$. We can also re-formulate the axioms (ReS3) and (ReS4) of a reflection system in terms of the map $\alpha \mapsto \alpha^{\vee}$, namely:

$(\operatorname{ReS} 3)^{\vee}(c \alpha)^{\vee}=c^{-1} \alpha^{\vee}$ whenever $c \in \mathbb{K}^{\times}$and both $\alpha$ and $c \alpha$ belong to $R^{\text {re }}$, and $(\operatorname{ReS} 4)^{\vee}\left(s_{\alpha} \beta\right)^{\vee}=\beta^{\vee}-\left\langle\alpha, \beta^{\vee}\right\rangle \alpha^{\vee}$ for all $\alpha, \beta \in R$.

Indeed, because $s_{\alpha}=s_{\alpha}^{-1}$ we have

$$
\left\langle x, s_{\alpha}^{\vee}\left(\beta^{\vee}\right)\right\rangle=\left\langle s_{\alpha}(x), \beta^{\vee}\right\rangle=\left\langle x-\left\langle x, \alpha^{\vee}\right\rangle \alpha, \beta^{\vee}\right\rangle=\left\langle x, \beta^{\vee}-\left\langle\alpha, \beta^{\vee}\right\rangle \alpha^{\vee}\right\rangle
$$

for all $x \in X$, whence always $s_{\alpha}^{\vee}\left(\beta^{\vee}\right)=\beta^{\vee}-\left\langle\alpha, \beta^{\vee}\right\rangle \alpha^{\vee}$. Therefore, by (2.9), $s_{\alpha}$ is an automorphism if and only if (ReS4) $)^{\vee}$ holds.

\subsection{Elementary properties}

Let $(R, X, s)$ be a pre-reflection system. We will say $R$ is

(i) reduced if $\alpha \in R^{\mathrm{re}}, c \in \mathbb{K}^{\times}$and $c \alpha \in R^{\mathrm{re}}$ imply $c= \pm 1$ (note that we do not require this condition for roots in $R^{\mathrm{im}}$ );

(ii) saturated if $\mathbb{K}^{\times} R^{\text {re }} \cap R=R^{\text {re }}$, i.e., all roots that are non-zero scalar multiples of reflective roots are themselves reflective;

(iii) integral if $\left\langle R, R^{\vee}\right\rangle \subset \mathbb{Z}$;

(iv) nondegenerate if $\bigcap_{\alpha \in R} \operatorname{Ker}\left(\alpha^{\vee}\right)=\{0\}$;

(v) symmetric if $R=-R$ (cf. §1.2), equivalently, $R^{\mathrm{im}}=-R^{\mathrm{im}}$;

(vi) coherent if for $\alpha, \beta \in R^{\text {re }}$ we have $\left\langle\alpha, \beta^{\vee}\right\rangle=0 \Longleftrightarrow\left\langle\beta, \alpha^{\vee}\right\rangle=0$. 
It is easy to see from $(\mathrm{ReS} 3)^{\vee}$ that in an integral reflection system,

$$
\alpha \in R^{\text {re }} \text { and } c \alpha \in R^{\text {re }} \text { for some } c \in \mathbb{K}^{\times} \quad \Longrightarrow \quad c \in\{ \pm 1 / 2, \pm 1, \pm 2\}
$$

and that $\alpha / 2$ and $2 \alpha$ cannot both be in $R^{\text {re }}$.

For a reflection system $(R, X, s)$ and $\alpha, \beta \in R^{\text {re }}$ we have

$$
s_{\alpha}=s_{\beta} \quad \Longleftrightarrow \quad \beta \in \mathbb{K}^{\times} \alpha .
$$

Indeed, $s_{\alpha}=s_{\beta}$ implies $-\alpha=s_{\beta}(\alpha)=\alpha-\left\langle\alpha, \beta^{\vee}\right\rangle \beta$, whence $\left\langle\alpha, \beta^{\vee}\right\rangle \beta=2 \alpha$ and so $\beta \in \mathbb{K}^{\times} \alpha$. The converse follows from (ReS3). Moreover, a reflection system is automatically coherent. Namely, for a reflection system $R$ and $\alpha, \beta \in R^{\text {re }}$, one knows ([25, 2.2], [28, Chapter 5.2, Proposition 8]) that

$$
\begin{aligned}
\left\langle\alpha, \beta^{\vee}\right\rangle=0 \quad & \Longleftrightarrow \quad s_{\alpha} s_{\beta}=s_{\beta} s_{\alpha} \text { and } \alpha \notin \mathbb{K}^{\times} \beta \\
& \Longleftrightarrow\left\langle\beta, \alpha^{\vee}\right\rangle=0 .
\end{aligned}
$$

For the convenience of the reader, we indicate a proof. As the condition in the middle is symmetric in $\alpha$ and $\beta$, it suffices to prove the first equivalence. To do so, observe that $\left\langle\alpha, \beta^{\vee}\right\rangle=0$ obviously implies $\alpha \notin \mathbb{K}^{\times} \beta$ and $s_{\beta}(\alpha)=\alpha$, whence $s_{\beta} s_{\alpha}=s_{\alpha} s_{\beta}$ by $(\operatorname{ReS} 4)^{\prime}$. Conversely, $s_{\beta} s_{\alpha}=s_{\alpha} s_{\beta}$ together with (ReS4) yields $s_{\beta}=s_{\gamma}$ for $\gamma=s_{\alpha}(\beta)$, hence $\gamma=\beta-\left\langle\beta, \alpha^{\vee}\right\rangle \alpha \in \mathbb{K}^{\times} \beta$ by $\S(2.11)$ and thus $\left\langle\beta, \alpha^{\vee}\right\rangle \alpha \in \mathbb{K} \beta$. But then $\left\langle\beta, \alpha^{\vee}\right\rangle=0$ because of our assumption $\alpha \notin \mathbb{K}^{\times} \beta$.

Let $\left(R_{i}, X_{i}, s_{i}\right)_{i \in I}$ be a family of pre-reflection systems, and let

$$
(R, X)=\coprod_{i \in I}\left(R_{i}, X_{i}\right)=\left(\bigcup_{i \in I} R_{i}, \bigoplus_{i \in I} X_{i}\right)
$$

be its coproduct in the category $\mathbf{S V}_{\mathbb{K}}$, cf. [26, 1.2]. We extend each $s_{\alpha_{i}}, \alpha_{i} \in R_{i}$, to a reflection on $X$ by $s_{\alpha_{i}} \mid X_{j}=$ Id for $i \neq j$, and in this way obtain a map $s: R \rightarrow \operatorname{Ref}(X)$ which is easily seen to satisfy $(\operatorname{ReS} 1)$ and $(\operatorname{ReS} 2)$. The linear form on $X$ corresponding to $\alpha_{i} \in R_{i}$ is just the extension by zero of $\alpha_{i}^{\vee}$. By abuse of notation we will also write $R=\bigoplus_{i \in I} R_{i}$, and call $R$ the direct sum of the pre-reflection systems $\left(R_{i}, X_{i}, s_{i}\right)_{i \in I}$. It is immediate that $R^{\mathrm{re}}=\bigcup_{i \in I} R_{i}^{\mathrm{re}}$ and $R^{\mathrm{im}}=\bigcup_{i \in I} R_{i}^{\mathrm{im}}$. Moreover, $R$ is a reflection system if and only if each $R_{i}$ is a reflection system, and in this case $W(R) \cong \bigoplus_{i \in I} W\left(R_{i}\right)$, the restricted direct product of the family of Weyl groups $\left(W\left(R_{i}\right)\right)_{i \in I}$. Similarly, $R$ has any one of the properties (i)-(vi) defined above if and only if each $R_{i}$ does.

A pre-reflection system with a non-empty set of real roots is called indecomposable if it is not isomorphic to a direct sum of two pre-reflection systems, each of which has a non-empty set of real roots. 


\subsection{Subsystems}

Let $(R, X, s)$ be a pre-reflection system. By a subsystem of $(R, X, s)$ we mean a pre-reflection system $\left(R^{\prime}, X^{\prime}, s^{\prime}\right)$ where $R^{\prime} \subset R$ (and hence in particular $X^{\prime}=$ $\operatorname{Span}\left(R^{\prime}\right)$ is a subspace of $X$ ), and the inclusion $X^{\prime} \hookrightarrow X$ is a morphism. This just means that $s_{\alpha}^{\prime}(\beta)=s_{\alpha}(\beta)$ for all $\alpha, \beta \in R^{\prime}$. It is immediately seen that subsystems are in natural bijection with subsets $R^{\prime}$ of $R$ satisfying $0 \in R^{\prime}$ and $s_{\alpha}(\beta) \in R^{\prime}$ for all $\alpha, \beta \in R^{\prime}$. This bijection will be treated as an identification in the sequel.

Any subsystem $R^{\prime}$ of $R$ gives rise to the subgroup $W_{R^{\prime}}(R)$ of $W(R)$ generated by all $s_{\alpha}, \alpha \in R^{\prime}$. The restriction map res : $W_{R^{\prime}}(R) \rightarrow W\left(R^{\prime}\right), w \mapsto w \mid X^{\prime}$, is a surjective homomorphism which need not be injective (see, however, $[26,5.8]$ as well as the remark in Lemma 2.4 and Lemma 4.1). We now give some examples of subsystems.

(a) It follows from $(\operatorname{ReS} 2)$ that

$$
\operatorname{Re}(R):=R^{\mathrm{re}} \cup\{0\}
$$

is always a subsystem, and from (2.5) that the assignment $\operatorname{Re}: R \mapsto \operatorname{Re}(R)$ is an idempotent functor from the category of reflection systems to itself. Similarly,

$$
R_{\text {ind }}:=\left\{\alpha \in R^{\text {re }}: \alpha / 2 \notin R^{\text {re }}\right\} \cup\{0\},
$$

is a subsystem. We call its elements the indivisible roots. By (2.10), $R_{\text {ind }}$ is a reduced subsystem of any integral reflection system $R$.

(b) Let $k \subset \mathbb{K}$ be a subring containing $\left\langle R, R^{\vee}\right\rangle$ and let $M \subset X$ be a $k$-submodule. Then (2.7) shows that $M \cap R$ is a subsystem. If $k=\mathbb{K}$ such a subsystem will be called full.

(c) Let $E \subset R$ be an arbitrary subset. The subsystem generated by $E$ is the smallest subsystem $S$ containing $E$. If $R$ is a reflection system, $S$ can be described as

$$
S=(H \cdot E) \cup\{0\}
$$

where $H$ is the subgroup of $W(R)$ generated by all $s_{\alpha}, \alpha \in E$.

\subsection{Connectedness, cf. [26, 3.12]}

Let $(R, X, s)$ be a coherent pre-reflection system, and let $A$ be a subset of $\operatorname{Re}(R)$ with $0 \in A$. Two roots $\alpha$ and $\beta$ of $A^{\times}$are said to be connected in $A$, if there exist finitely many roots $\alpha=\alpha_{0}, \alpha_{1}, \ldots, \alpha_{n}=\beta, \alpha_{i} \in A^{\times}$, such that $\left\langle\alpha_{i}, \alpha_{i-1}^{\vee}\right\rangle \neq 0$ for $i=1, \ldots, n$. We then call $\alpha_{0}, \ldots, \alpha_{n}$ a chain connecting $\alpha$ and $\beta$ in $A$. 
Since $R$ is coherent, connectedness is an equivalence relation on $A^{\times}$. A connected component of $A$ is defined as the union of $\{0\}$ and an equivalence class of $A^{\times}$, and $A$ is called connected if $A$ has only one connected component. In particular we will use this terminology for $\operatorname{Re}(R)$.

As an example, we observe that $\alpha$ and $w \alpha$ are connected for any $\alpha \in R^{\text {re }}$ and $w \in W(R)$. Hence $W(R)$ preserves connected components of $\operatorname{Re}(R)$. It is also easily seen, cf. the proof of [26,3.13], that each connected component $S$ of $\operatorname{Re}(R)$ is a subsystem. Moreover, if $\operatorname{Re}(R)$ is connected then $R$ is indecomposable. The converse need however not be true.

Lemma 2.1. Let $(R, X, s)$ be a nondegenerate coherent pre-reflection system. Then the subsystem $\operatorname{Re}(R)$ is the direct sum of its connected components. In particular, $\operatorname{Re}(R)$ is connected if and only if it is indecomposable.

Proof. The proof is a straightforward generalization of the proof of the corresponding result for locally finite root systems $[26,3.13]$ and is left to the reader.

\subsection{Invariant bilinear forms}

Let $(R, X, s)$ be a pre-reflection system. A symmetric bilinear form $b: X \times X \rightarrow$ $\mathbb{K}$ is called invariant if $b(w x, w y)=b(x, y)$ for all $w \in W(R)$ and $x, y \in X$. Since $W(R)$ is generated by the reflections $s_{\alpha}, \alpha \in R^{\text {re }}$, the invariance under the Weyl group is equivalent to $b\left(s_{\alpha} x, y\right)=b\left(x, s_{\alpha} y\right)$ for all $\alpha \in R^{\text {re }}$ and $x, y \in X$. An easy computation, using the fact that $\left\langle\alpha, \alpha^{\vee}\right\rangle=2$ (because $\alpha \in R^{\text {re }}$ ), shows that this is, in turn, equivalent to

$$
2 b(x, \alpha)=\left\langle x, \alpha^{\vee}\right\rangle b(\alpha, \alpha) \text { for all } x \in X \text { and } \alpha \in R^{\mathrm{re}} .
$$

We will say that $b$ is strictly invariant if (2.15) holds not only for all $\alpha \in R^{\text {re }}$ but for all $\alpha \in R$; thus,

$b$ is strictly invariant

$$
\Longleftrightarrow \quad 2 b(x, \alpha)=\left\langle x, \alpha^{\vee}\right\rangle b(\alpha, \alpha) \quad \text { for all } x \in X \text { and } \alpha \in R .
$$

Obviously, the notions of invariant and strictly invariant form agree if $R$ has no imaginary roots $\neq 0$, i.e., if $R=\operatorname{Re}(R)$.

Let $\operatorname{Rad} b=\{x \in X: b(x, X)=0\}$ be the radical of $b$. Since $\alpha \in R^{\mathrm{im}}$ if and only if $\alpha^{\vee}=0$, it is clear that

$b$ is strictly invariant $\quad \Longleftrightarrow \quad b$ is invariant and $R^{\mathrm{im}} \subset \operatorname{Rad} b$. 
In particular, for any strictly invariant form $b$,

$$
R \cap \operatorname{Rad} b=\{\alpha \in R: b(\alpha, \alpha)=0\} \quad \text { and } \quad \bigcap_{\alpha \in R} \operatorname{Ker}\left(\alpha^{\vee}\right) \subset \operatorname{Rad} b .
$$

Hence a pre-reflection system with a nondegenerate strictly invariant form is nondegenerate in the sense of $\$ 2.4$ (iv). We denote by $d(R)$ the $\mathbb{K}$-vector space of strictly invariant forms on $X$. If $R=\bigoplus_{i \in I} R_{i}$ is a direct sum of pre-reflection systems $\left(R_{i}, X_{i}, s_{i}\right)$, cf. $\S 2.4$, there is a canonical isomorphism given by restriction

$$
d\left(\bigoplus R_{i}\right) \cong \prod d\left(R_{i}\right)
$$

Several criteria for the existence of invariant bilinear forms on pre-reflection systems are given in [25]. For example, by [25, Theorem 1.5], there exists an invariant symmetric bilinear form $b$ satisfying $s_{\alpha} \mid \operatorname{Rad} b=\operatorname{Id}$ for all $\alpha \in R^{\text {re }}$ if and only if the following two conditions are fulfilled:

(i) there exists a family $\left(d_{\alpha}: \alpha \in R^{\text {re }}\right)$ of scalars $d_{\alpha} \in \mathbb{K} \backslash\{0\}$ such that $d_{\alpha}\left\langle\beta, \alpha^{\vee}\right\rangle=d_{\beta}\left\langle\alpha, \beta^{\vee}\right\rangle$ holds for all $\alpha, \beta \in R^{\mathrm{re}}$, and

(ii) there exists a linear map $\Phi: \operatorname{Span}_{\mathbb{K}}\left(R^{\mathrm{re}}\right) \rightarrow X^{*}$ satisfying $\Phi(\alpha)=d_{\alpha} \alpha^{\vee}$ for all $\alpha \in R^{\text {re }}$.

If $b$ exists, one can take $d_{\alpha}=b(\alpha, \alpha)$. Conversely, if (i) and (ii) hold, one defines $b$ on $\operatorname{Span}_{\mathbb{K}}\left(R^{\mathrm{re}}\right) \times X$ by $b(x, y)=\Phi(x)(y)$ and then arbitrarily extends $b$ to all of $X \times X$.

Example. Suppose $W(R)$ is finite, and let $\sigma$ be any bilinear form. Then

$$
b(x, y)=\sum_{w \in W(R)} \sigma(w(x), w(y))
$$

defines an invariant form on $X$. If $R^{\mathrm{im}} \subset \operatorname{Rad} \sigma$ then $b$ is strictly invariant. Other examples and constructions of (strictly) invariant forms are given in \$2.8, Proposition 2.2, §2.9, Lemma 4.1 (e) and in $§ 5$.

In the remainder of this section we will discuss examples of (pre-)reflection systems. More examples are given in $§ 3.1$.

\subsection{Locally finite root systems}

We will use the term "locally finite root system" for a locally finite root system over $\mathbb{K}$ in the sense of [26, 4.14]. As already mentioned in [26, 4.14], the classification of locally finite root systems over $\mathbb{K}$ and over $\mathbb{R}$ is the same.

A pre-reflection system $R$ is a finite root system in the usual sense of [14] (except that we require $0 \in R$ ) resp. a locally finite root system in the sense 
of [26] if and only if $R$ is finite resp. locally finite, integral, and $R^{\text {re }}=R^{\times}$. Indeed, it suffices to remark that the axioms $(\operatorname{ReS} 3)^{\vee}$ and $(\operatorname{ReS} 4)^{\vee}$ of $\$ 2.2$ hold in locally finite root systems; they are, respectively, the formulas (2) of [26, 4.8] and (2) of $[26,3.9]$.

Every locally finite root system $R$ has a nondegenerate strictly invariant form, see Proposition 2.2. Moreover, $R$ is the direct sum of its connected components, see [26, 3.13] or Lemma 2.1. In particular, $R$ is connected if and only if $R$ is indecomposable, in which case $R$ is traditionally called irreducible. Finally, we note that $W(R)$ is in general not a Coxeter group [26, Corollary 9.9].

Proposition 2.2. For an integral pre-reflection system $(R, X, s)$ the following conditions are equivalent:

(i) $R$ is a locally finite root system;

(ii) there exists a nondegenerate strictly invariant form on $(R, X)$, and for every $\alpha \in R$ the set $\left\langle R, \alpha^{\vee}\right\rangle$ is bounded as a subset of $\mathbb{Z}$.

In this case, $(R, X)$ has a unique invariant form $(\mid)$ which is normalized in the sense that for every connected component $C \neq\{0\}$ of $R$

$$
2 \in\left\{(\alpha \mid \alpha): \alpha \in C^{\times}\right\} \subset\{2,4,6,8\} .
$$

In fact, the normalized invariant form ( | ) satisfies

$$
\left\{(\alpha \mid \alpha): \alpha \in C^{\times}\right\} \in\{\{2\},\{2,4\},\{2,6\},\{2,8\},\{2,4,8\}\} .
$$

It is nondegenerate in general and positive definite for $\mathbb{K}=\mathbb{R}$.

Remark. Since locally finite root systems have no nonzero imaginary roots, we need no longer distinguish between invariant and strictly invariant forms, as remarked in $\$ 2.7$.

Proof. Suppose (i) holds. Then it is of course well known that $\left|\left\langle R, R^{\vee}\right\rangle\right| \leq 4$. The existence of a unique normalized invariant form with the stated properties follows from [26, 4.2, 4.6, 4.14].

Conversely, suppose that (ii) holds with respect to the nondegenerate strictly invariant form $b$. Since $b$ is nondegenerate, $R^{\mathrm{im}}=\{0\}$. Hence, by $\S 2.8$, it remains to show that $R$ is locally finite, i.e., $|R \cap Y|<\infty$ for every finite-dimensional subspace $Y$ of $X$.

Since $R$ spans $X$, every finite-dimensional subspace lies in a finite-dimensional subspace $\tilde{Y}$ which is tight, i.e., $\tilde{Y}=\operatorname{Span}(\tilde{Y} \cap R)$. It is therefore no harm to assume that $Y$ itself is tight. One can then embed $Y$ in a finite-dimensional tight subspace $Y^{\prime}$ for which $b \mid Y^{\prime} \times Y^{\prime}$ is nondegenerate. Thus after replacing $Y$ by $Y^{\prime}$, 
we may assume $b$ is nondegenerate on $Y$. Let $\left\{\alpha_{1}, \ldots, \alpha_{n}\right\} \subset R \cap Y$ be a basis of $Y$. We claim that the linear map $\Phi: Y \rightarrow \mathbb{K}^{n}: y \mapsto\left(\left\langle y, \alpha_{i}^{\vee}\right\rangle\right)_{1 \leq i \leq n}$ is injective. Indeed, if $\Phi(y)=0$ we have $b\left(y, \alpha_{i}\right)=0$ by (2.16) and hence $y \in$ $Y \cap Y^{\perp}=\{0\}$. By assumption there exists $M \in \mathbb{N}$ such that $\left|\left\langle\beta, \alpha_{i}^{\vee}\right\rangle\right| \leq M$ for all $\beta \in R \cap Y$ and $1 \leq i \leq n$. Hence $\Phi(R \cap Y)$ is contained in the finite set $\left\{\left(m_{1}, \ldots, m_{n}\right) \in \mathbb{Z}^{n}:\left|m_{i}\right| \leq M\right\}$.

Remark. We note that an integral reflection system with a nondegenerate invariant or even strictly invariant form is in general not a locally finite root system. For example, consider $X=\mathbb{Q}^{4}$ with the hyperbolic quadratic form $q(x)=x_{1} x_{2}+$ $x_{3} x_{4}$ and let $b(x, y)=x_{1} y_{2}+x_{2} y_{1}+x_{3} y_{4}+x_{4} y_{3}$ be the associated bilinear form. Let $(R, X, s)$ be the integral reflection system of $\S 2.9$ with $R=\operatorname{Re}(R)$ and $R^{\text {re }}=\left\{\alpha \in \mathbb{Z}^{4}: q(\alpha)=1\right\}$. The form $b$ is strictly invariant and nondegenerate but $R$ is infinite, since the vectors $(1, n+1,-1, n)$ belong to $R$ for all $n \in \mathbb{Z}$.

\subsection{Reflection systems associated to bilinear forms}

Let $(R, X) \in \mathbf{S V}_{\mathbb{K}}$, and let ( | ) be a symmetric bilinear form on $X$. For $\alpha \in X$ we denote the linear form $x \mapsto(\alpha \mid x)$ by $\alpha^{b}$. Let $\Phi \subset\{\alpha \in R:(\alpha \mid \alpha) \neq 0\}$ be a subset, define ${ }^{\vee}: R \rightarrow X^{*}$ by

$$
\alpha^{\vee}:=\left\{\begin{array}{cl}
\frac{2 \alpha^{b}}{(\alpha \mid \alpha)} & \text { if } \alpha \in \Phi, \\
0 & \text { otherwise }
\end{array}\right.
$$

and define $s_{\alpha}$ by (2.7). Thus $s_{\alpha}$ is the orthogonal reflection in the hyperplane $\alpha^{\perp}$ if $\alpha \in \Phi$, and the identity otherwise. If $s_{\alpha}(R) \subset R$ and $s_{\alpha}(\Phi) \subset \Phi$ for all $\alpha \in R$, then $(R, X, s)$ is a reflection system with the given subset $\Phi$ as set of reflective roots. Indeed, the axioms $(\operatorname{ReS} 1)^{\vee},(\operatorname{ReS} 2)^{\vee},(\operatorname{ReS} 3)^{\vee}$ and $(\operatorname{ReS} 4)^{\vee}$ of $\S 2.3$ are easily verified. The bilinear form ( $\mid$ ) is invariant, and it is strictly invariant if and only if $R^{\mathrm{im}}=R \cap \operatorname{Rad}(\mid)$, i.e., ( $\mid$ ) is affine in the sense of $\S 5.1$.

Besides the locally finite root systems, many reflection systems are of this type. We mention as examples the root system associated to the geometric representation of a Coxeter group (\$2.10), the root system associated to root data (\$3.1.c) and the affine reflection systems (Lemma 5.3, Proposition 5.4). Other examples are the not necessarily crystallographic root systems, as for example defined in [20, 1.2], or the set $R=\Phi \cup\{0\}$ where $\Phi \subset \mathbb{R}^{2}$ is the set of vectors which enter into Tits' description of the Ree groups of type ${ }^{2} F_{4}$ [42, Figure 1 on p. 547]. We note that this $R$ is not reduced and that $W(R)$ is isomorphic to the dihedral group of order 16 . 


\subsection{Reflection systems à la Hée [18]}

Let $X$ be a $\mathbb{K}$-vector space with a basis $\left(\alpha_{i}\right)_{i \in I}$, and suppose $\left(s_{i}\right)_{i \in I}$ is a family of reflections with $s_{i}\left(\alpha_{i}\right)=-\alpha_{i}$ for all $i \in I$. Let $W$ be the subgroup of $\operatorname{GL}(X)$ generated by all $s_{i}, i \in I$, and put $R=\{0\} \cup W \cdot\left\{\alpha_{i}: i \in I\right\}$. It is straightforward to check that there exists a reflection system $(R, X, s)$ such that $s_{\alpha_{i}}=s_{i}$ for all $i \in I$ if and only if for all $w \in W, i, j \in I$ and $c \in \mathbb{K}^{\times}$the following condition is fulfilled:

$$
w\left(\alpha_{i}\right)=c \alpha_{j} \quad \Longrightarrow \quad w s_{i}=s_{j} w .
$$

In this case, the reflection $s_{\alpha}$ for $\alpha \in R^{\times}$is given by

$$
s_{\alpha}=w s_{i} w^{-1} \quad \text { for } \alpha=w\left(\alpha_{i}\right),
$$

so $R^{\text {re }}=R^{\times}$and $W=W(R)$.

Let now $\mathbb{K}$ be an ordered field, and suppose that $R=R_{+} \cup\left(-R_{+}\right)$where $R_{+}$ denotes the set of all $\alpha \in R$ which can be written in the form $\alpha=\sum_{i} c_{i} \alpha_{i}$ with all $c_{i} \geq 0$. Thus, $\left(I, X,\left(\alpha_{i}\right),\left(s_{i}\right)\right)$ is a "root basis" in the terminology of [18]. By $[18,2.13(\mathrm{~d})]$, the condition (2.23) is fulfilled and hence (2.24) defines a reflection system on $R$. We will establish (2.23) in another situation in Proposition 2.5.

By $[18,2.13]$ the Weyl group of $R$ is isomorphic to the Coxeter group $W$, as defined in $[18,1.3$ (c)]. In particular, by $[18,2.12$ (b)] every Coxeter system $(W, S)$ gives rise to a "root basis" in the sense of [18] over $\mathbb{K}=\mathbb{R}$, and hence to a real reflection system $R(W, S)$ whose Weyl group is isomorphic to $W$. In this setting, the reflection system $R(W, S)$ is also described in [14, V, §4] and [16]. By [16, Proposition 3.1], $R(W, S)$ is of the type considered in $\$ 2.9$ above.

\subsection{Lucas polynomials}

We will study the reflection system generated by two reflective roots in more detail and to this end first recall some facts about Lucas polynomials, see [27, Chapter 18].

Let $\mathbf{p}, \mathbf{q}$ be indeterminates. The Lucas polynomials $U_{n} \in \mathbb{Z}[\mathbf{p}, \mathbf{q}]$ are defined inductively for all $n \in \mathbb{N}$ by

$$
U_{0}=0, \quad U_{1}=1, \quad U_{n+1}=\mathbf{p} U_{n}-\mathbf{q} U_{n-1} \quad(n \geq 1) .
$$

Note that $U_{n}$ is homogeneous of degree $n-1$ provided $\mathbf{p}$ is given the degree 1 and $\mathbf{q}$ the degree 2. For negative indices, we define Lucas polynomials $U_{-n} \in \mathbb{Z}\left[\mathbf{p}, \mathbf{q}^{-1}\right]$ by

$$
U_{-n}(\mathbf{p}, \mathbf{q}):=-\mathbf{q}^{-n} U_{n}(\mathbf{p}, \mathbf{q})=-\mathbf{q}^{-1} U_{n}(\mathbf{p} / \mathbf{q}, 1 / \mathbf{q}),
$$

where the second formula follows from the homogeneity property of the $U_{n}$. It is easily seen that the recursion relations (2.25) then hold for all $n \in \mathbb{Z}$. 
The significance of the Lucas polynomials for the powers of a $2 \times 2$-matrix $A$ with coefficients in an arbitrary commutative ring $k$ is shown by the following formulas. Let us put

$$
p:=\operatorname{tr}(A), \quad q:=\operatorname{det}(A), \quad u_{n}:=U_{n}(p, q)
$$

for short and denote by $A^{\sharp}=\operatorname{tr}(A) I-A$ the adjoint matrix of $A$. Then for all $n \in \mathbb{N}$,

$$
\begin{aligned}
A^{n} & =u_{n} A-q u_{n-1} I \\
& =u_{n+1} I-u_{n} A^{\sharp} .
\end{aligned}
$$

For completeness, we indicate the proof. Clearly, (2.28) holds for $n=1$. If it holds for $n$, then multiplying by $A$ we obtain $A^{n+1}=u_{n} A^{2}-q u_{n-1} A=u_{n}(p A-$ $q I)-q u_{n-1} A$ (by Cayley-Hamilton) $=\left(p u_{n}-q u_{n-1}\right) A-q u_{n} I=u_{n+1} A-$ $q u_{n} I$ (by (2.25)). Now (2.29) follows from (2.28) because $A^{n}=u_{n}\left(p I-A^{\sharp}\right)-$ $q u_{n-1} I=\left(p u_{n}-q u_{n-1}\right) I-u_{n} A^{\#}=u_{n+1} I-u_{n} A^{\#}$.

If $A$ is invertible then $q=\operatorname{det}(A) \in k^{\times}$, and induction shows that (2.28) and (2.29) hold for all $n \in \mathbb{Z}$, where the $u_{n}$ are defined as before, but now for all $n \in \mathbb{Z}$.

Now specialize to the case where $k$ is a totally ordered commutative ring, e.g., $k=\mathbb{R}$, and $A \in \mathrm{SL}_{2}(k)$ and hence $q=\operatorname{det}(A)=1$. We identify $\mathbb{Z}$ with $\mathbb{Z} \cdot 1_{k} \subset k[13, \S 2.1$, Proposition 1]. Then (2.26) shows that

$$
u_{-n}=-u_{n} .
$$

We assume next that $|p|=|\operatorname{tr}(A)| \geq 2$, and put $\varepsilon=\operatorname{sgn}(p)$. Then the following estimate holds:

$$
\varepsilon^{n+1} u_{n} \geq \max \left(n, \varepsilon^{n} u_{n-1}+1\right) \quad \text { for all } n \in \mathbb{N} .
$$

We remark that these formulas are a compact version of $[18,(1.13)]$.

Proof. This is clear for $n=0$ because $u_{0}=0$ and $u_{-1}=-u_{1}=-1$. For the induction step, use (2.25) with $q=1$ :

$$
\varepsilon^{n+2} u_{n+1}=\varepsilon^{n+2}\left(p u_{n}-u_{n-1}\right)=|p| \varepsilon^{n+1} u_{n}-\varepsilon^{n} u_{n-1} .
$$

Since $|p| \geq 2$ and $\varepsilon^{n+1} u_{n} \geq n$ is in particular positive by induction, we have

$$
\begin{aligned}
\varepsilon^{n+2} u_{n+1} & \geq 2 \varepsilon^{n+1} u_{n}-\varepsilon^{n} u_{n-1}=\varepsilon^{n+1} u_{n}+\left(\varepsilon^{n+1} u_{n}-\varepsilon^{n} u_{n-1}\right) \\
& \geq \varepsilon^{n+1} u_{n}+1,
\end{aligned}
$$

from which the assertion follows. 
The lemma below is a simplification (in our situation) of [18, 1.21], see also [28, Proposition 5.1.11].

Lemma 2.3. Let $K$ be a field of characteristic $\neq 2$, let $A \in \mathrm{SL}_{2}(K)$ and $v \in K^{2}$ (column vector), and put $\tilde{A}:=\left(\begin{array}{ll}A & v \\ 0 & 1\end{array}\right) \in \mathrm{SL}_{3}(K)$. If $A \neq I$ (the $2 \times 2$ unit matrix), then $A$ and $\tilde{A}$ have the same order.

Proof. Let $B_{n}:=I+A+\cdots+A^{n-1}$. Induction shows that $\tilde{A}^{n}=\left(\begin{array}{cc}A^{n} & B_{n} v \\ 0 & 1\end{array}\right)$. If $A$ has 1 as an eigenvalue then $\operatorname{det} A=1$ implies that both eigenvalues of $A$ are 1 , and since $A \neq I$, it must be of the form $A=I+N$ where $N \neq 0=N^{2}$. Hence $A^{n}=I+n N$ and $B_{n}=n I+\left(\begin{array}{c}n \\ 2\end{array}\right) N=n\left(I+\frac{n-1}{2} N\right)$ for all $n \in \mathbb{N}$. Hence $A$ has infinite order if $K$ has characteristic zero, and order $p$ if $K$ has characteristic $p>2$, and so does $\tilde{A}$. If 1 is not an eigenvalue of $A$ then $A-I$ is invertible, and hence $B_{n}=(I-A)^{-1} \cdot\left(I-A^{n}\right)$, showing again that $A$ and $\tilde{A}$ have the same order.

The lemma fails for $A=\left(\begin{array}{ll}1 & 1 \\ 0 & 1\end{array}\right), v=\left(\begin{array}{l}0 \\ 1\end{array}\right)$ and $K$ of characteristic 2.

Lemma 2.4. Let $Y$ be a $\mathbb{K}$-vector space with basis $\{\alpha, \beta\}$ and let $s_{\alpha}, s_{\beta}$ be reflections with $s_{\alpha}(\alpha)=-\alpha$ and $s_{\beta}(\beta)=-\beta$. We denote by $D$ the subgroup of $\operatorname{GL}(Y)$ generated by $s_{\alpha}$ and $s_{\beta}$, and put $S=\{0\} \cup D \cdot\{\alpha, \beta\}$, cf. $\$ 2.10$.

(a) We identify $Y$ with $\mathbb{K}^{2}$ by means of the ordered basis $\alpha, \beta$ and let $A=s_{\alpha} s_{\beta}$. Define $a:=-\left\langle\beta, \alpha^{\vee}\right\rangle$ and $b:=-\left\langle\alpha, \beta^{\vee}\right\rangle$ where $\alpha^{\vee}, \beta^{\vee}$ are given by (2.7). Then

$$
A=\left(\begin{array}{cc}
a b-1 & -a \\
b & -1
\end{array}\right) \in \mathrm{SL}_{2}(\mathbb{K}) .
$$

Also, $D=N \rtimes\left\{\mathrm{Id}, s_{\alpha}\right\}=N \rtimes\left\{\mathrm{Id}, s_{\beta}\right\}$ (semidirect product) where $N$ denotes the cyclic group generated by $s_{\alpha} s_{\beta}$, so $D$ is a dihedral group.

(b) Put $u_{n}=U_{n}(\operatorname{tr}(A), \operatorname{det}(A))=U_{n}(a b-2,1)$ as in (2.27). Then

$$
S=\{0\} \cup\left\{ \pm A^{n} \alpha, \pm A^{n} \beta: n \in \mathbb{Z}\right\},
$$

where, for all $n \in \mathbb{Z}$,

$$
\begin{aligned}
A^{n} \alpha & =\left(u_{n+1}+u_{n}\right) \alpha+b u_{n} \beta, \\
-A^{n} \beta & =a u_{n} \alpha+\left(u_{n}+u_{n-1}\right) \beta .
\end{aligned}
$$

Proof. (a) is easily verified and well known, cf. [14, IV, §1.2]. 
(b) We have $\operatorname{tr}(A)=a b-2, \operatorname{det}(A)=1$ and hence $u_{n+1}=(a b-2) u_{n}-u_{n-1}$ by (2.25). Thus (2.29) yields

$$
\begin{aligned}
A^{n} & =\left(\begin{array}{cc}
u_{n+1} & 0 \\
0 & u_{n+1}
\end{array}\right)-u_{n}\left(\begin{array}{cc}
-1 & a \\
-b & a b-1
\end{array}\right) \\
& =\left(\begin{array}{cc}
u_{n+1}+u_{n} & -a u_{n} \\
b u_{n} & u_{n+1}-(a b-1) u_{n}
\end{array}\right) \\
& =\left(\begin{array}{cc}
u_{n+1}+u_{n} & -a u_{n} \\
b u_{n} & -u_{n}-u_{n-1}
\end{array}\right) .
\end{aligned}
$$

Now (2.36) and (2.37) follow immediately, and (2.35) is clear from the fact that $D=N \dot{U} N s_{\alpha}=N \dot{U} N s_{\beta}$.

Remark. We will later (in Lemma 3.6) apply this lemma and the following proposition to the situation where $(R, X, s)$ is a reflection system and $\alpha, \beta \in R^{\text {re }}$ are linearly independent reflective roots. Since $s_{\alpha}$ and $s_{\beta}$ leave $Y:=\mathbb{K} \alpha \oplus \mathbb{K} \beta$ invariant, the lemma is indeed applicable.

Let $H$ be the subgroup of $W(R) \subset \mathrm{GL}(X)$ generated by $s_{\alpha}$ and $s_{\beta}$. With $D$ defined as above, the restriction map $H \rightarrow D(\subset \mathrm{GL}(Y))$ is an isomorphism. Indeed, since $A \neq I$ the injectivity of the restriction map is an immediate consequence of Lemma 2.3. Moreover, in this case the set $S$ is the subsystem of $R$ generated by $\{\alpha, \beta\}$, cf. (2.14).

Proposition 2.5. With the assumptions and notations of Lemma 2.4, suppose furthermore that $a, b \in \mathbb{Z}$ and that $a b=0$ implies $a=0=b$. We also assume $a \geq|b| \geq 0$, which can always be achieved by switching $\alpha$ and $\beta$ and replacing $\beta$ by $-\beta$ if necessary. Then the condition (2.23) is fulfilled and hence $S$ is a reflection system. Moreover:

(a) If $\operatorname{tr}(A) \geq 2$, i.e., $a b \geq 4$, then $(\alpha, \beta) \cap S$ is infinite while $(\alpha,-\beta) \cap S=\emptyset$.

(b) If $\operatorname{tr}(A)<-2$, i.e., $a b<0$, both $(\alpha, \beta) \cap S$ and $(\alpha,-\beta) \cap S$ are infinite.

(c) In the remaining cases, i.e., $(a, b)=(0,0),(1,1),(2,1)$ or $(3,1), S$ is a finite reduced root system of rank 2 of type $\mathrm{A}_{1} \times \mathrm{A}_{1}, \mathrm{~A}_{2}, \mathrm{~B}_{2}$ and $\mathrm{G}_{2}$, respectively, with root basis $\alpha, \beta$. In particular, $(\alpha, \beta) \cap S$ is finite and $(\alpha,-\beta) \cap S=\emptyset$.

Proof. Note first that $a, b \in \mathbb{Z}$ implies all $u_{n} \in \mathbb{Z}$ and $S \subset \mathbb{Z} \alpha \oplus \mathbb{Z} \beta$. In (a) and (b) we will prove that, for all $n \in \mathbb{Z}$,

$$
u_{n+1}+u_{n} \neq 0, \quad \text { and } \quad u_{n}=0 \Longleftrightarrow n=0 .
$$


Assuming (2.38), we show that Condition (2.23) is fulfilled, and hence $S$ is a reflection system. Indeed, let $w \in D$ and consider $w \alpha$. By Lemma 2.4, we have $w=A^{n} s_{\alpha}^{i}$ for some $n \in \mathbb{Z}$ and $i \in\{0,1\}$, so $w \alpha=(-1)^{i}\left(\left(u_{n+1}+u_{n}\right) \alpha+b u_{n} \beta\right)$ by (2.36). Because of (2.38), $w \alpha \in \mathbb{K}^{\times} \beta$ is impossible, and if $w \alpha \in \mathbb{K}^{\times} \alpha$ then $b u_{n}=0$ forces $n=0$ by (2.38). Hence $w=s_{\alpha}^{i}$ and then obviously $w s_{\alpha}=s_{\alpha} w$ holds. The cases $w \beta \in \mathbb{K}^{\times} \alpha$ and $w \beta \in \mathbb{K}^{\times} \beta$ can be done similarly. Thus, for the proof of (a) and (b) it remains to establish the claims about the root intervals as well as (2.38). Because of (2.30) it is sufficient to show (2.38) for all $n \in \mathbb{N}$.

(a) We use (2.31) with $\varepsilon=1$. Thus $u_{n} \geq n$ for all $n \geq 0$ follows, which obviously implies (2.38) for $n \in \mathbb{N}$. Since $b>0$, (2.36) shows that the set $\left\{A^{n} \alpha: n \in \mathbb{N}_{+}\right\}$is infinite and contained in $(\alpha, \beta) \cap S$. Similarly, (2.37) shows that $-A^{n} \beta \in(\alpha, \beta) \cap S$ for all $n \geq 1$. For negative exponents, $A^{-n} \alpha=\left(u_{-n+1}+\right.$ $\left.u_{-n}\right) \alpha+b u_{-n} \beta=-\left(u_{n-1}+u_{n}\right) \alpha-b u_{n} \beta$ (by (2.30)) $\in-(\alpha, \beta) \cap S$, and similarly, $-A^{-n} \beta \in-(\alpha, \beta) \cap S$. Now (2.35) shows that $(\alpha,-\beta) \cap S=\emptyset$.

(b) Here we use (2.31) with $\varepsilon=-1$. This yields $-u_{2 n} \geq 2 n$ and $u_{2 n+1} \geq$ $2 n+1$ as well as $u_{2 n+1}+u_{2 n} \geq 1$ and $-\left(u_{2 n}+u_{2 n-1}\right) \geq 1$, for all $n \geq 1$. Again (2.38) follows. Also, (2.37) shows $A^{2 n} \beta=-a u_{2 n} \alpha-\left(u_{2 n}+u_{2 n-1}\right) \beta \in$ $(\alpha, \beta) \cap S$, for all $n \geq 1$. Similarly, since $u_{-n}=-u_{n}$ by $(2.30),-A^{-2 n} \beta=$ $-a u_{2 n} \alpha-\left(u_{2 n+1}+u_{2 n}\right) \beta \in(\alpha,-\beta) \cap S$, for all $n \geq 1$. Since $-a u_{2 n} \geq 2 n a$, there are infinitely many roots of these types.

(c) In the cases listed, one shows easily that $A$ has order 2, 3, 4, 6 and that $S$ is $\mathrm{A}_{1} \times \mathrm{A}_{1}, \mathrm{~A}_{2}, \mathrm{~B}_{2}, \mathrm{G}_{2}$, respectively, cf. [14]. In particular, $S$ is a reflection system. The details are left to the reader.

Remarks. (i) That, in case (a), $S$ is a reflection system, follows also from [18, 2.11 (ii), 2.13 (d)].

(ii) Because of (2.12), our assumption that $a b=0$ implies $a=0=b$ is necessary for $S$ to be a reflection system.

(iii) We summarize our results in the following table, where $a, b \in \mathbb{Z}$ satisfy the assumptions of the proposition:

\begin{tabular}{|c|c|c|c|c|c|c|c|c|}
\hline$a$ & $>0$ & 0 & 1 & 2 & 3 & 4 & 2 & $>0$ \\
\hline$b$ & $<0$ & 0 & 1 & 1 & 1 & 1 & 2 & $>0$ \\
\hline $\operatorname{tr}(A)$ & $<-2$ & -2 & -1 & 0 & 1 & 2 & 2 & $>2$ \\
\hline$S$ & $?$ & $\mathrm{~A}_{1} \times \mathrm{A}_{1}$ & $\mathrm{~A}_{2}$ & $\mathrm{~B}_{2}$ & $\mathrm{G}_{2}$ & $\mathrm{BC}_{1}^{(2)}$ & $\mathrm{A}_{1}^{(1)}$ & $\mathrm{hKM}$ \\
\hline$|(\alpha, \beta) \cap S|$ & $\aleph_{0}$ & 0 & 1 & 2 & 4 & $\boldsymbol{\aleph}_{0}$ & $\boldsymbol{\aleph}_{0}$ & $\aleph_{0}$ \\
\hline$|(\alpha,-\beta) \cap S|$ & $\aleph_{0}$ & 0 & 0 & 0 & 0 & 0 & 0 & 0 \\
\hline
\end{tabular}


Here $\mathrm{BC}_{1}^{(2)}$ and $\mathrm{A}_{1}^{(1)}$ denote the sets of real roots of the corresponding affine Kac-Moody algebras [28, p. 452], and hKM indicates that $S$ is the set of real roots of a hyperbolic Kac-Moody algebra, cf. [28, p. 454]. The case $a>0>b$ does not seem to have appeared "naturally" in Lie algebras.

\subsection{More examples}

(a) Restriction along subsystems. Let $U \subset R$ be a subsystem of a pre-reflection system $R$. Then it follows easily that $R$ together with the modified definition

$$
\tilde{s}_{\alpha}:= \begin{cases}s_{\alpha} & \text { for } \alpha \in U, \\ \text { Id } & \text { for } \alpha \in R \backslash U,\end{cases}
$$

defines a new pre-reflection system $(R, X, \tilde{s})$ which we denote by $R^{U}$ or simply by $\tilde{R}$ and call the restriction of $R$ along $U$. Clearly, the reflective roots of $\tilde{R}$ are those of $U$, i.e., $\operatorname{Re}\left(R^{U}\right)=\operatorname{Re}(U)$. If $R$ is a reflection system, then so is $R^{U}$.

(b) Quotients of classical root systems. Let $I$ be a set and $X_{I}=\bigoplus_{i \in I} \mathbb{K} \varepsilon_{i}$ the free $\mathbb{K}$-vector space on the set $I$. We will use the following description of the classical root systems, cf. [26, 8.1]:

$$
\begin{aligned}
& \dot{\mathrm{A}}_{I}=\left\{\varepsilon_{i}-\varepsilon_{j}: i, j \in I\right\}, \quad \mathrm{D}_{I}=\dot{\mathrm{A}}_{I} \cup\left\{ \pm \varepsilon_{i} \pm \varepsilon_{j}: i \neq j\right\}, \\
& \mathrm{B}_{I}=\mathrm{D}_{I} \cup\left\{ \pm \varepsilon_{i}: i \in I\right\}, \quad \mathrm{C}_{I}=\mathrm{D}_{I} \cup\left\{ \pm 2 \varepsilon_{i}: i \in I\right\}, \\
& \mathrm{BC}_{I}=\mathrm{B}_{I} \cup \mathrm{C}_{I} .
\end{aligned}
$$

The notation $\dot{\mathrm{A}}_{I}$ (instead of $\mathrm{A}_{I}$ ) serves to indicate the fact that the span of $\dot{\mathrm{A}}_{I}$ has codimension 1 in $X_{I}$. In all other cases, except $\mathrm{D}_{I}$ for $|I|=1$, the span of $R$ is $X_{I}$. For a subset $J$ of $I$ we put $K=I \backslash J$ and define

$$
\begin{aligned}
& \mathrm{BC}_{I}(J)=\mathrm{B}_{I} \cup\left\{ \pm 2 \varepsilon_{j}: j \in J\right\}=\mathrm{BC}_{I} \backslash\left\{ \pm 2 \varepsilon_{k}: k \in K\right\}, \\
& \mathrm{DC}_{I}(J)=\mathrm{D}_{I} \cup\left\{ \pm 2 \varepsilon_{j}: j \in J\right\}=\mathrm{C}_{I} \backslash\left\{ \pm 2 \varepsilon_{k}: k \in K\right\},
\end{aligned}
$$

cf. [26, 12.18]. Our notation is motivated by the fact that $\mathrm{BC}_{I}(J)$ interpolates between the root systems $\mathrm{B}_{I}=\mathrm{BC}_{I}(\emptyset)$ and $\mathrm{BC}_{I}=\mathrm{BC}_{I}(I)$, while $\mathrm{DC}_{I}(J)$ does the same for $\mathrm{D}_{I}=\mathrm{DC}_{I}(\emptyset)$ and $\mathrm{C}_{I}=\mathrm{DC}_{I}(I)$. By [26, 12.15], a quotient of a classical root system by a full subsystem is either again a classical root system or isomorphic to $\mathrm{BC}_{I}(J)$ or $\mathrm{DC}_{I}(J)$ for suitable $I, J$.

Let $R=\mathrm{BC}_{I}(J)$ or $\mathrm{DC}_{I}(J)$. For $0 \neq \alpha \in R \cap\left(X_{J} \cup X_{K}\right)$ let $s_{\alpha}$ be the usual reflection with respect to $\alpha$ in the root system $\mathrm{BC}_{I}$. Then $s_{\alpha}$ leaves $R$ as well as $R \cap\left(X_{J} \cup X_{K}\right)$ invariant. Since (ReS3) and (ReS4) hold in $\mathrm{BC}_{I}$, it is immediate 
that $R$ becomes a reflection system for which

$$
\begin{aligned}
\operatorname{Re}(R) & =R \cap\left(X_{J} \cup X_{K}\right)= \begin{cases}\mathrm{BC}_{J} \cup \mathrm{B}_{K} & \text { if } R=\mathrm{BC}_{I}(J), \\
\mathrm{C}_{J} \cup \mathrm{D}_{K} & \text { if } R=\mathrm{DC}_{I}(J),\end{cases} \\
R^{\mathrm{im}} & =\{0\} \cup\left\{ \pm \varepsilon_{j} \pm \varepsilon_{k}: j \in J, k \in K\right\} .
\end{aligned}
$$

It is symmetric, integral and saturated, but $R=\mathrm{BC}_{I}(J)$ is not reduced. It is immediately seen that $\mathrm{DC}_{I}(J)$ is a subsystem of $\left.\operatorname{BC}_{I}(J), s\right)$.

There exists a second structure of a reflection system on $\mathrm{BC}_{I}(J)$ as follows. The subset $U:=\mathrm{C}_{J} \cup \mathrm{B}_{K}$ is a subsystem of $R=\mathrm{BC}_{I}(J)$ and hence restriction along $U$ gives rise to the new reflection system

$$
\tilde{R}=\mathrm{BC}_{I}(J)^{U}
$$

whose reflective and imaginary roots are

$$
\operatorname{Re}(\tilde{R})=\mathrm{C}_{J} \cup \mathrm{B}_{K}, \quad \tilde{R}^{\mathrm{im}}=R^{\mathrm{im}} \cup\left\{ \pm \varepsilon_{j}: j \in J\right\},
$$

with $R^{\mathrm{im}}$ as in (2.43). The reflection system $\tilde{R}$ is integral and reduced but not saturated. Note that $\operatorname{DC}_{I}(J)$ is a subsystem of both $R$ and $\tilde{R}$.

(c) Root data in the sense of Moody-Pianzola. Let $\mathscr{D}=\left(A, \Pi, \Pi^{\vee}, V, V^{\vee},\langle\rangle,\right)$ be a set of root data over $\mathbb{K}$ in the sense of [28, 5.1], let $W$ be its Weyl group, and let $\Sigma=W \cdot \Pi$ be the set of real roots of $\mathscr{D}$. Also, let $R$ be the root string closure of $\Sigma$ [28, 5.8]. For $\alpha \in \Sigma$, let $s_{\alpha}=r_{\alpha}$ be the reflection defined by $\alpha$ as in [28, 5.2], while for $\phi \in R \backslash \Sigma$ put $s_{\phi}=$ Id. Then $R$ is a symmetric, reduced, saturated and integral reflection system in the vector space $X \subset V$ spanned by $\Pi$, with $\Sigma$ as its set of reflective roots. This follows easily from [28, Chapter 5]. Let us point out that in particular the roots of a Kac-Moody algebra are examples of root data and hence of reflection systems.

(d) Weight systems. Let $\mathfrak{g}$ be a Kac-Moody algebra over $\mathbb{K}[28,4.1]$ with root system $\Delta=\Delta^{\text {re }} \cup \Delta^{\mathrm{im}} \subset \mathfrak{h}^{*}$ in the usual notation. We do not assume that $\mathfrak{h}$ is minimally realized in the sense of $[28,4.2]$ and hence allow for example that $\mathrm{g}$ is a finite-dimensional reductive Lie algebra with a split semisimple part. Let $(M, \pi)$ be an integrable $g$-module $[28,6.1]$ with weight system $P(M)$. We have $(R, X) \in \mathbf{S V}$ for

$$
R:=\{0\} \cup \Delta \cup P(M)
$$

and $X=\operatorname{span}_{\mathbb{K}}(R)$. For $\alpha \in \Delta^{\text {re }}$ let $s_{\alpha} \in \mathfrak{h}^{*}$ be the usual reflection. It then follows from [28, Proposition 4.1.5, 4.1.7 and 6.1.10] that $s_{\alpha}(R)=R$ and $s_{\alpha}\left(\Delta^{\mathrm{re}}\right)=$ $\Delta^{\text {re }}$. Also, by [28, Proposition 4.1.2], (ReS4) ${ }^{\vee}$ is satisfied. Hence $R$ is a reflection system with $R^{\text {re }}=\Delta^{\text {re }}$. It is also integral: For $\alpha \in \Delta^{\text {re }}$ we have $\left\langle\Delta, \alpha^{\vee}\right\rangle \subset \mathbb{Z}$ by 
[28, Proposition 4.1.7 and Proposition 4.1.9] and $\left\langle P(M), \alpha^{\vee}\right\rangle \subset \mathbb{Z}$ by [28, Proposition 6.1.4]. We note that $R$ is symmetric if and only if

$$
-P(M) \subset R \text {. }
$$

For example, (2.46) is fulfilled if $M$ is isomorphic to its dual module. We will discuss this further in $\S 3.1(\mathrm{~d}, \mathrm{e})$.

(e) Integrable roots in Lie algebras with split toral subalgebras. Let $L$ be a Lie algebra over $\mathbb{K}$ with a split toral subalgebra $T$. This means that $T$ is a subalgebra of $L$ with the following property: $L=\bigoplus_{\alpha \in R} L_{\alpha}(T)$ where, for any $\phi \in T^{*}$, we put $L_{\phi}(T)=\{x \in L:[t, x]=\phi(t) x$ for all $t \in T\}$ and where $R=\left\{\phi \in T^{*}\right.$ : $\left.L_{\phi}(T) \neq 0\right\}$. We denote by $R^{\text {int }}$ the subset of integrable roots, i.e., those $\alpha \in R$ for which there exists an $\mathfrak{s} \Upsilon_{2}$-triple $\left(e_{\alpha}, h_{\alpha}, f_{\alpha}\right) \in L_{\alpha} \times T \times L_{-\alpha}$ such that the adjoint maps ad $e_{\alpha}$ and ad $f_{\alpha}$ are locally nilpotent.

Let $X=\operatorname{Span}_{\mathbb{K}}(R) \subset T^{*}$, and for $\alpha \in R^{\text {int }}$ define $\alpha^{\vee} \in X^{*}$ by $\alpha^{\vee}(\phi)=$ $\phi\left(h_{\alpha}\right)$. It is then a straightforward exercise in $\mathfrak{s l}_{2}$-representation theory to verify that $(R, X)$ is an integral pre-reflection system with $R^{\text {re }}=R^{\text {int }}$. We note that many of the examples above arise in this way, e.g., locally finite root systems [33], Kac-Moody root systems [23, 28] or the examples in (d).

\section{Partial root systems}

Lemma 3.1. Let $R$ be a pre-reflection system, let $\alpha \in R^{\mathrm{re}}, \beta \in R$, and assume that $a:=-\left\langle\beta, \alpha^{\vee}\right\rangle \in \mathbb{Z}$. Consider the $\alpha$-string through $\beta$, defined by

$$
\mathbb{S}(\beta, \alpha):=(\beta+\mathbb{Z} \alpha) \cap R,
$$

and let $\mathbb{Z}(\beta, \alpha):=\{i \in \mathbb{Z}: \beta+i \alpha \in R\}$.

(a) $s_{\alpha}$ leaves $\mathbb{S}(\beta, \alpha)$ invariant and corresponds to the reflection $i \mapsto a-i$ of $\mathbb{Z}(\beta, \alpha)$ about the point $a / 2$.

(b) $\mathbb{Z}(\beta, \alpha)$ is bounded if and only if it is bounded on one side. In this case, we put $-q=\min \mathbb{Z}(\beta, \alpha)$ and $p=\max \mathbb{Z}(\beta, \alpha)$ and then have $p, q \in \mathbb{N}$ and $p-q=a=-\left\langle\beta, \alpha^{\vee}\right\rangle$.

(c) The following conditions are equivalent:

(i) $\mathbb{S}(\beta, \alpha)$ contains no gaps, i.e., $\mathbb{Z}(\beta, \alpha)$ is a finite interval in $\mathbb{Z}$ or equals $\mathbb{Z}$,

(ii) for all $\gamma \in \mathbb{S}(\beta, \alpha)$ and all integers $i$ between 0 and $\left\langle\gamma, \alpha^{\vee}\right\rangle$ we have $\gamma-i \alpha \in \mathbb{S}(\beta, \alpha)$

(iii) for all $\gamma \in \mathbb{S}(\beta, \alpha),\left\langle\gamma, \alpha^{\vee}\right\rangle>0$ implies $\gamma-\alpha \in R$ and $\left\langle\gamma, \alpha^{\vee}\right\rangle<0$ implies $\gamma+\alpha \in R$. 
Proof. (a) follows immediately from $s_{\alpha}(\beta+i \alpha)=\beta+(a-i) \alpha$.

(b) The first statement is clear from (a) and the symmetry of $\mathbb{Z}(\beta, \alpha)$ about the point $a / 2$. We have $p, q \in \mathbb{N}$ because $0 \in \mathbb{Z}(\beta, \alpha)$. Now $-q$ and $p$ are exchanged by the map $i \mapsto a-i$, whence $-q=a-p$.

(c) The implication (i) $\Longrightarrow$ (ii) follows from the fact that $\mathbb{S}(\beta, \alpha)$ is stable under $s_{\alpha}$ and $s_{\alpha}(\gamma)=\gamma-\left\langle\gamma, \alpha^{\vee}\right\rangle \alpha$, and (ii) $\Longrightarrow$ (iii) is trivial. Assume that (iii) holds but that $\mathbb{S}(\beta, \alpha)$ has a gap, say with left endpoint $\gamma=\beta+l \alpha$ and right endpoint $\delta=\beta+r \alpha$, so that $l+1<r$. Since $\gamma+\alpha$ and $\delta-\alpha$ do not belong to $R$, we have

$$
\begin{aligned}
& 0 \leq\left\langle\gamma, \alpha^{\vee}\right\rangle=\left\langle\beta+l \alpha, \alpha^{\vee}\right\rangle=\left\langle\beta, \alpha^{\vee}\right\rangle+2 l, \\
& 0 \geq\left\langle\delta, \alpha^{\vee}\right\rangle=\left\langle\beta+r \alpha, \alpha^{\vee}\right\rangle=\left\langle\beta, \alpha^{\vee}\right\rangle+2 r .
\end{aligned}
$$

Subtracting these inequalities yields the contradiction $l \geq r$.

Lemma 3.2. Let $(R, X)$ be an integral pre-reflection system. Then there exists a unique integral reflection system $(\tilde{R}, X)$ such that

(i) $\tilde{R}$ contains $R$ as a subsystem, and $\tilde{R}^{\mathrm{re}}=R^{\mathrm{re}}$;

(ii) for every $\alpha \in R^{\mathrm{re}}$ and $\tilde{\beta} \in \tilde{R}$ the root string $(\tilde{\beta}+\mathbb{Z} \alpha) \cap \tilde{R}$ is without gaps;

(iii) $\tilde{R}$ is the smallest with respect to inclusion among all reflection systems satisfying (i) and (ii).

If $R$ is symmetric or a reflection system, then so is $\tilde{R}$.

Proof. For $R$ the set of real roots associated to a set of root data, see $\$ 2.12$ (d), this is proven in $[28, \S 5.8]$ where $\tilde{R}$ is called the "root string closure" of $R$. The construction given there also works in our more general setting.

Definition 3.3. A partial root system is an integral reflection system $(R, X, s)$ with the following additional properties:

(PRS1) (Signs) For all $\alpha, \beta \in R^{\text {re }}, \operatorname{sgn}\left\langle\alpha, \beta^{\vee}\right\rangle=\operatorname{sgn}\left\langle\beta, \alpha^{\vee}\right\rangle$, where $\operatorname{sgn}(n)$ is defined as zero, 1 , or -1 according to whether $n$ is zero, positive, or negative.

(PRS2) (Root string property) For all $\alpha \in R^{\mathrm{re}}, \beta \in R$, the root string $\mathbb{S}(\beta, \alpha)$ is finite and without gaps.

(PRS3) (Partial closure) Whenever $\alpha, \beta \in R^{\mathrm{re}},\left\langle\beta, \alpha^{\vee}\right\rangle \geq 0$ and $\alpha+\beta \in R$ then $\alpha+\beta \in R^{\text {re }}$.

By a morphism between partial root systems, we mean a morphism of the underlying reflection systems. Thus the partial root systems form a full subcategory, denoted PRS, of the category ReS of reflection systems. 
Let us point out that we do not assume that $R$ be reduced as defined in $\S 2.4$. We note that (PRS1) is equivalent to:

(PRS1) $^{\prime}$ For all $\alpha, \beta \in R^{\mathrm{re}},\left\langle\alpha, \beta^{\vee}\right\rangle>0 \Longleftrightarrow\left\langle\beta, \alpha^{\vee}\right\rangle>0$.

Indeed, (PRS1) $)^{\prime}$ implies $\left\langle\alpha, \beta^{\vee}\right\rangle<0 \Longleftrightarrow\left\langle\beta, \alpha^{\vee}\right\rangle<0$ since $(-\beta)^{\vee}=-\beta^{\vee}$ by $(\operatorname{ReS} 3)^{\vee}$, while $\left\langle\alpha, \beta^{\vee}\right\rangle=0 \Longleftrightarrow\left\langle\beta, \alpha^{\vee}\right\rangle=0$ holds for all reflection systems by (2.12).

We also note that for $\alpha, \beta$ as in (PRS3), we have $\alpha+\beta \in R^{\times}$, else $\left\langle\beta, \alpha^{\vee}\right\rangle=$ $-\left\langle\alpha, \alpha^{\vee}\right\rangle=-2$. In particular, (PRS3) always holds if $R^{\text {re }}=R^{\times}$. However, in general $\operatorname{Re}(R)=R^{\mathrm{re}} \cup\{0\}$ is not a closed subset of $R$.

The following fact will be useful:

$$
\alpha \in R^{\mathrm{re}} \text { and } n \alpha \in R \text { for } n \geq 2 \quad \Longrightarrow \quad n \alpha \in R^{\mathrm{re}}, n=2 \text { and } s_{\alpha}=s_{2 \alpha} .
$$

Indeed, by (2.10) it suffices to show that $n \alpha \in R^{\text {re }}$. The $\alpha$-string through $\alpha$ contains no gaps by (PRS2), hence $i \alpha \in R$ for $1 \leq i \leq n-1$. As $\left\langle i \alpha, \alpha^{\vee}\right\rangle=2 i>0$, induction and (PRS3) shows $n \alpha \in R^{\text {re }}$.

\subsection{Examples}

Examples of partial root systems are the so-called EARS or, more generally, some of the affine reflection systems, see Corollary 4.7 (b). Other examples are some of the reflection systems of $\S 2$, discussed below.

(a) Locally finite root systems. By well-known facts $[14,26]$, the (locally) finite root systems in the usual sense are precisely those (locally) finite partial root systems for which $\operatorname{Re}(R)=R$.

(b) Quotients of classical root systems. We consider the three types of integral reflection systems defined in $\$ 2.12$ (b). We use the notation introduced there. Since the reflections are always the ones induced from $\mathrm{BC}_{I}$, it suffices to indicate the reflective and imaginary roots:

$$
\begin{aligned}
& R=\mathrm{BC}_{I}(J), \operatorname{Re}(R)=\mathrm{BC}_{J} \cup \mathrm{B}_{K}, \\
& \tilde{R}=R^{\mathrm{im}}=\{0\} \cup\left\{ \pm \varepsilon_{j} \pm \varepsilon_{k}: j \in J, k \in K\right\}, \\
& S=\mathrm{BC}_{I}(J), \operatorname{Re}(\tilde{R})=\mathrm{C}_{J} \cup \mathrm{B}_{K}, \quad \tilde{R}^{\mathrm{im}}=R^{\mathrm{im}} \cup\left\{ \pm \varepsilon_{j}: j \in J\right\}, \operatorname{Re}(S)=\mathrm{C}_{J} \cup \mathrm{D}_{K}, \quad S^{\mathrm{im}}=R^{\mathrm{im}} .
\end{aligned}
$$

Then $R$ is not a partial root system (unless $J$ or $K$ is empty), because for $j \in J$ and $k \in K$ we have $\varepsilon_{j}, \varepsilon_{k} \in R^{\text {re }}$ and $\left\langle\varepsilon_{j}, \varepsilon_{k}^{\vee}\right\rangle=0$ but $\varepsilon_{j}+\varepsilon_{k} \in R^{\mathrm{im}}$. On the other hand, $\tilde{R}$ and $S$ are symmetric partial root systems. Indeed, (PRS1) holds since it does so in $\mathrm{BC}_{I}$. To show (PRS2) for $\tilde{R}$, let $\alpha \in \tilde{R}^{\text {re }}, \beta \in \tilde{R}$ and denote by $\mathbb{S}(\beta, \alpha)$ 
and $\mathbb{S}^{*}(\beta, \alpha)$ the root strings in $\tilde{R}$ and $\mathrm{BC}_{I}$, respectively. Then $\mathbb{S}(\beta, \alpha)$ is finite since it is contained in $\mathbb{S}^{*}(\beta, \alpha)$. Because

$$
\mathrm{BC}_{I} \backslash \tilde{R}=\left\{ \pm 2 \varepsilon_{k}: k \in K\right\},
$$

the structure of root strings in root systems shows that the only possibility for $\mathbb{S}(\beta, \alpha)$ to have a gap is a situation where $\beta+(i \pm 1) \alpha \in \tilde{R}$ and $\beta+i \alpha= \pm 2 \varepsilon_{k}$ for some $k \in K$. But by [14, VI, $\S 1.3$, Remarque], the longer roots are at the end of root strings in $\mathrm{BC}_{I}$, contradiction. The proof for $S$ is analogous. Finally, it is easily seen that (PRS3) holds by inspecting all possibilities for two reflective roots $\alpha, \beta$ with $\left\langle\beta, \alpha^{\vee}\right\rangle \geq 0$.

(c) Root data. The reflection system associated to a set of root data $\mathscr{D}$ in $\$ 2.12$ (c) is a symmetric partial root system. Indeed, we have already seen that $R$ is symmetric and integral. Property (PRS1) ${ }^{\prime}$ is proven in [28, Proposition 5.2.8 (ii)]. By construction, $R$ is the root string closure of $\Sigma$, so the root string through $\beta$ in direction of a real root $\alpha \in \Sigma$ is without gaps, by Lemma 3.1 (c). It is also finite: Since $\Sigma=W \cdot \Pi$, we may assume that $\alpha=\alpha_{i} \in \Pi$. If, say, $\beta \in R_{+}$then $\beta+n \alpha_{i} \in R$ is only possible for $n \geq 0$, by the "Wonderful Union Property". Thus root strings are bounded on one side, and hence bounded, by Lemma 3.1 (b). It remains to verify (PRS3), so let $\alpha, \beta \in R^{\text {re }}=\Sigma$, let $\left\langle\beta, \alpha^{\vee}\right\rangle \geq 0$, and assume that $\gamma:=\alpha+\beta \in R^{\text {im }}$. For an imaginary root of a Kac-Moody algebra, it is known [28, Corollary (i) of Proposition 5.8.9] that every integer multiple is again an imaginary root. Using the covering map of [28, Proposition 5.1.1], it follows that the same is true in $R$; in particular, $2 \gamma \in R$. Now consider the $\alpha$-string $2 \gamma-q \alpha, \ldots, 2 \gamma, \ldots, 2 \gamma+p \alpha$ through $2 \gamma$. We have $q-p=\left\langle 2 \gamma, \alpha^{\vee}\right\rangle \geq 4$, in particular, $2 \gamma-2 \alpha \in R$. But $2 \gamma-2 \alpha=2 \beta \notin R$ by [28, Proposition 5.1.6], contradiction.

(d) Weight systems. Let $R=\{0\} \cup \Delta \cup P(M)$ be the integral reflection system of $\$ 2.12$ (d) associated to a Kac-Moody algebra $g$ and an integral $g$-module $M$ with weight system $P(M)$. We claim that $R$ is a partial root system with $R^{\text {re }}=\Delta^{\text {re }}$ if and only if

$$
\begin{aligned}
& \mathbb{S}(\lambda, \alpha) \text { is finite for } \lambda \in P(M) \text { and } \alpha \in \Delta^{\mathrm{re}}, \text { and } \\
& \alpha, \beta \in \Delta^{\mathrm{re}},\left\langle\beta, \alpha^{\vee}\right\rangle \geq 0 \text { and } \alpha+\beta \in P(M) \text { implies } \alpha+\beta \in R^{\mathrm{re}} .
\end{aligned}
$$

Indeed, from (c) we know that (PRS1) and (PRS2) hold for $\alpha, \beta \in \Delta^{\text {re }}$. By [28, Proposition 6.2.1] we also know that $\mathbb{S}(\lambda, \alpha)$ for $\lambda \in P(M)$ does not contain gaps, and hence (PRS2) holds in general if and only if (3.2) is fulfilled. Finally, for (PRS3) assume $\alpha, \beta \in R^{\mathrm{re}}=\Delta^{\mathrm{re}},\left\langle\beta, \alpha^{\vee}\right\rangle \geq 0$ and $\alpha+\beta \in R$. If $\alpha+\beta \in \Delta$ then $\alpha+\beta \in \Delta^{\mathrm{re}}$, as we have seen in (c). The remaining case, i.e., $\alpha+\beta \in P(M)$, is condition (3.3). 
(e) Root systems of classical Lie superalgebras. Recall that a simple finitedimensional Lie superalgebra $L$ over an algebraically closed field $\mathbb{K}$ of characteristic 0 is called classical if the representation of the even part $L_{\overline{0}}$ on the odd part $L_{\overline{1}}$ is completely reducible, and is called basic classical if it is classical and has an invariant nondegenerate even symmetric bilinear form. In the following we assume $L_{\overline{1}} \neq 0$. For a classical Lie superalgebra the even part $L_{\overline{0}}$ is necessarily reductive, hence a Kac-Moody algebra, say with root system $\Delta$. We are therefore in the setting of example (d) above. We claim that $R=\{0\} \cup \Delta \cup P\left(L_{\overline{1}}\right)$ is a partial root system with $R^{\text {re }}=\Delta$, which is symmetric if $L$ is not of type $\mathbf{P}(n)$, in particular if $L$ is classical. To see this, it suffices by finite dimensionality of $L$ to verify the condition (3.3). To do so, we will use the description of the root systems in classical Lie algebras given in [22, Proposition 2.1.2 and 2.5.4]. This will also show symmetry of $R$ (of course, for classical Lie superalgebras this also follows from the existence of a nondegenerate invariant bilinear form).

1. $\mathbf{A}(m, n), m, n \in \mathbb{N}, m \neq n$ or $m=n \geq 1$ : Let $S$ be the 3-graded root system $\mathrm{A}_{m+n+1}^{m+1}$ in the notation of $[26,17.8]$, thus $S=\mathrm{A}_{m+n+1}$ and $S_{0}=\mathrm{A}_{m} \times \mathrm{A}_{n}$. Then $R$ is the restriction of $S$ along the full subsystem $S_{0}$, cf. $\$ 2.12$ (a), from which (3.3) easily follows.

2. $\mathbf{B}(m, n), m \in \mathbb{N}, n \in \mathbb{N}_{+}: R$ is isomorphic (as reflection system and hence also as partial root system) to the partial root system $\tilde{R}=\mathrm{BC}_{I}(J)$ of (b) above, with $|I|=m+n$ and $|J|=n$.

3. $\mathbf{C}(n), n \in \mathbb{N}, n \geq 2$ : Here $R$ is isomorphic to the partial root system $S=$ $\mathrm{DC}_{I}(J)$ of (b) above, with $|I|=n$ and $|J|=n-1$.

4. $\mathbf{D}(m, n), m, n \in \mathbb{N}_{+}, m \geq 2: \quad R$ is isomorphic to the partial root system $S=\mathrm{DC}_{I}(J)$ of (b) above, with $|I|=m+n$ and $|J|=n$.

5. $\mathbf{D}(2,1 ; \alpha)$ : Here $R \subset \mathbb{K} \varepsilon_{1} \oplus \mathbb{K} \varepsilon_{2} \oplus \mathbb{K} \varepsilon_{3}$ with $R^{\text {re }}=\left\{ \pm 2 \varepsilon_{i}: 1 \leq i \leq 3\right\}$ and $R^{\mathrm{im}}=\{0\} \cup\left\{ \pm \varepsilon_{1} \pm \varepsilon_{2} \pm \varepsilon_{3}\right\}$. Obviously (3.3) holds by inspection.

6. $\mathbf{F}(4)$ : We have $R \subset \mathbb{K} \varepsilon_{1} \oplus \mathbb{K} \varepsilon_{2} \oplus \mathbb{K} \varepsilon_{3} \oplus \mathbb{K} \delta$ with $R^{\text {re }}=\left\{ \pm \varepsilon_{i}, \pm \varepsilon_{i} \pm \varepsilon_{j}\right.$ : $i \neq j\} \cup\{ \pm \delta\}=\mathrm{B}_{3}^{\times} \cup \mathrm{A}_{1}^{\times}$and $R^{\mathrm{im}}=\left\{\frac{1}{2}\left( \pm \varepsilon_{1} \pm \varepsilon_{2} \pm \varepsilon_{3} \pm \delta\right)\right\} \cup\{0\}$. Again, (3.3) follows by inspection.

7. G(3): It is straightforward to check (3.3). The details are left to the reader.

8. $\mathbf{P}(n): R=\mathrm{C}_{n+1} \backslash\left\{-2 \varepsilon_{i}: 1 \leq i \leq n+1\right\}$ with $R=\left\{\varepsilon_{i}-\varepsilon_{j}: i \neq j\right\}=\mathrm{A}_{n}^{\times}$. Condition (3.3) holds since $\operatorname{Re}(R)$ is closed in $R$ (it consists of the trace-0elements).

9. $\mathbf{Q}(n): R=\mathrm{A}_{n}=\operatorname{Re}(R)=R^{\mathrm{im}}$, so $R$ is a root system and hence in particular a partial root system. 
(f) Let $\Delta$ be a root system in the sense of [12, p. 110ff.] which is furthermore integral, i.e., for all $\alpha \in \Delta$ with $(\alpha \mid \alpha)>0$ and all $\beta \in \Delta$, we have $2(\beta \mid \alpha) /(\alpha \mid \alpha) \in \mathbb{Z}$. Let $X$ be the span of $\Delta$ (in the vector space in which $\Delta$ lives), and put $R:=\Delta \cup\{0\}$ and $R^{\text {re }}=\{\alpha \in R:(\alpha \mid \alpha)>0\}$. Define $\alpha^{\vee}$ by (2.22) and note that $s_{\alpha}(R)=R$ holds by axiom (S4) (c) of [12]. Since $s_{\alpha} R^{\text {re }}=R^{\text {re }}$ is obvious, we are in the setting of $\$ 2.9$ and see that $R$ is an integral reflection system. We claim that $(R, X, s)$ is a symmetric partial root system. Indeed, axiom (PRS1) is clear from (2.22), and (PRS3) follows from the fact that $(\alpha+\beta \mid \alpha+\beta)=(\alpha \mid \alpha)+\left\langle\beta, \alpha^{\vee}\right\rangle(\alpha \mid \alpha)+(\beta \mid \beta)>0$ whenever $\alpha, \beta \in R^{\text {re }}$ and $\left\langle\beta, \alpha^{\vee}\right\rangle \geq 0$. Finally, (PRS2) follows easily from (S3), (S4) (d) and Lemma 3.1 (c), and symmetry of $R$ is axiom (S2). Note that the axioms (S1) and (S5)-(S7) of [12] are not required, and axiom (S3) is only needed in case one of the roots $\alpha, \beta$ is real. As a consequence, the root systems of symmetrizable Kac-Moody-Borcherds algebras, which satisfy Bardy's axioms except possibly (S1) and are integral by [12, Proposition 1.1, Remarque], are in particular examples of partial root systems.

\subsection{T-nilpotence}

The concepts which we introduce now generalize a definition due to Tits in the setting of real roots of Kac-Moody algebras ([43, 3.2], see also [36, 6.2.4]). They were called pre-nilpotence respectively nilpotence by Tits and will be called $\mathrm{T}$-(pre)nilpotence here, in order to distinguish them from the notions of nilpotence introduced in $\S 1$.

Let $R$ be a reflection system with Weyl group $W(R)$, and let $P \subset R$ be a positive system in the sense of $[26,10.10 .5]$, i.e., a positive subset with $P \cup(-P)=R$. A subset $A \subset R^{\text {re }}$ is called T-prenilpotent with respect to $P$ if

(i) $A$ is finite and prenilpotent,

(ii) there exist $w, w^{\prime} \in W(R)$ such that $w(A) \subset P$ and $w^{\prime}(A) \subset-P$.

Recall from Proposition 1.6 that condition (i) is equivalent to

(i) ${ }^{\prime} A^{c}$ is finite and does not contain 0 ,

where $A^{c}$ denotes the closure of $A$, cf. (1.1).

A subset $A \subset R^{\text {re }}$ is T-nilpotent with respect to $P$ if it is T-prenilpotent and, moreover, satisfies

(iii) $A$ is closed with respect to sums of two roots in $R^{\text {re }}$, i.e., if $\alpha, \beta \in A$ and $\alpha+\beta \in R^{\mathrm{re}}$ then $\alpha+\beta \in A$. 
We now relate T-nilpotence to nilpotence in locally finite root systems and also show that for the root systems of Kac-Moody algebras, the above definition of $T$ prenilpotence is consistent with the definition of prenilpotence given by Tits [43].

Lemma 3.4. (a) Let $R$ be a locally finite root system and $A$ a finite subset of $R$. Then the notions of (pre)nilpotence and of $T$-(pre)nilpotence (with respect to any positive system) for A coincide.

(b) Let $R$ be the reflection system determined by a set of root data as in $\$ 2.12$ (c), let $P=R_{+}$be the positive system associated with $\Pi$, and let $A \subset \Sigma=R^{\mathrm{re}}$ be a subset satisfying (ii) of \$3.2. Then condition (i) holds as well, and $A^{c} \subset \Sigma$.

Proof. (a) First suppose $A$ is (pre)nilpotent. Since $A$ is finite and $R$ is locally finite, there exists a finite subsystem $S$ of $R$ containing $A$, and $P \cap S$ is a positive system in $S$. Now $A^{c}$ is strictly positive by (1.31). Hence, there exists some positive system $P^{\prime}$ of $S$ containing $A^{c}$ [26, Proposition 10.13(a)]. In finite root systems, positive systems are conjugate under the Weyl group. Hence there exist $w, w^{\prime} \in W(S)$ with $w\left(P^{\prime}\right) \subset P \cap S$ and $w^{\prime}\left(P^{\prime}\right) \subset(-P) \cap S$, and since $w, w^{\prime}$ are induced from elements of $W(R)$, we see that condition (ii) of $\$ 3.2$ holds. If $A$ is nilpotent, then it is in particular closed, so condition (iii) of $\$ 3.2$ holds. Thus, any (pre)nilpotent subset is T-(pre)nilpotent. For the converse, it suffices to show that a set $A$ satisfying the weaker closure condition (iii) is actually closed. This is a consequence of the fact that $R^{\text {re }}=R^{\times}$and that locally finite root systems have the partial sum property, see $[26,10.2,10.3]$.

(b) It suffices to prove condition (i) $)^{\prime}$ of $\$ 3.2$. After replacing $A$ by $w(A)$ and $w^{\prime}$ by $w$ we may assume $A \subset \Sigma_{+}$and $w(A) \subset \Sigma_{-}=-\Sigma_{+}$. Let $\alpha_{1}, \ldots, \alpha_{n} \in A$ and $\beta=\alpha_{1}+\cdots+\alpha_{n} \in R$. Since $A$ is a subset of the strictly positive set $R_{+}^{\times}$, we have $0 \neq \beta \in R_{+}^{\times}$. Moreover, $w\left(\alpha_{i}\right) \in R_{-}^{\times}$and hence also $w(\beta)$ belongs to $R_{-}^{\times}$. Since the Weyl group stabilizes $R_{+}^{\mathrm{im}}, \beta$ cannot be imaginary. This proves $A^{c} \subset \Sigma=R^{\mathrm{re}}$; in particular, $0 \notin A^{c}$. Finally, $S_{w}:=\left\{\alpha \in \Sigma_{+}: w(\alpha) \in \Sigma_{-}\right\}$ is finite (of cardinality equal to the length of $w$ ) by [28, Proposition 5.2.3], so $A^{c} \subset S_{w}$ is finite as well.

Obviously, if $A$ is T-prenilpotent (with respect to some $P$ ) then it is prenilpotent. Our aim for the rest of this section is to show a converse of this, namely: For a partial root system $R$ and $A=\{\alpha, \beta\} \subset R^{\text {re }}$ of cardinality at most 2, prenilpotence of $A$ (in the sense of 1.5) implies T-prenilpotence with respect to any positive system $P$ of scalar type (Theorem 3.7). We begin with two lemmas.

Lemma 3.5. Let $R$ be a partial root system, let $\alpha, \beta \in R^{\mathrm{re}}$ and let $\left\langle\beta, \alpha^{\vee}\right\rangle \geq 0$. Then the closed root interval $[\alpha, \beta]=R \cap \mathbb{N}_{+}[\{\alpha, \beta\}]$ is contained in $R^{\mathrm{re}}$ and is 
one of the following:

$$
\{\alpha, \beta\}, \quad\{\alpha, 2 \alpha, \beta\}, \quad\{\alpha, \beta, 2 \beta\}, \quad\{\alpha, \alpha+\beta, \beta\}, \quad\{\alpha, 2 \alpha, \alpha+\beta, \beta, 2 \beta\} .
$$

In particular, $\{\alpha, \beta\}$ is prenilpotent and $(\alpha, \beta) \subset\{\alpha+\beta\}$. If $(\alpha, \beta) \neq \emptyset$, i.e., $\alpha+\beta \in R^{\mathrm{re}}$ by (PRS3), then $\alpha$ and $\beta$ are indivisible in $R^{\mathrm{re}}$.

Proof. (a) As a first step, we show: If $\xi$ belongs to the open root interval $(\alpha, \beta)$ and thus $\xi=m \alpha+n \beta$ where $m, n \in \mathbb{N}_{+}$then

$$
k \alpha+l \beta \in R \quad \text { for all } k, l \in \mathbb{Z} \text { with }|k| \leq m \text { and }|l| \leq n \text {, and }
$$

$m, n \in\{1,2\}$.

Indeed, $\left\langle\xi, \beta^{\vee}\right\rangle=m\left\langle\alpha, \beta^{\vee}\right\rangle+n\left\langle\beta, \beta^{\vee}\right\rangle=m\left\langle\alpha, \beta^{\vee}\right\rangle+2 n \geq 2 n$. Now consider the $\beta$-string through $\xi$ :

$$
\xi-q \beta, \ldots, \xi-\beta, \xi, \xi+\beta, \ldots, \xi+p \beta .
$$

Since $q \geq q-p=\left\langle\xi, \beta^{\vee}\right\rangle \geq 2 n$, we have $\xi-i \beta \in R$ for $i=0, \ldots, 2 n$, equivalently,

$$
m \alpha+j \beta \in R \quad \text { for }|j| \leq n .
$$

Interchanging the roles of $\alpha$ and $\beta$, it follows in the same way that

$$
i \alpha+n \beta \in R \quad \text { for }|i| \leq m .
$$

By applying (3.6) repeatedly to roots of the form (3.7) where $1 \leq i \leq m$, and vice versa, we see that (3.4) holds. This together with (3.1) implies (3.5).

(b) We now show that

$$
2 \alpha+2 \beta \notin R .
$$

Assume to the contrary that $\delta:=2 \alpha+2 \beta \in R$. By (3.4), we then have also $2 \alpha+\beta$ and $\alpha+2 \beta$ in $R$, as well as $\gamma:=\alpha+\beta \in R$. Moreover, $\gamma \in R^{\text {re }}$ by (PRS3), and therefore also $\delta=2 \gamma \in R^{\text {re }}$, by (3.1). Now consider the $\delta$ string through $\alpha+2 \beta$. Then $(\alpha+2 \beta)+\delta=3 \alpha+4 \beta \notin R$ by (3.5), while $(\alpha+2 \beta)-\delta=-\alpha \in R$ but $(\alpha+2 \beta)-2 \delta=-(3 \alpha+2 \beta) \notin R$ by (3.5). This shows $p=0$ and $q=1$ where $p, q$ are as in Lemma 3.1 (b) for $\mathbb{S}(\alpha+2 \beta, \delta)$. It follows that $\left\langle\alpha+2 \beta, \delta^{\vee}\right\rangle=q-p=1$. Now $2 \delta^{\vee}=\gamma^{\vee}$ by $(\operatorname{ReS} 3)^{\vee}$ so we obtain

$$
2=2\left\langle\alpha+2 \beta, \delta^{\vee}\right\rangle=\left\langle\alpha+2 \beta, \gamma^{\vee}\right\rangle=\left\langle\gamma, \gamma^{\vee}\right\rangle+\left\langle\beta, \gamma^{\vee}\right\rangle=2+\left\langle\beta, \gamma^{\vee}\right\rangle .
$$

This implies $\left\langle\beta, \gamma^{\vee}\right\rangle=0$ and therefore, by integrality, also $0=\left\langle\gamma, \beta^{\vee}\right\rangle=$ $\left\langle\alpha, \beta^{\vee}\right\rangle+2 \geq 2$, contradiction. 
(c) For the statement concerning $(\alpha, \beta)$, it remains in view of (3.5) and by symmetry to show that $2 \alpha+\beta \notin R$.

Assume to the contrary that $\varepsilon:=2 \alpha+\beta \in R$. By (3.4), we have $2 \alpha \in R$ hence $2 \alpha \in R^{\mathrm{re}}$ by (3.1), and therefore $\varepsilon \in R^{\mathrm{re}}$ by (PRS3). Furthermore,

$$
\left\langle\varepsilon,(2 \alpha)^{\vee}\right\rangle=\frac{1}{2}\left\langle\varepsilon, \alpha^{\vee}\right\rangle=\frac{1}{2}\left\langle 2 \alpha+\beta, \alpha^{\vee}\right\rangle=2+\frac{1}{2}\left\langle\beta, \alpha^{\vee}\right\rangle>0,
$$

whence also $\left\langle 2 \alpha, \varepsilon^{\vee}\right\rangle>0$, and even $\left\langle 2 \alpha, \varepsilon^{\vee}\right\rangle \geq 2$, by integrality. Therefore, the $\varepsilon$ string through $2 \alpha$ contains the root $2 \alpha-2 \varepsilon=-(2 \alpha+2 \beta) \in R$ which contradicts what we proved in (b). This establishes $(\alpha, \beta) \subset\{\alpha+\beta\}$.

(d) Now let $\alpha+\beta \in R$, and assume that $\alpha$ is not indivisible in $R^{\text {re }}$, say, $\alpha=2 \alpha^{\prime}$ where $\alpha^{\prime}$ is in $R^{\text {re }}$. Then $\left\langle\alpha^{\prime}, \beta^{\vee}\right\rangle \geq 0$, and $\alpha+\beta=2 \alpha^{\prime}+\beta \in\left(\alpha^{\prime}, \beta\right)$, contradicting what we already proved. Likewise, $\beta$ must be indivisible. Now assume $2 \alpha \in R$, put again $\gamma=\alpha+\beta$, and consider the $\gamma$-string through $2 \alpha$. By (3.4), we have $\alpha-\beta \in R$, and $2 \alpha+\gamma=3 \alpha+\beta \notin R$ by (3.5). Hence $p=0$ and $q \geq 1$ for this string. Since $q-p=q=\left\langle 2 \alpha, \gamma^{\vee}\right\rangle$ is even, it follows that $q \geq 2$, whence $2 \alpha-2 \gamma=-2 \beta \in R$. This completes the proof.

Lemma 3.6. Let $R$ be a partial root system and suppose $\alpha, \beta \in R^{\text {re }}$ satisfy $\left\langle\beta, \alpha^{\vee}\right\rangle$. $\left\langle\alpha, \beta^{\vee}\right\rangle \leq 3$. Then $T:=R \cap(\mathbb{Z} \alpha+\mathbb{Z} \beta)$ is a finite root system of rank 2 in the vector space $Y$ spanned by $\alpha$ and $\beta$ and $T^{\times} \subset R^{\mathrm{re}}$.

Proof. Since $(-\alpha)^{\vee}=-\alpha^{\vee}$ we may replace $\alpha$ by $-\alpha$ if necessary and thus assume that $\left\langle\beta, \alpha^{\vee}\right\rangle \leq 0$. Then also $\left\langle\alpha, \beta^{\vee}\right\rangle \leq 0$ by (PRS1). Lemma 3.5, applied to $\alpha$ and $-\beta$, shows that

$$
\left\langle\beta, \alpha^{\vee}\right\rangle \leq 0 \Longrightarrow(\alpha,-\beta) \subset\{\alpha-\beta\} \subset R^{\mathrm{re}} \text { and } 2 \alpha-\beta, \alpha-2 \beta \notin R .
$$

We also remark that $\alpha$ and $\beta$ are linearly independent. Indeed, assuming $s \alpha+t \beta=$ 0 for some $s, t \in \mathbb{K}$ implies $2 s+t\left\langle\beta, \alpha^{\vee}\right\rangle=0=s\left\langle\alpha, \beta^{\vee}\right\rangle+2 t$. The integral matrix $\left(\underset{\left\langle\alpha, \beta^{\vee}\right\rangle}{2}\left\langle\stackrel{\left\langle\beta, \alpha^{\vee}\right\rangle}{2}\right)\right.$ has determinant $\geq 1$, whence $s=t=0$.

(a) As a first step in the proof, we show that it is no restriction of generality to assume that $(\alpha,-\beta)=\emptyset$. Indeed, assume $(\alpha,-\beta) \neq \emptyset$, hence $\gamma:=\alpha-\beta \in R^{\text {re }}$ by (3.9), and put $a:=-\left\langle\beta, \alpha^{\vee}\right\rangle$ and $b:=-\left\langle\alpha, \beta^{\vee}\right\rangle$ for short. We claim that

$$
a b \in\{0,1\} .
$$

If this were not the case then $a b \in\{2,3\}$ because $0 \leq a b \leq 3$. Possibly after interchanging $\alpha$ and $\beta$ we may assume $b=1$ and $a \in\{2,3\}$. Then $s_{\beta}(\alpha)=$ $\alpha+\beta \in R^{\mathrm{re}}$, and $\left\langle(\alpha+\beta), \alpha^{\vee}\right\rangle=2-a \leq 0$. Hence by (3.9), applied to $\alpha$ and $\alpha+\beta$ instead of $\alpha$ and $\beta$, we have $2 \alpha-(\alpha+\beta)=\alpha-\beta \notin R$, contradiction. This proves (3.10), which is obviously equivalent to $a=b \in\{0,1\}$ because $a, b \in \mathbb{N}$ 
and $\operatorname{sgn}(a)=\operatorname{sgn}(b)$. Then $\left\langle\gamma, \beta^{\vee}\right\rangle=\left\langle\alpha, \beta^{\vee}\right\rangle-2=-b-2 \in\{-2,-3\}$, and hence also $\left\langle\beta, \gamma^{\vee}\right\rangle<0$, by (PRS1). Moreover, $(\gamma,-\beta)=\emptyset$, otherwise $\gamma-\beta=\alpha-2 \beta \in R$, contradicting (3.9).

We claim next that $\left\langle\beta, \gamma^{\vee}\right\rangle \cdot\left\langle\gamma, \beta^{\vee}\right\rangle \leq 3$, i.e., that $\left\langle\beta, \gamma^{\vee}\right\rangle=-1$. Consider the $\gamma$-string through $\beta$. We have $\beta-\gamma=2 \beta-\alpha \notin R$ by (3.9), $\beta+\gamma=\alpha \in R$, but $\beta+2 \gamma=2 \alpha-\beta \notin R$, again by (3.9). Thus $q=0$ and $p=1$ for this string, which implies $\left\langle\beta, \gamma^{\vee}\right\rangle=q-p=-1$, as desired.

Thus we see that the pair $(\gamma, \beta)$ satisfies the assumptions made on $(\alpha, \beta)$, and additionally has $\gamma-\beta \notin R$, i.e., $(\gamma,-\beta)=\emptyset$. Clearly, $\mathbb{Z} \gamma+\mathbb{Z} \beta=\mathbb{Z} \alpha+\mathbb{Z} \beta$ holds as well. We can therefore replace $\alpha$ by $\gamma$ and then have (a).

(b) Assume now that $\alpha$ has been replaced by $\gamma$ as above if necessary, and that therefore $(\alpha,-\beta)=\emptyset$. From example (b) of $\S 2.5$, it follows that $T$ is a subsystem of $R$ in the subspace $Y$.

As before, we may assume that $a:=-\left\langle\beta, \alpha^{\vee}\right\rangle \geq 0, b:=\left\langle\alpha, \beta^{\vee}\right\rangle \geq 0$, and $a \geq b$. Let $S$ be the subsystem generated by $\{\alpha, \beta\}$. We are therefore in the situation considered in Lemma 2.4. By Proposition 2.5 (c), $S$ is a finite reduced root system of rank 2 , and clearly $S \subset T$. Let

$$
T_{\text {ind }}^{\times}:=\left\{\xi \in T^{\times}: \xi / 2 \notin T^{\times}\right\}
$$

be the set of roots of $T^{\times}$which are indivisible in $T^{\times}$and note that $\xi / 2 \in R$ is possible for an element of $T_{\text {ind }}^{\times}$. We put $T_{\text {ind }}=\{0\} \cup T_{\text {ind }}^{\times}$. As $\alpha / 2 \notin \mathbb{Z} \alpha \oplus \mathbb{Z} \beta$, we have $\alpha \in T_{\text {ind }}^{\times}$and likewise $\beta \in T_{\text {ind }}^{\times}$. Let $H$ be the subgroup of $W(R)$ generated by $s_{\alpha}$ and $s_{\beta}$, cf. the remark in Lemma 2.4. Then $H$ leaves $\mathbb{Z} \alpha \oplus \mathbb{Z} \beta$ invariant, and therefore stabilizes $T$ and $T_{\text {ind }}$ and, of course, $S$. Now Formula (2.14) shows that $S \subset T_{\text {ind }}$. We claim that in fact

$$
S=T_{\text {ind }},
$$

i.e., that $E:=T_{\text {ind }} \backslash S$ is empty.

Indeed, $(\alpha,-\beta)=\emptyset$ implies $T=T_{+} \cup T_{-}$where $T_{+}=T \cap(\mathbb{N} \alpha+\mathbb{N} \beta)$ and $T_{-}=T \cap(-\mathbb{N} \alpha-\mathbb{N} \beta)$. Accordingly, $E=E_{+} \cup E_{-}$where $E_{ \pm}=E \cap T_{ \pm}$. Since $T_{\text {ind }}$ and $S$ are $H$-stable so is $E$. Let us show that in fact $E_{+}$is $H$-stable. It suffices to do this for the generators of $H$. Let $\xi=m \alpha+n \beta \in E_{+}$. Then necessarily $m>0$ and $n>0$; otherwise, say, $\xi=m \alpha$ where $m \in\{1,2\}$ by (2.10). But $\alpha \in S^{\times}$and $2 \alpha \notin T_{\text {ind }}$, so $\xi \notin E$. Now

$$
s_{\alpha}(\xi)=(a n-m) \alpha+n \beta, \quad s_{\beta}(\xi)=m \alpha+(b m-n) \beta
$$

implies that $s_{\alpha}(\xi)$ and $s_{\beta}(\xi)$ belong to $E_{+}$.

Assume $E_{+} \neq \varnothing$ and let $\xi=m \alpha+n \beta \in E_{+}$be a minimal element with respect to the lexicographic order $\geq$ given by the ordered basis $(\alpha, \beta)$ of the vector 
space $Y$. Such an element exists because $E_{+} \subset \mathbb{N} \alpha+\mathbb{N} \beta$. Then $s_{\alpha}(\xi) \geq \xi$, $s_{\beta}(\xi) \geq \xi$ and (3.12) imply $a n-m \geq m$ and $b m-n \geq n$, which yields ban $\geq$ $2 b m \geq 4 n$ and hence $a b \geq 4$, contradicting our assumption $a b \leq 3$. Thus $E_{+}=\emptyset$. One proves in the same way that $E_{-}=\emptyset$, whence (3.11) holds.

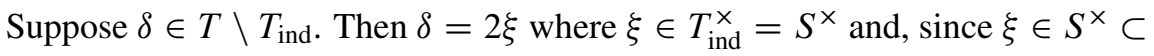
$R^{\mathrm{re}}$, we have $2 \xi \in R^{\mathrm{re}}$ by (3.1). This shows that $T$ is finite (with $\left|T^{\times}\right| \leq 2\left|S^{\times}\right|$), and that $T^{\times}=T^{\text {re }}$. Hence by $\S 2.8, T$ is a finite root system of rank 2 in $Y$.

Theorem 3.7. Let $R$ be a partial root system, and let $\alpha, \beta \in R^{\mathrm{re}}$. Moreover, let $P$ be a positive system of $R$ such that $P \cap R^{\mathrm{re}}$ is of scalar type in the sense that there exists a linear form $h$ on $X$ such that $h\left(R^{\mathrm{re}}\right)$ is contained in an ordered subfield of $\mathbb{K}$ and $h\left(P^{\mathrm{re}}\right)>0$. Then the following conditions are equivalent:

(i) $\{\alpha, \beta\}$ is T-prenilpotent with respect to $P$,

(ii) $\{\alpha, \beta\}$ is prenilpotent,

(iii) $(\alpha, \beta)$ is finite,

(iv) $\left\langle\beta, \alpha^{\vee}\right\rangle \geq 0$ or $\left\langle\beta, \alpha^{\vee}\right\rangle\left\langle\alpha, \beta^{\vee}\right\rangle \leq 3$.

If these conditions hold, then $[\alpha, \beta]=\{\alpha, \beta\}^{c}$, the closure of $\{\alpha, \beta\}$ in $R$, is finite of cardinality $\leq 6$, nilpotent of class $\leq 5$, and contained in $R^{\text {re }}$. Moreover, $(\alpha, \beta) \neq \varnothing$ if and only if $\alpha+\beta \in R$.

Proof. (i) $\Longrightarrow$ (ii) is evident from the definition in $\$ 3.2$.

(ii) $\Longrightarrow$ (iii): $[\alpha, \beta]$ is finite by Proposition 1.6, and hence so is $(\alpha, \beta) \subset[\alpha, \beta]$.

(iii) $\Longrightarrow$ (iv): Assume $-a:=\left\langle\beta, \alpha^{\vee}\right\rangle \leq 0$ hence also $-b:=\left\langle\alpha, \beta^{\vee}\right\rangle \leq 0$, but $a b \geq 4$. Then $(\alpha, \beta)$ is infinite by Proposition 2.5 (a).

(iv) $\Longrightarrow$ (i): There are two possibilities:

(a) $\left\langle\beta, \alpha^{\vee}\right\rangle \geq 0$. Prenilpotence of $[\alpha, \beta]$ was shown in Lemma 3.5. We have $R^{\text {re }}=P^{\text {re }} \dot{\cup}\left(-P^{\text {re }}\right)$. Hence, possibly after replacing both $\alpha$ and $\beta$ by their negatives, it suffices to consider the following cases:

Case 1. $\{\alpha, \beta\} \subset P^{\text {re }}$,

Case 2. $\{\alpha,-\beta\} \subset P^{\text {re }}$.

Let us first assume that $\left\langle\beta, \alpha^{\vee}\right\rangle=\left\langle\alpha, \beta^{\vee}\right\rangle=0$. Then $s_{\alpha}(\beta)=\beta$ and $s_{\beta}(\alpha)=\alpha$ while $s_{\alpha}(\alpha)=-\alpha$ and $s_{\beta}(\beta)=-\beta$. Hence in Case $1, w=\operatorname{Id}$ and $w^{\prime}=$ $s_{\alpha} s_{\beta}$ satisfies the requirements of $\S 3.2$ (ii), and in Case 2, we can put $w=s_{\beta}$ and $w^{\prime}=s_{\alpha}$.

Next, let $\left\langle\beta, \alpha^{\vee}\right\rangle>0$, and consider Case 1. Suppose to the contrary that $w\{\alpha, \beta\} \not \subset-P^{\text {re }}$ for all $w \in W(R)$. Then in particular $s_{\alpha}\{\alpha, \beta\}=\left\{-\alpha, s_{\alpha}(\beta)\right\} \not \subset$ 
$-P^{\text {re }}$ and $s_{\beta}\{\alpha, \beta\}=\left\{s_{\beta}(\alpha),-\beta\right\} \not \subset-P^{\text {re }}$, and therefore $s_{\alpha}(\beta) \in P^{\text {re }}$ and $s_{\beta}(\alpha) \in P^{\text {re }}$. By our assumption on $P^{\text {re }}$, this means

$$
h\left(s_{\alpha}(\beta)\right)=h(\beta)-\left\langle\beta, \alpha^{\vee}\right\rangle h(\alpha)>0,
$$

i.e., $h(\beta)>\left\langle\beta, \alpha^{\vee}\right\rangle h(\alpha)>0$, and likewise $h(\alpha)>\left\langle\alpha, \beta^{\vee}\right\rangle h(\beta)>0$. But then $h(\beta)>\left\langle\beta, \alpha^{\vee}\right\rangle\left\langle\alpha, \beta^{\vee}\right\rangle h(\beta)$ and therefore $1>\left\langle\beta, \alpha^{\vee}\right\rangle\left\langle\alpha, \beta^{\vee}\right\rangle>0$, contradicting integrality. This establishes Case 1 .

Now consider Case 2. Then $s_{\alpha}(\beta)=\beta+\left\langle\beta, \alpha^{\vee}\right\rangle(-\alpha) \in\left(-P^{\mathrm{re}}\right)$ since $\left\langle\beta, \alpha^{\vee}\right\rangle>0$, and similarly $s_{\beta}(\alpha)=\alpha+\left\langle\alpha, \beta^{\vee}\right\rangle(-\beta) \in P^{\text {re }}$. Hence $s_{\alpha}\{\alpha, \beta\} \subset$ $\left(-P^{\mathrm{re}}\right)$ while $s_{\beta}\{\alpha, \beta\} \subset P^{\mathrm{re}}$.

(b) $\left\langle\beta, \alpha^{\vee}\right\rangle\left\langle\alpha, \beta^{\vee}\right\rangle \leq 3$. Then $T=R \cap(\mathbb{Z} \alpha \oplus \mathbb{Z} \beta)$ is a finite root system of rank 2 by Lemma 3.6; in particular, $\alpha$ and $\beta$ are linearly independent. Also, $[\alpha, \beta]$ is closed and does not contain 0 , and is thus a positive subset of $T$, cf. (1.4). By [26, Proposition 10.13(a)], which also holds in our setting, it is contained in a positive system, say $T_{+}$, of $T$. Now $P \cap T$ is also a positive system of $T$. By conjugacy of positive systems in finite root systems, there exist $w, w^{\prime} \in W(T)$ with $w\left(T_{+}\right)=P \cap T$ and $w^{\prime}\left(T_{+}\right) \subset-(P \cap T)$. Since $w$ and $w^{\prime}$ are induced by elements of $W(R)$, the assertion follows. The remaining statements follow from Lemma 3.5 and Lemma 1.3.

Corollary 3.8. For the partial root systems determined by root data, in particular, for the root systems of Kac-Moody algebras, the notions of prenilpotent pair and T-prenilpotent pair of roots (relative to the standard positive system) coincide.

Proof. Let $R$ be the partial root system determined by a set of root data as in $\S 3.1$ (b). Then the standard positive system $P$ is of scalar type with respect to the usual height function $h$. Hence a prenilpotent pair $\{\alpha, \beta\}$ is T-prenilpotent with respect to $P$ by Theorem 3.7. The converse was shown in Lemma 3.4 (b).

Remark. It is an open problem to extend this result to sets of more than two roots.

\section{Extensions}

Lemma 4.1. Let $f:(R, X) \rightarrow(S, Y)$ be a morphism of pre-reflection systems and put $Z:=\operatorname{Ker}(f), R_{0}:=R \cap Z$ and $R_{1}:=\{0\} \cup(R \backslash Z)$. We denote by $W_{i}(R)$ the subgroups of $W(R)$ generated by $\left\{s_{\alpha}: \alpha \in R_{i}\right\}$.

(a) $R_{0}$ and $R_{1}$ are $W(R)$-stable subsystems of $R$ in the subspaces $X_{i}$ spanned by $R_{i}, i=0,1$.

(b) $W(R)$ stabilizes $Z$ and $W_{1}(R)$ fixes $Z$ pointwise. The restriction map $w \mapsto$ $w \mid X_{1}$ is an isomorphism $W_{1}(R) \stackrel{\cong}{\longrightarrow} W\left(R_{1}\right)$. 
(c) $\bar{R}:=f(R)$ is a subsystem of $S$ in the vector subspace $\bar{X}$ of $Y$ spanned by $f(R)$. There is a unique surjective homomorphism

$$
\phi=W(f): W(R) \rightarrow W(\bar{R})
$$

satisfying $\phi\left(s_{\alpha}\right)=\left(s_{f(\alpha)} \mid \bar{X}\right)$ for all $\alpha \in R$, and

$$
f \circ w=\phi(w) \circ f
$$

for all $w \in W(R)$.

(d) $\phi$ induces an exact sequence

$$
0 \longrightarrow V \stackrel{\text { inc }}{\longrightarrow} W_{1}(R) \stackrel{\phi_{1}}{\longrightarrow} W(\bar{R}) \longrightarrow 1
$$

where $\phi_{1}=\phi \mid W_{1}(R)$ and $V:=W_{1}(R) \cap \operatorname{Ker}(\phi)$ is isomorphic to a subgroup of the additive group of $\operatorname{Hom}(\bar{X}, Z)$.

(e) If $f(R)=S$, an invariant form $b_{S}$ on $Y$ lifts to an invariant form $b_{R}$ on $X$ defined by $b_{R}(x, y)=b_{S}(f(x), f(y))$, and if $b_{S}$ is strictly invariant, so is $b_{R}$.

(f) If $R$ is a reflection system, the subgroups $W_{i}(R)$ are normal and their product is $W(R)$.

Proof. (a) From (2.4) it is clear that $W(R)$ stabilizes $R_{1}, R_{0}$ and $Z$. This implies that $R_{1}$ and $R_{0}$ are subsystems of $R$

(b) If $\alpha \in R_{1}$ then (2.8) shows $\left\langle Z, \alpha^{\vee}\right\rangle=0$ and hence $s_{\alpha}$ is the identity on $Z$ by (2.7). From $R=R_{1} \cup R_{0}$ we have $X=X_{1}+X_{0}$ and clearly $X_{0} \subset Z$. Hence the restriction map $W_{1}(R) \rightarrow W\left(R_{1}\right)$, which is obviously surjective, is injective as well.

(c) It is immediate from (2.3) that $f(R)$ is a reflection subsystem of $S$. Since $W(R)$ stabilizes $Z$, every $w \in W(R)$ induces a unique transformation $\phi(w)$ of $\bar{X} \cong X / Z$ satisfying (4.1), and $\phi: W(R) \rightarrow \operatorname{GL}(\bar{X})$ is obviously a group homomorphism. From (2.4) we see that $\phi\left(s_{\alpha}\right)=s_{f(\alpha)} \mid \bar{X} \in W(\bar{R})$, whence $\phi(W(R))=W(\bar{R})$.

(d) From the definition of $W_{1}(R)$ it is clear that also $\phi: W_{1}(R) \rightarrow W(\bar{R})$ is surjective, so we have (4.2). An element $v \in V$ induces the identity both on $Z$ (by (b)) and on $\bar{X} \cong X / Z$. Hence, it has the form $v(x)=x+h_{v}(f(x))(x \in X)$, for a unique $h_{v} \in \operatorname{Hom}(\bar{X}, Z)$. It is easily checked that the map $v \mapsto h_{v}$ is an injective homomorphism of $V$ into the additive $\operatorname{group} \operatorname{Hom}(\bar{X}, Z)$. 
(e) For $w \in W(R)$ we have $b_{R}(w(x), w(y))=b_{S}(\phi(w) f(x), \phi(w) f(y))=$ $b_{R}(x, y)$ by (4.1), so $b_{R}$ is invariant. If $b_{S}$ is strictly invariant then $R^{\mathrm{im}} \subset \operatorname{Rad} b_{R}$ holds because of (2.5), so $b_{R}$ is also strictly invariant.

(f) (ReS4)' shows that $W_{i}(R)$ is normal in $W(R)$. Now $W(R)=W_{1}(R)$. $W_{0}(R)$ follows from $R=R_{1} \cup R_{0}$.

Definition 4.2. Let $f:(R, X) \rightarrow(S, Y)$ be a morphism of pre-reflection systems with $f(R)=S$, and let $S^{\prime} \subset S$ be a subsystem spanning $Y$. A partial section of $f$ over $S^{\prime}$ is a morphism $g:\left(S^{\prime}, Y\right) \rightarrow(R, X)$ of pre-reflection systems such that $f \circ g=\operatorname{Id}_{Y}$. Note that $X=\operatorname{Ker}(f) \oplus g(Y)$ because of $f \circ g=\operatorname{Id}_{Y}$. Naturally, a section of $f$ is a partial section of $f$ over all of $S$.

As we will see in Lemma 4.3, a partial section of $f$ leads to a partial section of the exact sequence (4.2) over $W\left(S^{\prime}\right)$. Moreover, while sections of $f$ need not exist, Proposition 4.4 shows that partial sections always exist in $\mathbf{R e S}$ and, under some additional assumptions, lead to a splitting of the exact sequence (4.2).

Lemma 4.3. In the setting of Definition 4.2 suppose that $g:\left(S^{\prime}, Y\right) \rightarrow(R, X)$ is a partial section of $f:(R, X) \rightarrow(S, Y)$. As in Lemma 4.1 we denote by $W_{1}(R)$ the subgroup of $W(R)$ generated by $R_{1}=\{0\} \cup\{\alpha \in R: f(a) \neq 0\}$ and by $\phi_{1}: W_{1}(R) \rightarrow W(S)$ the unique group epimorphism satisfying $\phi_{1}\left(s_{\alpha}\right)=s_{f(\alpha)}$.

Then there exists a unique group monomorphism $\psi: W\left(S^{\prime}\right) \rightarrow W_{1}(R)$ such that $\psi\left(s_{\xi}\right)=s_{g(\xi)}$ for all $\xi \in S^{\prime}$. In particular, $\phi_{1} \circ \psi=\operatorname{Id}_{W\left(S^{\prime}\right)}$.

Proof. Since $g: S^{\prime} \rightarrow R$ is a morphism of reflection systems, Lemma 4.1 (c) shows that $R^{\prime}:=g\left(S^{\prime}\right)$ is a subsystem of $R$ in the subspace $X^{\prime}:=\operatorname{Span}\left(R^{\prime}\right)=$ $g(Y)$ of $X$. Clearly, $f^{\prime}:=f \mid X^{\prime}:\left(R^{\prime}, X^{\prime}\right) \rightarrow\left(S^{\prime}, Y\right)$ is an isomorphism of pre-reflection systems with inverse $g$. Consider the subgroup $H$ of $W(R)$ generated by all $s_{\alpha}, 0 \neq \alpha \in R^{\prime}$. Then $H$ stabilizes $X^{\prime}$ and the restriction map res : $H \rightarrow \mathrm{GL}\left(X^{\prime}\right)$ maps $H$ onto $W\left(R^{\prime}\right)$. Since $f(\alpha) \neq 0$ for $0 \neq \alpha \in R^{\prime}$, we have $H \subset W_{1}(R)$ and thus $H$ fixes $Z:=\operatorname{Ker}(f)$ pointwise, by Lemma 4.1 (b). Now $X=X^{\prime} \oplus Z$ shows that res : $H \rightarrow W\left(R^{\prime}\right)$ is an isomorphism. Since $W\left(f^{\prime}\right): W\left(R^{\prime}\right) \rightarrow W\left(S^{\prime}\right)$ is an isomorphism, there exists a unique homomorphism $\psi$ making the diagram

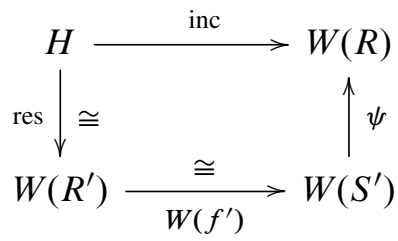

commutative, from which the assertions follow easily. 
Proposition 4.4. Let $f:(R, X) \rightarrow(S, Y)$ be a morphism of reflection systems with $f(R)=S$. We use the notation of Lemma 4.1.

(a) Let $B \subset S$ be a vector space basis of $Y$ and let $S_{B} \subset S$ be the subsystem of $S$ generated by $B$ as in (2.14). Then there exists a partial section $g:\left(S_{B}, Y\right) \rightarrow$ $(R, X)$ of $f$ over $S_{B}$.

(b) Let $S^{\prime} \subset S$ be a subsystem spanning $Y$ and let $g:\left(S^{\prime}, Y\right) \rightarrow(R, X)$ be a partial section of $f$. Suppose in addition that $\mathbb{K}^{\times} \xi \cap\left(S^{\prime}\right)^{\mathrm{re}} \neq \emptyset$ for every $\xi \in S^{\mathrm{re}}$. Then $W(S)=W\left(S^{\prime}\right)$ and the group monomorphism $\psi: W(S) \rightarrow W_{1}(R)$ constructed in Lemma 4.3 splits the exact sequence (4.2), hence $W_{1}(R)=$ $\psi(W(S)) \ltimes V$. For $x \in S$ and $z \in Z$ define endomorphisms $v_{\xi, z}$ of $X$ by

$$
v_{\xi, z}(x)=x-\left\langle f(x), \xi^{\vee}\right\rangle z .
$$

Then the kernel $V$ of $\phi_{1}$ is generated by the maps

$$
v_{f(w \alpha), \alpha-(g \circ f)(\alpha)}, \quad w \in W_{1}(R) \text { and } \alpha \in R_{1} .
$$

The Weyl group $W(R)$ acts on $V$ by

$$
w v_{\xi, z} w^{-1}=v_{\phi(w)(\xi), w(z)} \quad(w \in W(R)) .
$$

Proof. (a) Choose a pre-image $\beta \in R$ for every $\eta \in B$, and define $g: Y \rightarrow X$ to be the $\mathbb{K}$-linear map sending $\eta$ to $\beta$, for all $\eta \in B$. Since $B$ is a vector space basis of $Y$, we have $f \circ g=\operatorname{Id}_{Y}$. We show that $g\left(S_{B}\right) \subset R$. From (2.14) it follows that for every $0 \neq \xi \in S_{B}$ there exist $\eta_{0}, \ldots, \eta_{n} \in B$ such that $\xi=$ $s_{\eta_{n}} \cdots s_{\eta_{1}}\left(\eta_{0}\right)$. Put $\beta_{i}:=g\left(\eta_{i}\right)$ and $\alpha:=s_{\beta_{n}} \cdots s_{\beta_{1}}\left(\beta_{0}\right)$. Then $\alpha \in R$ because $R$ is a reflection system, and $f(\alpha)=\xi$ because $f$ is a morphism of reflection systems. Moreover, $\alpha \in \operatorname{Span}\left\{\beta_{0}, \ldots, \beta_{n}\right\} \subset g(Y)$. Now $f: g(Y) \rightarrow Y$ is a vector space isomorphism, and $f(\alpha-g(\xi))=\xi-\xi=0$, which implies $\alpha=g(\xi) \in R$, as asserted. To prove that $g$ is a morphism of reflection systems, note that $s_{g(\xi)}(g(\eta))$ is a linear combination of $g(\xi)$ and $g(\eta)$ and hence contained in $g(Y)$. Since $f: g(Y) \rightarrow Y$ is a vector space isomorphism, it suffices to show that $f\left(g\left(s_{\xi}(\eta)\right)\right)=f\left(s_{g(\xi)}(g(\eta))\right)$. But this follows from $f \circ g=\operatorname{Id}_{Y}$ and the fact that $f$ is a morphism of reflection systems.

(b) By assumption and (ReS3) we have $s_{\xi} \in W\left(S^{\prime}\right)$ for every $\xi \in S^{\text {re }}$. Hence $W(S)=W\left(S^{\prime}\right)$, and then $\phi_{1} \circ \psi=\operatorname{Id}_{W(S)}$ by Lemma 4.3. Thus $\psi$ splits the exact sequence (4.2), and $W_{1}(R)$ is the semidirect product as indicated.

We prove formula (4.4). Note that $v_{\xi, z}=$ Id for $\xi \in S^{\mathrm{im}}$ because $\xi^{\vee}=0$. Since then also $\phi(w)(\xi) \in S^{\text {im }}$, it follows that (4.4) holds for $\xi \in S^{\text {im }}$. Let now $\xi \in S^{\text {re }}$, say $\xi=f(\alpha)$ for some $\alpha \in R_{1}^{\text {re }}$. It is sufficient to verify (4.4) for $w=s_{\beta}, \beta \in R^{\text {re }}$. 
Note that $s_{\beta} \alpha \in R_{1}$ and hence $\eta=f\left(s_{\beta} \alpha\right)=\phi\left(s_{\beta}\right) \xi \in S^{\text {re }}$. Using (2.8) several times, we get $\left\langle f(x), \xi^{\vee}\right\rangle=\left\langle x, \alpha^{\vee}\right\rangle=\left\langle s_{\beta} x,\left(s_{\beta} \alpha\right)^{\vee}\right\rangle=\left\langle f\left(s_{\beta} x\right), \eta^{\vee}\right\rangle$ and thus

$$
\begin{aligned}
s_{\beta} v_{\xi, z}(x) & =s_{\beta}(x)-\left\langle f(x), \xi^{\vee}\right\rangle s_{\beta}(z)=s_{\beta}(x)-\left\langle f s_{\beta}(x), \eta^{\vee}\right\rangle s_{\beta}(z) \\
& =v_{\eta, s_{\beta}(z)} s_{\beta}(x) .
\end{aligned}
$$

This establishes (4.4).

Let now $\alpha \in R_{1}^{\text {re }}$ and put $\xi=f(\alpha)$. By assumption, there exists $\beta \in g\left(S^{\prime}\right)$ such that $\xi=d f(\beta)$ for some $d \in \mathbb{K}^{\times}$. Note that $\alpha-(g \circ f)(\alpha)=\alpha-d \beta \in \operatorname{Ker} f$ and $\beta \in R_{1}^{\text {re }}$, whence also $s_{\alpha} \beta \in R_{1}$. Using again (2.8), we get

$$
\begin{aligned}
\left\langle s_{\alpha}(x), \beta^{\vee}\right\rangle & =\left\langle x,\left(s_{\alpha} \beta\right)^{\vee}\right\rangle=\left\langle f(x),\left(f\left(s_{\alpha} \beta\right)\right)^{\vee}\right\rangle=\left\langle f(x),\left(s_{\xi} f(\beta)\right)^{\vee}\right\rangle \\
& =-\left\langle f(x), f(\beta)^{\vee}\right\rangle=-\left\langle f(x),\left(d^{-1} \xi\right)^{\vee}\right\rangle=-\left\langle f(x), \xi^{\vee}\right\rangle d,
\end{aligned}
$$

and therefore

$$
\begin{aligned}
s_{\beta} s_{\alpha}(x) & =x-\left\langle x, \alpha^{\vee}\right\rangle-\left\langle x,\left(s_{\alpha} \beta\right)^{\vee}\right\rangle \beta \\
& =x-\left\langle f(x), \xi^{\vee}\right\rangle \alpha+\left\langle f(x), \xi^{\vee}\right\rangle d \beta \\
& =v_{\xi, \alpha-(g \circ f)(\alpha)}(x) .
\end{aligned}
$$

Note that $\phi\left(s_{\alpha}\right)=s_{f(\alpha)}=s_{f(\beta)}=\phi\left(s_{\beta}\right)$ implies $v_{\xi, \alpha-(g \circ f)(\alpha)} \in V$ (where $\xi=f(\alpha)$ as above). Since $V$ is a normal subgroup, we also have

$$
w v_{f(\alpha), \alpha-(g \circ f)(a)} w^{-1}=v_{f(w \alpha), \alpha-(g \circ f)(\alpha)} \in V
$$

for any $w \in W_{1}(R)$. Finally, let $w \in W_{1}(R)$ be an arbitrary element, say $w=$ $s_{\alpha_{1}} \cdots s_{\alpha_{n}}$ with $\alpha_{i} \in R_{1}^{\text {re }}$. Choose $\beta_{i} \in g\left(S^{\prime}\right)$ as in the proof above, so that $s_{\beta_{i}} s_{\alpha_{i}}=v_{i} \in V$. Put $x_{i}=s_{\beta_{1}} \cdots s_{\beta_{i-1}} \in W_{1}(R)$. Then

$$
w=\left(s_{\beta_{n}} \cdots s_{\beta_{1}}\right)\left(x_{n} v_{n} x_{n}^{-1}\right) \cdots\left(x_{i} v_{i} x_{i}^{-1}\right) \cdots\left(x_{2} v_{2} x_{2}^{-1}\right) v_{1} \text {. }
$$

Since $s_{\beta_{n}} \cdots s_{\beta_{1}} \in \psi(W(S))$ and all factors $x_{i} v_{i} x_{i}^{-1} \in V$ are of type (4.3), it follows that $V$ is generated by these maps.

Remark. Let $S$ be an integral reflection system. Observe that $S_{\text {ind }}$ satisfies the condition by (c), and hence the structure of $W(R)$ is described in (c) whenever $f: R \rightarrow S$ has a partial section over $S_{\text {ind }}$. By (a), this is so if there exists a vector space basis $B \subset S$ with $S_{\text {ind }} \subset S_{B}$. Such a basis $B$ exists in the following cases:

(i) $S$ is a locally finite root system (Lemma 5.1),

(ii) $S$ is the set of roots associated to an integral root basis in the sense of Hée (2.10),

(iii) $S$ is the set of roots associated with root data in the sense of Moody-Pianzola (\$2.12(c)), for example $S$ is the set of roots of a Kac-Moody algebra. 


\subsection{Separated morphisms and extensions}

We call a morphism $f: R \rightarrow S$ of pre-reflection systems separated if it keeps reflective and imaginary roots separate, i.e., if it maps reflective (imaginary) roots of $R$ to reflective (imaginary) roots of $S$. As $f\left(R^{\mathrm{im}}\right) \subset S^{\mathrm{im}}$ and $f\left(R^{\mathrm{re}}\right) \subset S^{\text {re }} \cup\{0\}$ always holds by (2.5), we see that

$$
\begin{aligned}
f \text { is separated } & \Longleftrightarrow f\left(R^{\mathrm{re}}\right) \subset S^{\text {re }} \\
& \Longleftrightarrow f(\alpha) \neq 0 \text { for all } \alpha \in R^{\text {re }} .
\end{aligned}
$$

We list three more equivalent conditions for $f$ to be separated:

$$
\begin{aligned}
& \left\langle x, \alpha^{\vee}\right\rangle=\left\langle f(x), f(\alpha)^{\vee}\right\rangle \text { for all } x \in X, \alpha \in R, \\
& f^{-1}\left(\bigcap_{\xi \in f(R)} \operatorname{Ker}\left(\xi^{\vee}\right)\right)=\bigcap_{\alpha \in R} \operatorname{Ker}\left(\alpha^{\vee}\right),
\end{aligned}
$$

and, if $S$ is nondegenerate and $f(R)=S$,

$$
\operatorname{Ker}(f)=\bigcap_{\alpha \in R} \operatorname{Ker}\left(\alpha^{\vee}\right)
$$

Indeed, let $f$ be separated. Then (4.8) holds trivially for $\alpha \in R^{\mathrm{im}}$, and it holds for $\alpha \in R^{\text {re }}$ by (2.8). Now suppose we have (4.8). Then $\left\langle f(x), \xi^{\vee}\right\rangle=0$ for all $\xi \in$ $f(R)$ if and only if $\left\langle x, \alpha^{\vee}\right\rangle=0$ for all $\alpha \in R$, showing that (4.9) holds. Suppose that (4.9) holds, and assume, aiming for a contradiction, that there exists $\beta \in R^{\text {re }}$ with $f(\beta)=0$. Then $f(\beta)$ is annihilated by all $\xi^{\vee}$, whence $\beta \in \bigcap_{\alpha \in R} \operatorname{Ker}\left(\alpha^{\vee}\right)$ by (4.9), which is impossible because $\left\langle\beta, \beta^{\vee}\right\rangle=2$. Finally, (4.10) is immediate from (4.9) because $\bigcap_{\xi \in S} \operatorname{Ker}\left(\xi^{\vee}\right)=\{0\}$ by non-degeneracy.

A morphism $f:(R, X) \rightarrow(S, Y)$ of pre-reflection systems is called an extension if it is separated and satisfies $f(R)=S$. (Strictly speaking, this should be called a separated extension, but since non-separated morphisms with $f(R)=S$ play no role in this paper, we will stay with the simpler terminology.) Thus

$$
f \text { is an extension } \Longleftrightarrow f\left(R^{\mathrm{re}}\right)=S^{\mathrm{re}} \text { and } f\left(R^{\mathrm{im}}\right)=S^{\mathrm{im}} .
$$

By the usual abuse of terminology, we will say that $R$ is an extension of $S$ if there exists an extension map $f: R \rightarrow S$.

Let us point out that, if $R$ is an extension of a nondegenerate $S$ then $S$ is unique up to a unique isomorphism. Indeed, let $f: R \rightarrow S$ and $\tilde{f}: R \rightarrow \tilde{S}$ be extensions of $R$ of nondegenerate reflection systems $S$ and $\tilde{S}$ respectively. Then 
$\operatorname{Ker}(f)=\operatorname{Ker}(\tilde{f})$ by (4.10). From $f(R)=S$ and $\tilde{f}(R)=\tilde{S}$ it follows that $f: X \rightarrow Y$ and $\tilde{f}: X \rightarrow \tilde{Y}$ are surjective. Hence there exists a unique vector space isomorphism $h: Y \rightarrow \tilde{Y}$ such that $h \circ f=\tilde{f}$, and it is easily checked that $h$ is in fact an isomorphism of pre-reflection systems. Therefore, if $R$ is an extension of a nondegenerate pre-reflection system $S$, we are justified in calling $S$ the quotient pre-reflection system and the extension map $f: R \rightarrow S$ the canonical projection.

Remark. If $R$ is nondegenerate it follows from condition (4.9) that every extension $f: R \rightarrow S$ is injective, hence an isomorphism. In particular, a locally finite root system $R$ does not arise as a non-trivial extension of a pre-reflection system $S$. On the other hand, a locally finite root system $S$ does have many interesting extensions $R$, which we will study in the next section.

Lemma 4.5. Let $f:(R, X) \rightarrow(S, Y)$ be an extension of pre-reflection systems.

(a) $R$ is integral or coherent if and only if $S$ is, respectively, integral or coherent.

(b) If $S$ is saturated then so is $R$.

(c) $f$ maps a root string $\mathbb{S}(\beta, \alpha), \beta \in R, \alpha \in R^{\text {re }}$, injectively to $\mathbb{S}(f(\beta), f(\alpha))$.

(d) Suppose $R$ or, equivalently, $S$ is coherent.

(i) The map $C \mapsto f(C)$ is a bijection between the set of connected components of $\operatorname{Re}(R)$ and of $\operatorname{Re}(S)$.

(ii) If in addition $S$ is nondegenerate then

$$
\begin{aligned}
\operatorname{Re}(R) \text { is connected } & \Longleftrightarrow \operatorname{Re}(S) \text { is connected } \\
& \Longleftrightarrow \operatorname{Re}(S) \text { is indecomposable. }
\end{aligned}
$$

(e) Let $S$ be a partial root system. Then $R$ is integral and satisfies the axioms (PRS1) and (PRS3) of a partial root system. Moreover, all root strings $\mathbb{S}(\beta, \alpha)$ $\left(\beta \in R, \alpha \in R^{\mathrm{re}}\right)$ are finite, so $R$ is a partial root system if and only if all these root strings are unbroken.

Proof. (a) is immediate from (4.8) and $f\left(R^{\mathrm{re}}\right)=S^{\mathrm{re}}, f\left(R^{\mathrm{im}}\right)=S^{\mathrm{im}}$. For (b) assume that $\alpha \in R^{\mathrm{re}}, c \in \mathbb{K}^{\times}$and $c \alpha \in R$. Then $\xi=f(\alpha) \in S^{\mathrm{re}}$, and $f(c \alpha)=$ $c \xi \in S$. Since $S$ is saturated we see $c \xi \in S^{\text {re }}$, whence $c \alpha \in R^{\text {re }}$. For (c) we obviously have $f(\mathbb{S}(\beta, \alpha)) \subset \mathbb{S}(f(\beta), f(\alpha))$, and $f(\alpha) \in S^{\text {re }}$. If $f(\beta+i \alpha)=$ $f(\beta+j \alpha)$ then $(i-j) f(\alpha)=0$ which forces $i=j$.

(d) By $f\left(R^{\text {re }}\right)=S^{\text {re }}$ and (4.8) the image of any chain connecting $\alpha$ and $\beta$ in $R^{\text {re }}$ is a chain in $S^{\text {re }}$ connecting $f(\alpha)$ and $f(\beta)$ in $S^{\text {re }}$, and conversely any chain 
connecting $f(\alpha)$ and $f(\beta)$ in $S^{\text {re }}$ can be lifted to a chain connecting $\alpha$ and $\beta$ in $R^{\text {re }}$. This proves (d.i). The first equivalence in (4.12) follows from (i) and the second from Lemma 2.1.

For the proof of (e), we know already from (a) that $R$ is integral and from (c) that all root strings $\mathbb{S}(\beta, \alpha), \alpha \in R^{\text {re }}$, are finite. Thus it remains to show that (PRS1) and (PRS3) hold in $R$. To do so, let $\alpha, \beta \in R^{\text {re }}$ and put $\xi=f(\alpha), \eta=f(\beta)$. Then $\left\langle\beta, \alpha^{\vee}\right\rangle=\left\langle\eta, \xi^{\vee}\right\rangle$ by (4.8), and since $\xi, \eta \in S^{\text {re }}$ and (PRS1) holds in $S$, it also holds in $R$. For (PRS3), assume $\alpha+\beta \in R$ and $\left\langle\beta, \alpha^{\vee}\right\rangle \geq 0$. If $\alpha+\beta \in R^{\text {im }}$ then $\xi+\eta=f(\alpha+\beta) \in S^{\text {im }}$, contradicting (PRS3) for $S$.

\subsection{Extension data}

Let $(S, Y)$ be a pre-reflection system, let $S^{\prime}$ be a subsystem of $S$ with $\operatorname{Span}\left(S^{\prime}\right)=$ $Y$ and let $Z$ be a $\mathbb{K}$-vector space. A family $\mathbb{L}=\left(\Lambda_{\xi}\right)_{\xi \in S}$ of nonempty subsets of $Z$ is called an extension datum of type $\left(S, S^{\prime}, Z\right)$ if

(ED1) for all $\xi, \eta \in S$ and all $\lambda \in \Lambda_{\xi}, \mu \in \Lambda_{\eta}$ we have $\mu-\left\langle\eta, \xi^{\vee}\right\rangle \lambda \in \Lambda_{s_{\xi}(\eta)}$,

(ED2) $0 \in \Lambda_{\xi^{\prime}}$ for all $\xi^{\prime} \in S^{\prime}$, and

(ED3) $Z$ is spanned by the union of all $\Lambda_{\xi}, \xi \in S$.

Let $\mathfrak{R}$ be an extension datum. We will derive some immediate consequences of the axioms (ED1)-(ED3). For $\xi=\eta \in S^{\text {re }}$ and $\lambda \in \Lambda_{\xi}$, (ED1) implies $-\lambda=\lambda-2 \lambda \in \Lambda_{-\xi}$, hence

$$
\Lambda_{-\xi}=-\Lambda_{\xi} \quad \text { for all } \xi \in S^{\mathrm{re}} .
$$

Again from (ED1) for $\eta=\xi \in S^{\text {re }}$ we then get $\Lambda_{\xi}-2 \Lambda_{\xi}:=\{\mu-2 \lambda: \mu, \lambda \in$ $\left.\Lambda_{\xi}\right\} \subset \Lambda_{-\xi}=-\Lambda_{\xi}$, and therefore

$$
2 \Lambda_{\xi}-\Lambda_{\xi} \subset \Lambda_{\xi} \text { for all } \xi \in S^{\text {re }} .
$$

Also, from (ED1) and (ED2) we obtain $\Lambda_{\eta} \subset \Lambda_{s_{\xi^{\prime}}(\eta)}$ for all $\eta \in S$ and $\xi^{\prime} \in S^{\prime}$, whence $\Lambda_{\eta}=\Lambda_{s_{\xi^{\prime}}(\eta)}$ and then

$$
\Lambda_{\eta}=\Lambda_{w^{\prime}(\eta)} \quad \text { for all } \eta \in S \text { and } w^{\prime} \in W_{S^{\prime}},
$$

where $W_{S^{\prime}}:=W_{S^{\prime}}(S) \subset W(S)$ is the subgroup generated by all $s_{\xi}, \xi \in S^{\prime}$, cf. §2.5. Finally, (ED1) and (4.15) for $w^{\prime}=s_{\xi^{\prime}}$ yield

$$
\begin{aligned}
& \Lambda_{\eta}-\left\langle\eta, \xi^{\vee}\right\rangle \Lambda_{\xi^{\prime}} \subset \Lambda_{\eta} \text { and } \\
& \Lambda_{\xi^{\prime}}=\Lambda_{-\xi^{\prime}} \text { for } \xi^{\prime} \in S^{\prime}, \eta \in S .
\end{aligned}
$$


The condition (ED3) only serves to determine $Z$ and can always be achieved by replacing $Z$ with the span of the $\Lambda_{\xi}, \xi \in S$. It will sometimes be convenient to leave $Z$ unspecified, in which case we will employ the terminology extension datum of type $\left(S, S^{\prime}\right)$ for a family $\left(\Lambda_{\xi}\right)_{\xi \in S}$ of subsets of a some vector space satisfying (ED1) and (ED2).

The only condition on $\Lambda_{0}$ is

$$
0 \in \Lambda_{0} .
$$

Moreover, $\Lambda_{0}$ is related to the other $\Lambda_{\xi}, \xi \neq 0$, only by axiom (ED3). It follows that it is always possible to modify a given extension datum $\mathfrak{L}=\left(\Lambda_{\xi}\right)_{\xi \in S}$ of type $\left(S, S^{\prime}\right)$ by replacing $\Lambda_{0}$ by any other set containing 0 . The modified extension datum will again be of type $\left(S, S^{\prime}\right)$ but, because of (ED3), not necessarily of type $\left(S, S^{\prime}, Z\right)$ if $\mathbb{Z}$ was of this type. In particular, $\Lambda_{0}=\{0\}$ is always a possible choice. An extension datum $\mathfrak{R}$ with $\Lambda_{0}=\{0\}$ will be called of minimal type. For the purpose of classification, it is natural to assume $\mathfrak{L}$ of minimal type. This is in fact how extension data have appeared in the literature, see $\$ 4.3$. On the other hand, the choice of $\Lambda_{0}$ influences in an essential way the properties of the associated reflection system $\mathcal{E}\left(S, S^{\prime}, Z\right)$, see for example Corollary 5.2 for the case of affine reflection systems.

\subsection{Examples}

(a) Extension data occur in the theory of algebraic groups over local fields. Namely, let $S$ be a finite root system and let $\phi=\left(\phi_{\xi}\right)_{\xi \in S^{\times}}$be a special valuation of a root datum, as defined in [15, (6.2)]. Then, in the notation of [15, (6.2.2)], the family $\Lambda_{\xi}=\Gamma_{\xi}^{\prime} \subset \mathbb{R}$ for $\xi \in S^{\times}$and $\Lambda_{0}=\{0\}$, is an extension datum of type $\left(S, S_{\text {ind }}, \mathbb{R}\right)$. Indeed, condition (ED1) follows from [15, (6.2.14.1)] and $\Gamma_{\xi}^{\prime} \subset \Gamma_{\xi}$ in the notation of [15], while (ED2) holds by definition of a special valuation in $[15,(6.2 .13)]$.

(b) Let $S$ be an integral reflection system. Then our definition of an extension datum makes sense for any abelian group $Z$ instead of a vector space. Since in this paper we have no use of this generality, we have restricted ourselves to the case of a vector space $Z$. But we wish to point out that for $S$ a finite irreducible root system and $Z$ an abelian group, extension data of minimal type $\left(S, S_{\text {ind }}, Z\right)$ were defined by Yoshii in [48], as "root systems of type $S$ extended by $Z$ ". The paper [48] contains a classification of extension data for finite irreducible root systems [48, Theorem 2.4]. It is an easy exercise, which we will leave to the reader, to extend this to the case of an irreducible locally finite root system. For a detailed study of extension data of minimal type $\left(S, S_{\text {ind }}, \mathbb{R}^{n}\right)$, where $S$ is a finite 
irreducible root system and all $\Lambda_{\xi}$ are contained in a lattice of $\mathbb{R}^{n}$, the reader is referred to [1, Chapter II] and [5].

Theorem 4.6. Let $(S, Y)$ be a pre-reflection system.

(a) Let $\mathfrak{L}=\left(\Lambda_{\xi}\right)_{\xi \in S}$ be an extension datum of type $\left(S, S^{\prime}, Z\right)$. Put $X:=Y \oplus Z$, denote by $\pi: X \rightarrow Y$ the projection with kernel $Z$, and define

$$
\begin{aligned}
R & :=\bigcup_{\xi \in S} \xi \oplus \Lambda_{\xi} \subset X, \quad \text { and } \\
s_{\alpha}(x) & :=s_{\xi}(y) \oplus\left(z-\left\langle y, \xi^{\vee}\right\rangle \lambda\right),
\end{aligned}
$$

for all $\alpha=\xi \oplus \lambda \in \xi \oplus \Lambda_{\xi} \subset R$ and all $x=y \oplus z \in X$. Then $R$ is a prereflection system in $X$, denoted $\mathcal{E}=\mathcal{E}\left(S, S^{\prime}, \mathfrak{L}\right)$. Moreover, $\pi:(R, X) \rightarrow$ $(S, Y)$ is an extension of pre-reflection systems, and the canonical injection $\iota: Y \rightarrow X$ is a partial section of $\pi$ over $S^{\prime}$.

(b) Conversely, let $f:(R, X) \rightarrow(S, Y)$ be an extension and let $g: S^{\prime} \rightarrow R$ be a partial section of $f$, cf. Definition 4.2. For every $\xi \in S$ define $R_{\xi} \subset R$ and $\Lambda_{\xi} \subset Z:=\operatorname{Ker}(f)$ by

$$
R_{\xi}=R \cap f^{-1}(\xi)=g(\xi) \oplus \Lambda_{\xi}
$$

Then $\mathfrak{R}=\left(\Lambda_{\xi}\right)_{\xi \in S}$ is an extension datum of type $\left(S, S^{\prime}, Z\right)$, and the vector space isomorphism $\phi: Y \oplus Z \cong X$ sending $y \oplus z$ to $g(y) \oplus z$ is an isomorphism $\mathcal{E}\left(S, S^{\prime}, \mathfrak{L}\right) \cong R$ of pre-reflection systems making the following diagram commutative:

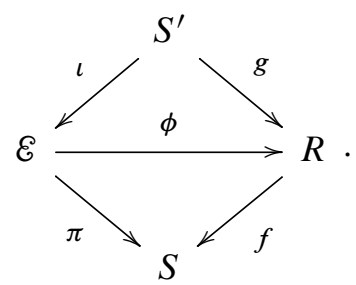

(c) In the setting of (b), the following are equivalent for $g^{\prime} \in \operatorname{Hom}_{\mathbb{K}}(Y, X)$ :

(i) $g^{\prime}: S^{\prime} \rightarrow R$ is another section of $f$,

(ii) there exists $\phi \in \operatorname{Hom}_{\mathbb{K}}(Y, Z)$ such that $g^{\prime}=g+\phi$ and $\phi\left(\xi^{\prime}\right) \in \Lambda_{\xi^{\prime}}$ for all $\xi^{\prime} \in S^{\prime}$. 
Proof. (a) We have $0 \in R$ by (ED2). To show that $R$ spans $X$ let $x=y \oplus z \in X$ with $y \in Y$ and $z \in Z$. Because of (ED3) there exist $\xi_{i} \in S, \lambda_{i} \in \Lambda_{\xi_{i}}$ and $t_{i} \in \mathbb{K}$ such that $z=\sum_{i} t_{i} \lambda_{i}$. Also, since $\operatorname{Span}\left(S^{\prime}\right)=Y$ there exist $\xi_{j}^{\prime} \in S^{\prime}$ and $t_{j}^{\prime} \in \mathbb{K}$ such that $y-\sum_{i} t_{i} \xi_{i}=\sum_{j} t_{j}^{\prime} \xi_{j}^{\prime}$. Then $y \oplus z=\sum_{i} t_{i}\left(\xi_{i} \oplus \lambda_{i}\right)+\sum_{j} t_{j}^{\prime} \xi_{j}^{\prime} \in$ $\operatorname{Span}(R)$ by (ED2) and the definition of $R$.

We now prove that $R$ is a pre-reflection system, by verifying the axioms listed in $\S 2.3$. For $\alpha=\xi \oplus \lambda \in \xi \oplus \Lambda_{\xi}$ define $\alpha^{\vee} \in X^{*}$ by

$$
\left\langle x, \alpha^{\vee}\right\rangle:=\left\langle y, \xi^{\vee}\right\rangle=\left\langle\pi(x), \pi(\alpha)^{\vee}\right\rangle,
$$

for all $x=y \oplus z \in X$. Then (4.20) can be rewritten in the familiar form $s_{\alpha}(x)=$ $x-\left\langle x, \alpha^{\vee}\right\rangle \alpha$. We have $\alpha^{\vee} \neq 0$ if and only if $\xi \in S^{\text {re }}$, in which case $\left\langle\alpha, \alpha^{\vee}\right\rangle=$ $\left\langle\xi, \xi^{\vee}\right\rangle=2$ and $(-\alpha)^{\vee} \neq 0$ proving $(\operatorname{ReS} 1)^{\vee}$. From (4.20) and (ED1) we see that $s_{\alpha}(\beta) \in R$ and $\left(s_{\alpha} \beta\right)^{\vee}=0 \Longleftrightarrow \beta^{\vee}=0$ for all $\alpha, \beta \in R$, whence $(\operatorname{ReS} 2)^{\vee}$ also holds. Thus, $R$ is a pre-reflection system. From (4.20) and $\pi(R)=S$ it is now evident that $\pi: R \rightarrow S$ is a morphism of pre-reflection systems, and (4.23) shows that condition (4.8) holds, so $\pi$ is separated. Thus $\pi$ is an extension of $S$. The canonical injection $\iota$ maps $S^{\prime}$ into $R$ because of (ED2) and is a morphism of reflection systems by (4.20). Since $S^{\prime}$ spans $Y, \iota$ is a partial section of $\pi$ over $S^{\prime}$.

(b) Because $f(R)=S$ we have $R_{\xi} \neq \emptyset$ and hence $\emptyset \neq \Lambda_{\xi} \subset Z$ for all $\xi \in S$. Let $\xi, \eta \in S$ and $\lambda \in \Lambda_{\xi}, \mu \in \Lambda_{\eta}$. Then $\alpha=g(\xi) \oplus \lambda$ and $\beta=g(\eta) \oplus \mu$ belong to $R$ and $f(\alpha)=\xi, f(\beta)=\eta$. We compute

$$
\begin{aligned}
s_{\alpha}(\beta) & =\beta-\left\langle\beta, \alpha^{\vee}\right\rangle \alpha=\beta-\left\langle\eta, \xi^{\vee}\right\rangle \alpha \\
& =\left(g(\eta)-\left\langle\eta, \xi^{\vee}\right\rangle g(\xi)\right) \oplus\left(\mu-\left\langle\eta, \xi^{\vee}\right\rangle \lambda\right) \\
& =g\left(s_{\xi}(\eta)\right) \oplus\left(\mu-\left\langle\eta, \xi^{\vee}\right\rangle \lambda\right) .
\end{aligned}
$$

On the other hand, $f\left(s_{\alpha}(\beta)\right)=s_{\xi}(\eta)$ because $f$ is a morphism of pre-reflection systems, which shows that $s_{\alpha}(\beta) \in R_{s_{\xi}(\eta)}$ and hence that $\mu-\left\langle\eta, \xi^{\vee}\right\rangle \lambda \in \Lambda_{s_{\xi}(\eta)}$. This establishes axiom (ED1) of $\$ 4.2$. Next, (ED2) follows from $g\left(S^{\prime}\right) \subset R$. Finally, (ED3) holds because $R$ spans $X$. Thus $\mathfrak{L}$ is an extension datum of type $\left(S, S^{\prime}, Z\right)$. That $\phi$ is an isomorphism and that the diagram (4.22) commutes, is clear by construction.

(c) It is obvious that (ii) is equivalent to $f \circ g^{\prime}=\operatorname{Id}_{Y}$ and $g^{\prime}\left(S^{\prime}\right) \subset R$. It therefore suffices to prove that $g^{\prime}=g+\phi$ is a morphism of pre-reflection systems if (ii) holds. Because of (2.8) it is enough to show $\left\langle y, \xi^{\prime \vee}\right\rangle=\left\langle g^{\prime}(y), g^{\prime}\left(\xi^{\prime}\right)^{\vee}\right\rangle$ for $y \in Y$ and $\xi^{\prime} \in S^{\prime}$. But this follows from (4.8) for the extension $f$ and $x=g^{\prime}(y)$ :

$$
\left\langle g^{\prime}(y),\left(g^{\prime}\left(\xi^{\prime}\right)\right)^{\vee}\right\rangle=\left\langle\left(f \circ g^{\prime}\right)(y),\left(f \circ g^{\prime}\right)\left(\xi^{\prime}\right)^{\vee}\right\rangle=\left\langle y, \xi^{\prime \vee}\right\rangle .
$$


Corollary 4.7. Let $(S, Y)$ be a pre-reflection system and let $\mathfrak{R}=\left(\Lambda_{\xi}\right)_{\xi \in S}$ be an extension datum of type $\left(S, S^{\prime}, Z\right)$. Let $R=\mathcal{E}\left(S, S^{\prime}, Z\right)$ be the pre-reflection system defined in Theorem 4.6 (a).

(a) $R$ is reduced if and only if, for all $\xi \in S$,

$$
\xi, c \xi \in S^{\times} \text {for } c \in \mathbb{K}^{\times} \backslash\{ \pm 1\} \quad \Longrightarrow \quad \Lambda_{c \xi} \cap c \Lambda_{\xi}=\emptyset .
$$

(b) $R$ is symmetric if and only if $S$ is symmetric and $\Lambda_{-\xi}=-\Lambda_{\xi}$ for all $\xi \in S^{\mathrm{im}}$.

(c) $R$ is a reflection system if and only if $S$ is a reflection system.

(d) Suppose $S$ is a partial root system. Then $R$ is a partial root system if and only if

$$
\eta \in S, \xi \in S^{\mathrm{re}} \text { and }\left\langle\eta, \xi^{\vee}\right\rangle \geq 2 \Longrightarrow \Lambda_{\eta}-\Lambda_{\xi} \subset \Lambda_{\eta-\xi} .
$$

Proof. (a) and (b) are immediate from the definitions. In (c) it is obvious from (4.23) that axiom (ReS3) $)^{\vee}$ of $\S 2.3$ holds in $R$ if and only if it holds in $S$. Also, if $(\operatorname{ReS} 4)^{\vee}$ holds in $R$, then it also holds in $S$ because of (2.8). That the converse is also true follows from the following calculation

$$
\left\langle x, s_{\alpha}(\beta)^{\vee}\right\rangle=\left\langle y, s_{\xi}(\eta)^{\vee}\right\rangle=\left\langle y, \eta^{\vee}-\left\langle\xi, \eta^{\vee}\right\rangle \xi^{\vee}\right\rangle=\left\langle x, \beta^{\vee}-\left\langle\alpha, \beta^{\vee}\right\rangle \alpha^{\vee}\right\rangle
$$

for $x=y \oplus z \in X, \alpha=\xi \oplus \lambda$ and $\beta=\eta \oplus \mu$. For (d) it suffices by Lemma 4.5 (e) to evaluate the condition that all root strings $\mathbb{S}(\beta, \alpha)$ for $\beta \in R$ and $\alpha \in R^{\text {re }}$ are unbroken. Because of Lemma 3.1 (c) and $-\alpha^{\vee}=(-\alpha)^{\vee}$, this holds if and only if $\beta-\alpha \in R$ whenever $\alpha, \beta$ as above satisfy $\left\langle\beta, \alpha^{\vee}\right\rangle>0$. Write $\alpha=\xi \oplus \lambda$ and $\beta=\eta \oplus \mu$ as usual, and suppose $\left\langle\beta, \alpha^{\vee}\right\rangle=\left\langle\eta, \xi^{\vee}\right\rangle>0$. Then $\eta-\xi \in S$ since $S$ has unbroken root strings, and hence $\beta-\alpha \in R$ if and only if $\lambda-\mu \in \Lambda_{\eta-\xi}$, i.e., $\Lambda_{\eta}-\Lambda_{\xi} \subset \Lambda_{\eta-\xi}$. By (ED1) this always holds for $\left\langle\eta, \xi^{\vee}\right\rangle=1$. Thus all root strings of $R$ are unbroken if and only if (4.26) is satisfied.

\section{Affine reflection systems}

Lemma 5.1. Let $(S, Y)$ be a locally finite root system with Weyl group $W(S)$. Then there exists an integral basis $B$ of $S$ such that $S_{\text {ind }}^{\times}=W(S) \cdot B$ and $W(S)$ is generated by all $s_{\alpha}, \alpha \in B$; hence, the reflection subsystem of $S$ generated by $B$ is $S_{\text {ind. }}$.

Recall $[26,6.1]$ that, by definition, $B \subset S$ is an integral basis if $B$ is linearly independent over $\mathbb{K}$ and every $\xi \in S$ is a $\mathbb{Z}$-linear combination of $B$. Hence $B$ is in particular a basis of the $\mathbb{K}$-vector space $Y$. 
Proof. Let $(S, Y)=\bigsqcup_{i \in I}\left(S_{i}, Y_{i}\right)$ be the decomposition of $(S, Y)$ into its irreducible components, cf. $\$ 2.12$ (a). Then $W(S)=\bigoplus_{i \in I} W\left(S_{i}\right)$ is the restricted direct product of the Weyl groups $W\left(S_{i}\right)$ of the irreducible locally finite root system $\left(S_{i}, Y_{i}\right)$, see $[26,5.2 .2]$ or Lemma $2.1, \S 2.4$. We may therefore assume that $(S, Y)$ is irreducible. If $S$ is finite, we may take for $B$ a root basis, see e.g. [14, VI, $\S 1.5$, Theorem 2 and Proposition 15]. If $S$ is infinite, root bases need not exist [26, p. 49], so we revert to a case-by-case proof. By [26, Theorem 8.4], $S$ is isomorphic to $\dot{\mathrm{A}}_{I}, \mathrm{D}_{I}, \mathrm{~B}_{I}, \mathrm{C}_{I}$ or $\mathrm{BC}_{I}$ where $I$ is an infinite set. It suffices to find an integral basis $B$ with the following property: Denoting the subgroup of $W(S)=W\left(S_{\text {ind }}\right)$ generated by all $s_{\beta}, \beta \in B$ by $W^{\prime}$, every $\alpha \in S_{\text {ind }}^{\times}$is of the form

$$
\alpha=w(\beta) \in W^{\prime} \cdot B
$$

for some $w \in W^{\prime}$ and some $\beta \in B$. Indeed, then $s_{\alpha}=w s_{\beta} w^{-1} \in W^{\prime}$ which implies $W(S)=W^{\prime}$. Furthermore, since $\alpha=w(\beta)$ implies $-\alpha=w s_{\beta}(\beta) \in$ $W^{\prime} \cdot B$, it is enough to prove (*) for $\alpha$ in a subset $P$ of $S_{\text {ind }}^{\times}$with $P \cup(-P)=S_{\text {ind }}^{\times}$. Also note that, if $(*)$ holds for $\alpha$, it will hold for all $s_{\alpha}(\gamma), \gamma \in B$, as well, because $s_{\alpha}(\gamma)=w s_{\beta} w^{-1}(\gamma) \in W^{\prime} \cdot B$.

We now fix an element of $I$, denoted by 0 , and put $J:=I \backslash\{0\}$ for simplicity.

Case $\dot{\mathrm{A}}_{I}$. Let $B=\left\{\varepsilon_{0}-\varepsilon_{j}: j \in J\right\}$. Evidently, $B$ is an integral basis. For distinct $i, j \in J$ we have $\varepsilon_{i}-\varepsilon_{j}=s_{\varepsilon_{0}-\varepsilon_{i}}\left(\varepsilon_{0}-\varepsilon_{j}\right)$, cf. formula [26, 9.5.4]. Thus (*) holds for $P=B \cup\left\{\varepsilon_{i}-\varepsilon_{j}: i, j \in J, i \neq j\right\}$.

Case $\mathrm{D}_{I}$. Here we fix another element $1 \in J$ and put $B=\left\{\varepsilon_{0}+\varepsilon_{j}: j \in J\right\} \cup$ $\left\{\varepsilon_{0}-\varepsilon_{1}\right\}$. Again, it is easy to see that $B$ is an integral basis. For distinct $i, j \in J$ we have $s_{\varepsilon_{0}+\varepsilon_{i}}\left(\varepsilon_{0}+\varepsilon_{j}\right)=-\varepsilon_{i}+\varepsilon_{j}$, cf. [26, 9.5.5], and furthermore $\varepsilon_{0}-\varepsilon_{i}=$ $s_{\varepsilon_{1}-\varepsilon_{i}}\left(\varepsilon_{0}-\varepsilon_{1}\right)=s_{\varepsilon_{0}+\varepsilon_{i}} s_{\varepsilon_{0}+\varepsilon_{1}} s_{\varepsilon_{0}+\varepsilon_{i}}\left(\varepsilon_{0}-\varepsilon_{1}\right)$ as well as $\varepsilon_{i}+\varepsilon_{j}=s_{\varepsilon_{0}-\varepsilon_{i}}\left(\varepsilon_{0}+\varepsilon_{j}\right)$, which proves $(*)$ for all $\alpha$ in

$$
P=\left\{\varepsilon_{i} \pm \varepsilon_{j}: i, j \in J, i \neq j\right\} \cup\left\{\varepsilon_{0}-\varepsilon_{j}: j \in J\right\} .
$$

Cases $\mathrm{B}_{I}, \mathrm{C}_{I}$ and $\mathrm{BC}_{I}$. Let $m=1$ if $S=\mathrm{B}_{I}$ or $S=\mathrm{BC}_{I}$ (in these cases, $\left.S_{\text {ind }}=\mathrm{B}_{I}\right)$, and $m=2$ if $S=S_{\text {ind }}=\mathrm{C}_{I}$. Then $S_{\text {ind }}=\mathrm{D}_{I} \cup\left\{ \pm m \varepsilon_{i}: i \in I\right\}$ in all cases. We put $B=\left\{m \varepsilon_{0}\right\} \cup\left\{\varepsilon_{0}+\varepsilon_{j}: j \in J\right\}$. It is easily checked that $B$ is an integral basis of $S$. Fix again an element $1 \in J$ and note that $\varepsilon_{0}-\varepsilon_{1}$ satisfies $(*)$, because $s_{m \varepsilon_{0}} s_{\varepsilon_{0}+\varepsilon_{1}}\left(\varepsilon_{0}+\varepsilon_{1}\right)=\varepsilon_{0}-\varepsilon_{1}$. By what we proved in case $\mathrm{D}_{I}$, we therefore know that $(*)$ holds for all $\alpha \in \mathrm{D}_{I} \subset S_{\text {ind }}$, and hence also for all $\alpha=m \varepsilon_{j}=s_{\varepsilon_{0}-\varepsilon_{j}}\left(m \varepsilon_{0}\right)$, where $j \in J$.

Remark. It is possible to give a classification-free proof using the theory of grid bases [32] in 3-graded root systems. 


\subsection{Affine reflection systems}

A reflection system $(R, X)$ is called affine if it is an extension of a locally finite root system $S$, say, $f:(R, X) \rightarrow(S, Y)$. A morphism between affine reflection systems is a morphism of the underlying reflection systems.

For the convenience of the reader we explicitly state the implications of some of our results for affine reflection systems. We have not defined "affine pre-reflection systems" since by Theorem 4.6 and Corollary 4.7 (c) every extension of a locally finite root system is a reflection system.

In the following let $(R, X)$ be an affine reflection system and $f:(R, X) \rightarrow$ $(S, Y)$ an extension where $(S, Y)$ is a locally finite root system. Since locally finite root systems are nondegenerate (Proposition 2.2 and (2.18)), we have $\operatorname{Ker}(f)=$ $\bigcap_{\alpha \in R} \alpha^{\vee}$ by (4.10). Also, as explained in $\S 4.1$, non-degeneracy implies that $S$ is unique up to unique isomorphism. We will call it the quotient root system of $R$ in this context and refer to $f$ as the canonical projection. We put $Z=\operatorname{Ker}(f)$ and then have $Y \cong X / Z$.

(a) By Lemma 5.1 and Proposition 4.4 (a), $f$ has a partial section $g$ over $S_{\text {ind }}$. Let $\mathfrak{Z}=\left(\Lambda_{\xi}\right)_{\xi \in S}$ be the extension datum of type $\left(S, S_{\text {ind }}, Z\right)$ associated to $f$ and $g$ in Theorem 4.6 (b). Then $R$ is isomorphic to the extension $\mathcal{E}\left(S, S_{\text {ind }}, Z\right)$. Thus, up to an isomorphism which depends on the choice of $g$, we may assume that

$$
R=\bigcup_{\xi \in S}\left(\xi \oplus \Lambda_{\xi}\right) \subset X=Y \oplus Z
$$

(b) Since $S^{\times} \subset \mathbb{K}^{\times} S_{\text {ind }}$, the condition in Proposition 4.4(b) is fulfilled and hence this result together with Lemma 4.1 describes the structure of the Weyl group $W(R)$. Realizing $R$ as in (a), we have

$$
W(R)=W_{S} \ltimes V
$$

where

(i) $W_{S}$ is the subgroup of $W(R)$ generated by the reflections $s_{\alpha}, \alpha \in S_{\text {ind }} \subset R$; the restriction map $W_{S} \rightarrow W(S), w \mapsto w \mid Y$, is an isomorphism of $W_{S}$ onto the Weyl group of $W(S)$ of $S$; each $w \in W_{S}$ fixes $Z$ pointwise (Lemma $4.1(b))$.

(ii) $V$ is an abelian normal subgroup of $W(R)$ generated by the transformations $v_{\xi, \lambda}, \xi \in S$ and $\lambda \in \Lambda_{\xi}$; the action of $v_{\xi, \lambda}$ is $x=y \oplus z \mapsto x-\left\langle y, \xi^{\vee}\right\rangle \lambda$. Indeed, by Proposition 4.4 (b), $V$ is generated by the maps $v_{w \xi, \lambda}$ for $w \in$ $W(S)$ and $\lambda \in \Lambda_{\xi}$, but $\Lambda_{\xi}=\Lambda_{w \xi}$ by (4.15). 
(iii) For $w \in W(R)$ we have $w v_{\xi, \lambda} w^{-1}=v_{w(\xi), w(\lambda)}$, and hence in particular $w v_{\xi, \lambda} w^{-1}=v_{w(\xi), \lambda}$ for $w \in W_{S}$

It is a straightforward task which we leave to the reader to write down a presentation of $W(R)$ based on (i)-(iii), cf. [26, 5.12].

(c) By (4.12) and $\$ 2.12(\mathrm{a}), \operatorname{Re}(R)$ is connected if and only if $S$ is an irreducible root system. Also, we point out that if $Y$ is finite-dimensional then $S$ is a finite root system as defined in $[14, \mathrm{VI}]$.

(d) Extension data of locally finite root systems have some special properties, beyond the ones established in $\S 4.2$. Namely, let $S$ be a locally finite root system and let $\mathfrak{L}=\left(\Lambda_{\xi}\right)_{\xi \in S}$ be an extension datum of type $\left(S, S_{\text {ind }}, Z\right)$. Since the Weyl group $W(S)$ is generated by all reflections $s_{\xi}, \xi \in S_{\text {ind }}$, (4.15) implies

$$
\Lambda_{\eta}=\Lambda_{w(\eta)} \quad \text { for all } \eta \in S \text { and } w \in W(S) .
$$

Putting here $w=s_{\eta}$ and using (4.13) yields

$$
\Lambda_{\eta}=\Lambda_{-\eta}=-\Lambda_{\eta} \quad \text { for all } \eta \in S^{\times} .
$$

Also, (5.3) for $w=s_{\xi}$ and (ED1) imply

$$
\Lambda_{\eta}-\left\langle\eta, \xi^{\vee}\right\rangle \Lambda_{\xi} \subset \Lambda_{\eta} \quad \text { for all } \xi, \eta \in S .
$$

In particular, putting here $\xi=\eta \in S^{\times}$we have $\left\langle\xi, \xi^{\vee}\right\rangle=2$, so (5.4) and (5.5) imply

$$
\Lambda_{\xi}+2 \Lambda_{\xi} \subset \Lambda_{\xi} \text { for all } \xi \in S^{\times} .
$$

If $\xi=2 \eta \in S^{\times}$then $\left\langle\eta, \xi^{\vee}\right\rangle=1$ and $\eta \in S_{\text {ind }}$. Hence (5.5) shows $\Lambda_{\eta}-\Lambda_{2 \eta} \subset$ $\Lambda_{\eta}$, and by (ED2) and (5.4) we have

$$
\Lambda_{2 \eta} \subset \Lambda_{\eta} \text { whenever } \eta, 2 \eta \in S,
$$

since in case $\eta=0$ this obviously holds as well.

Corollary 5.2. Let $R$ be an affine reflection system with quotient root system $S$, and let $\mathfrak{L}=\left(\Lambda_{\xi}\right)_{\xi \in S}$ be the extension datum of type $\left(S, S_{\mathrm{ind}}\right)$ associated to $R$ in Theorem 4.6(b). Note $R_{0}=R \cap \operatorname{Ker}(f)=\Lambda_{0}$. Define

$$
\Lambda_{\text {diff }}:=\bigcup_{\xi \in S^{\times}}\left(\Lambda_{\xi}-\Lambda_{\xi}\right)
$$

Then

$$
\mathbb{Z} \Lambda_{\text {diff }}=\Lambda_{\text {diff }}
$$


and we have:

(a) $R$ is symmetric if and only if $R_{0}=-R_{0}$.

(b) $R$ is a partial root system if and only if all root strings $\mathbb{S}(\beta, \alpha)(\beta \in R$, $\alpha \in R^{\mathrm{re}}$ ) are unbroken if and only if $\Lambda_{\mathrm{diff}} \subset R_{0}$.

(c) $R$ is tame in the sense that $R_{0} \subset R^{\mathrm{re}}-R^{\mathrm{re}}$ if and only if $R_{0} \subset \Lambda_{\text {diff. }}$

Proof. For $\xi \in S^{\times}$we have $(2 \mathbb{Z}+1) \Lambda_{\xi} \subset \Lambda_{\xi}$. Indeed, let $\lambda \in \Lambda_{\xi}$ and assume $m \lambda \in \Lambda_{\xi}$ for some $m \in \mathbb{Z}$. Then $-m \lambda \in \Lambda_{\xi}$ by (5.4) and $(m+2) \lambda=m \lambda+2 \lambda \in$ $\Lambda_{\xi}$ by (5.6), so $(2 \mathbb{Z}+1) \Lambda_{\xi} \subset \Lambda_{\xi}$ follows by induction. Moreover, if $\xi \in S_{\text {ind }}^{\times}$ then $0 \in \Lambda_{\xi}$ by (ED2), so $2 \mathbb{Z} \Lambda_{\xi} \subset \Lambda_{\xi}$ follows again by induction, whence

$$
\mathbb{Z} \Lambda_{\xi}=\Lambda_{\xi} \text { for } \xi \in S_{\text {ind }}^{\times} \text {. }
$$

Now (5.9) is a straightforward consequence of (5.7) and (5.10).

(a) follows from Corollary 4.7 (b).

(b) We evaluate the condition (4.26) in our setting. Thus let $\eta, \xi \in S^{\times}$with $\left\langle\eta, \xi^{\vee}\right\rangle \geq 2$. If $\left\langle\xi, \eta^{\vee}\right\rangle=1$ then $\xi-\eta=s_{\xi}(\eta) \in S^{\times}$and (4.26) follows from (ED1) and (4.13). If both $\left\langle\xi, \eta^{\vee}\right\rangle>1$ and $\left\langle\eta, \xi^{\vee}\right\rangle>1$ then by known properties of locally finite root systems [26, A.2], we have $\xi=\eta$. Thus, (4.26) is equivalent to $\Lambda_{\text {diff }} \subset \Lambda_{0}=R_{0}$.

(c) Let $\phi \in R_{0}$ and let $\alpha=\xi \oplus \lambda$ and $\beta=\eta \oplus \mu$ be in $R^{\text {re }}$. Then we have $\phi=\alpha-\beta=(\xi-\eta) \oplus(\lambda-\mu) \in R_{0}$ if and only if $\xi=\eta$ and $\phi=\lambda-\mu \in \Lambda_{\xi}-\Lambda_{\xi}$. Hence $R_{0} \subset R^{\text {re }}-R^{\text {re }}$ if and only if $R_{0} \subset \Lambda_{\text {diff. }}$

\subsection{Affine forms}

Our definition of affine reflection systems follows the practice of [14] in defining root systems without reference to a bilinear form. In the literature, it is customary to define affine root systems and their generalizations, the extended affine root systems (EARS), in real vector spaces using semidefinite forms. In Proposition 5.4 we will give a characterization of affine reflections systems in terms of affine invariant forms where, by definition, an affine form for a pre-reflection system $(R, X)$ over $\mathbb{K}$ is an invariant form $b$ satisfying $R^{\mathrm{im}}=R \cap \operatorname{Rad} b$. In particular, affine forms are strictly invariant in the sense of $\S 2.7$. For example, the forms used in the theory of EARS are affine forms in our sense.

Lemma 5.3. Let $(R, X)$ be pre-reflection system admitting an affine form $b$.

(a) $(R, X)$ is of the type considered in $\$ 2.9$ with respect to the form $b=(\mid)$. In particular, $R$ is a reflection system. 
(b) If $\operatorname{Re}(R)$ is connected, $b$ is unique up to a nonzero scalar.

(c) Let $Y=X / \operatorname{Rad} b$, let $f: X \rightarrow Y$ be the canonical map and put $S=f(R)$. Then there exists a unique reflection map $s: S \rightarrow \operatorname{Ref}(Y)$ such that $(S, Y, s)$ is a reflection system and $f$ is a morphism of reflection systems. Moreover, $S$ and $f$ have the following properties:

(i) $f$ is an extension.

(ii) The form $b_{Y}: Y \times Y \rightarrow \mathbb{K}$, defined by $b_{Y}\left(f(x), f\left(x^{\prime}\right)\right)=b\left(x, x^{\prime}\right)$ for $x, x^{\prime} \in X$, is a nondegenerate invariant form for $(S, Y, s)$.

Proof. (a) By (2.18) and the definition of an affine form, $R^{\text {re }}=\{\alpha \in R$ : $b(\alpha, \alpha) \neq 0\}$. Then (a) is immediate from (2.16) and $\$ 2.9$.

(b) Let $b^{\prime}$ be another affine form. Since $b\left(X, R^{\mathrm{im}}\right)=0=b^{\prime}\left(X, R^{\mathrm{im}}\right)$ it is sufficient to prove the existence of $c \in \mathbb{K}^{\times}$such that $b^{\prime}(x, \alpha)=c b(x, \alpha)$ holds for all $\alpha \in R^{\text {re }}$. In view of (2.16) this is in turn equivalent to

$$
b^{\prime}(\alpha, \alpha)=c b(\alpha, \alpha) \quad \text { for all } \alpha \in R^{\mathrm{re}} .
$$

There obviously exists $c \in \mathbb{K}^{\times}$such that (5.11) holds for some $\alpha_{0} \in R^{\text {re }}$. Connectedness of $\operatorname{Re}(R)$ then implies that (5.11) holds for all $\alpha \in R^{\text {re }}$.

(c) The form $b_{Y}$ is obviously well-defined. Also, for $\alpha \in R^{\text {re }}$ we have $f(\alpha) \neq 0$ and $b(\alpha, \alpha) \neq 0$ whence $\left\langle x, \alpha^{\vee}\right\rangle=2 b(x, \alpha) / b(\alpha, \alpha)$ for all $x \in X$. If $s: S \rightarrow$ $\operatorname{Ref}(Y)$ exists as claimed, then necessarily

$$
\left\langle f(x), f(\alpha)^{\vee}\right\rangle=\left\langle x, \alpha^{\vee}\right\rangle=2 \frac{b(x, \alpha)}{b(\alpha, \alpha)}=2 \frac{b_{Y}(f(x), f(\alpha))}{b_{Y}(f(\alpha), f(\alpha))}
$$

for $x \in X$. Conversely, let $s$ be the reflection map defined in (2.22) for $\Phi=$ $f\left(R^{\mathrm{re}}\right)=S^{\times}$and $(\mid)=b_{Y}$, i.e., $f(\alpha)^{\vee}$ is given by (5.12). Then it follows from (2.8) that $f \circ s_{\alpha}=s_{f(\alpha)} \circ f$ holds for all $\alpha \in R$, which proves that $(S, Y, s)$ is a reflection system and at the same time that $f$ is a morphism. The remaining assertions are now clear.

Proposition 5.4. Let $(R, X)$ be a pre-reflection system. Then $(R, X)$ is an affine reflection system if and and only if it satisfies the following conditions:

(i) $(R, X)$ is integral,

(ii) $(R, X)$ has an affine form, and

(iii) $\left\langle R, \alpha^{\vee}\right\rangle$ is bounded, for every $\alpha \in R^{\mathrm{re}}$. 
In this case:

(a) Let $b$ be an affine form for $(R, X)$ and let $f: X \rightarrow X / \operatorname{Rad} b$ be the canonical map. Then $(S, Y)=(f(R), X / \operatorname{Rad} b)$ is the quotient root system of $R$ and $f$ its canonical projection. Moreover, $\operatorname{Re}(R)$ is connected if and only if $S$ is irreducible.

(b) There exists a unique affine form ( | )a that is normalized in the sense of (2.20), i.e., for every connected component $C$ of $\operatorname{Re}(R)$ we have

$$
2 \in\left\{(\alpha \mid \alpha)_{a}: \alpha \in C^{\times}\right\} \subset\{2,3,4,6,8\} .
$$

The normalized form ( | ) a satisfies

$$
\left\{(\alpha \mid \alpha)_{a}: \alpha \in C^{\times}\right\} \in\{\{2\},\{2,4\},\{2,6\},\{2,8\},\{2,4,8\}\} .
$$

Its radical is $\operatorname{Rad}(\mid)_{a}=\operatorname{Ker} f$. If $\mathbb{K}=\mathbb{R}$ then $(\mid)_{a}$ is positive semidefinite.

Proof. Let $(R, X)$ be an affine reflection system with quotient root system $S$ and canonical projection $f$. Then (i) and (iii) follow from Lemma 4.5 (a), (4.8) and the corresponding properties of $S$. By Proposition 2.2 the root system $S$ has a unique normalized invariant form $(\mid)$. Let $(\mid)_{a}$ be the pull back of $(\mid)$ to an invariant form on $X$ as defined in Lemma 4.1 (e). Since ( | ) is nondegenerate, we have $R \cap \operatorname{Rad}(\mid)_{a}=R \cap \operatorname{Ker}(f)=R_{0} \subset R^{\mathrm{im}}$ by (4.7). On the other hand, $f\left(R^{\mathrm{im}}\right)=S^{\mathrm{im}}=\{0\}$, whence $R^{\mathrm{im}} \subset R_{0}$ and so ( $\left.\mid\right)_{a}$ is an affine form.

Conversely, suppose (i)-(iii) hold. By Lemma $5.3(\mathrm{a}),(R, X)$ is a reflection system. Let $(S, Y)$ and $f: X \rightarrow Y$ be the reflection system and extension constructed in Lemma 5.3 (c). Note that $S$ is integral by Lemma 4.5 (a). Also $\left\langle S, \xi^{\vee}\right\rangle$ is bounded for every $\xi \in S$. Hence, by Proposition 2.2, $S$ is a locally finite root system, and consequently $R$ is an affine reflection system with quotient root system $S$ and canonical projection $f$. The last part of (a) follows from (4.12).

(b) The form $(\mid) a$ constructed above is normalized since $(\mid)$ is normalized and $f$ maps connected components onto connected components of $S$, see Lemma 4.5 (d.i). Uniqueness of ( | $)_{a}$ follows from uniqueness of ( | ) on $S$. The remaining statements all follow from Proposition 2.2.

Remark. Since the reflective roots of an affine reflection system $R$ are given by $R^{\mathrm{re}}=\left\{\alpha \in R:(\alpha \mid \alpha)_{a} \neq 0\right\}$, they are also called the anisotropic roots.

Corollary 5.5. A pre-reflection system over the reals is affine if and only if it is integral and has a positive semidefinite affine form. 
Proof. If $R$ is a real affine reflection system, its normalized affine form $(\mid)_{a}$ is positive semidefinite by Proposition 5.4. For the converse, let $b$ be a positive semidefinite affine form for $R$. We follow the proof of Proposition 5.4 and consider $Y=X / \operatorname{Rad} b, f: X \rightarrow Y$ the canonical map and $S=f(R)$. It then remains to show that $S$ is a locally finite root system. But this follows from [26, Theorem 4.2(b)].

\subsection{Examples and Remarks}

(a) As usual, the rank of a reflection system $(R, X)$ is defined as $\operatorname{rank}(R, X)=$ $\operatorname{dim} X$.

Let $R$ be an affine reflection system over $\mathbb{K}=\mathbb{R}$ of finite rank. We will say that $R$ is discrete if $R$ is a discrete subset of $X$. In case $R$ has finite rank, $\operatorname{Re}(R)$ is connected and $R_{0} \subset \Lambda_{\text {diff }}$, it is easily seen that $R$ is discrete if and only if $\mathbb{Z}[R]$ is a lattice in $X$.

(b) Let $(R, X)$ be an affine reflection system over $\mathbb{K}=\mathbb{R}$ with the following properties: $R$ has finite rank, $\operatorname{Re}(R)$ is connected, $R=-R$ and $R$ is discrete. Then $R$ is called

- an EARS, an abbreviation of "extended affine root system", if $R$ is reduced, tame (see Corollary $5.2(\mathrm{c})$ ), and all root strings are unbroken;

- a SEARS, an abbreviation of "Saito's extended affine root system", if $R=$ $\operatorname{Re}(R)$.

That our definition of an EARS is equivalent to the one given by Azam, Allison, Berman, Gao and Pianzola in [1, II, Definition 2.1] is a consequence of Lemma 4.5 (e). In particular, by Corollary 5.2(b), every EARS is a partial root system. That our definition of a SEARS is equivalent to Saito's definition of an "extended affine root system" in [37] follows from Corollary 5.5.

It was shown in [6, Theorem 18] that every reduced SEARS can be uniquely extended to an EARS and, conversely, the reflective roots of an EARS are the non-zero roots of a SEARS. This is now immediate from our results. Indeed, by Corollary 5.2 an affine reflection system is tame and has unbroken root strings if and only $R_{0}=\Lambda_{\text {diff. }}$.

Weyl groups of extended affine root systems are studied in [7, 10, 19].

(c) In [29], Morita and Yoshii define a LEARS, an abbreviation of a "locally extended affine root system". In our terminology, this is a symmetric affine reflection system $R$ over $\mathbb{K}=\mathbb{R}$ such that $R=\operatorname{Re}(R)$ is connected. The equivalence of this definition with the one in [29] follows from Corollary 5.5. 
(d) In [8], Azam defines a GRRS, an abbreviation of a "generalized reductive root system". In our terminology, this is a symmetric real reduced, discrete affine reflection system $R$ which has finite rank and unbroken root strings.

We point out that our description of affine reflection systems in $\$ 5.1$ applies to EARS, SEARS, LEARS and GRRS. In particular, it generalizes the structure theorem of extended affine root systems proven in [1, II, Theorem 2.37] and [29, Proposition 4.2].

(e) Lie algebras whose root systems (in the appropriate sense) are EARS have been studied in [1, 2, 3, 4, 46, 47, 45]. For SEARS see [38, 44], for LEARS see [29] and for GRRS see [9, 8]. In particular, it is shown in [1, I, Theorem 2.16] that the root system of an extended affine Lie algebra is an EARS. A special case of an EARS is the root system of an affine Kac-Moody algebra. We also mention that the notion of tameness comes in fact from the theory of extended affine Lie algebras, where tameness of the Lie algebra is expressed by tameness of the corresponding root system [2, Lemma 3.62].

Lie algebras whose root system is a symmetric reduced affine reflection system over an arbitrary $\mathbb{K}$ appear in [35] and [34].

Lie superalgebras with a grading by an affine reflection system of arbitrary rank but with a 3-graded quotient root system are described in [17].

Acknowledgments. During a large part of the preparation of this paper, the firstnamed author was a guest of the Department of Mathematics and Statistics of the University of Ottawa. The hospitality of this institution is gratefully acknowledged.

The authors thank Karl-Hermann Neeb for useful comments on a previous version of this paper.

\section{Bibliography}

[1] B. Allison, S. Azam, S. Berman, Y. Gao and A. Pianzola, Extended affine Lie algebras and their root systems, Mem. Amer. Math. Soc. 126 (1997), no. 603, x+122.

[2] B. Allison, S. Berman and A. Pianzola, Covering algebras I. Extended affine Lie algebras, J. Algebra 250 (2002), no. 2, 485-516.

[3] Covering algebras II. Isomorphism of loop algebras, J. Reine Angew. Math. 571 (2004), 39-71.

[4] B. Allison and Y. Gao, The root system and the core of an extended affine Lie algebra, Selecta Math. (N.S.) 7 (2001), no. 2, 149-212.

[5] S. Azam, Nonreduced extended affine root systems of nullity 3, Comm. Algebra 25 (1997), no. 11, 3617-3654. 
[6] __ Extended affine root systems, J. Lie Theory 12 (2002), 515-527.

[7] _ Nonreduced extended affine Weyl groups, J. Algebra 269 (2003), no. 2, 508-527. , Generalized reductive Lie algebras: connections with extended affine Lie algebras and Lie tori, Canad. J. Math. 58 (2006), no. 2, 225-248.

[9] S. Azam, S. Berman and M. Yousofzadeh, Fixed point subalgebras of extended affine Lie algebras, J. Algebra 287 (2005), no. 2, 351-380.

[10] S. Azam and V. Shahsanaei, Presentation by conjugation for $\mathrm{A}_{1}$-type extended affine Weyl groups, J. Algebra 319 (2008), no. 5, 1932-1953.

[11] N. Bardy, Systèmes de racines infinis, Mém. Soc. Math. Fr. (N. S.) 65 (1996), 1-188.

[12] _ Définition abstraite d'un système de racines dans le cas symétrisable, J. Algebra 271 (2004), no. 1, 108-178.

[13] N. Bourbaki, Algèbre, chapitres 4 à 7, Masson, Paris, 1981.

[14] _ Groupes et algèbres de Lie, chapitres 4-6, Masson, Paris, 1981.

[15] F. Bruhat and J. Tits, Groupes réductifs sur un corps local, Inst. Hautes Études Sci. Publ. Math. 41 (1972), 5-251.

[16] V. V. Deodhar, On the root system of a Coxeter group, Comm. Algebra 10 (1982), 611-630.

[17] E. García and E. Neher, Tits-Kantor-Koecher superalgebras of Jordan superpairs covered by grids, Comm. Algebra 31 (2003), no. 7, 3335-3375.

[18] J-Y. Hée, Systèmes de racines sur un anneau commutatif totalement ordonné, Geom. Dedicata 37 (1991), no. 1, 65-102.

[19] G. Hofmann, Weyl Groups with Coxeter Presentation and Presentation by Conjugation, J. Lie Theory 17 (2007), no. 2, 337-355.

[20] J. Humphreys, Reflection Groups and Coxeter Groups, Cambridge Studies in Advanced Mathematics 29, Cambridge University Press, Cambridge, 1990.

[21] N. Jacobson, Basic Algebra, Volume 1, W. H. Freeman and Co., 1974.

[22] V. Kac, Lie superalgebras, Adv. in Math. 26 (1977), no. 1, 8-96.

[23] _ Infinite Dimensional Lie Algebras, Third Edition, Cambridge University Press, 1990.

[24] S. Kumar, Kac-Moody Groups, Their Flag Varieties and Representation Theory, Progress in Mathematics 204, Birkhäuser Boston Inc., Boston, MA, 2002.

[25] B. Kürner and K.-H. Neeb, Invariant symmetric bilinear forms for reflection groups, J. Geom. 71 (2001), no. 1-2, 99-127. 
[26] O. Loos and E. Neher, Locally finite root systems, Mem. Amer. Math. Soc. 171 (2004), no. 811, x+214.

[27] É. Lucas, Théorie des nombres, Volume 1, Albert Blanchard, Paris, 1961 (reprint of the original 1891 edition).

[28] R. V. Moody and A. Pianzola, Lie Algebras with Triangular Decompositions, Canad. Math. Soc. series of monographs and advanced texts, John Wiley, 1995.

[29] J. Morita and Y. Yoshii, Locally extended affine Lie algebras, J. Algebra 301 (2006), no. $1,59-81$.

[30] K.-H. Neeb, Holomorphic highest weight representations of infinite dimensional complex classical groups, J. Reine Angew. Math. 497 (1998), 171-222.

[31] K.-H. Neeb and N. Stumme, The classification of locally finite split simple Lie algebras, J. Reine Angew. Math. 533 (2001), 25-53.

[32] E. Neher, Systèmes de racines 3-gradués, C. R. Acad. Sci. Paris Sér. I 310 (1990), 687-690.

[33] _ Lie algebras graded by 3-graded root systems and Jordan pairs covered by a grid, Amer. J. Math. 118 (1996), 439-491.

[34] _ Extended affine Lie algebras, C. R. Math. Acad. Sci. Soc. R. Canada 26 (2004), no. 3, 90-96.

[35] , Lie tori, C. R. Math. Acad. Sci. Soc. R. Canada 26 (2004), no. 3, 84-89.

[36] B. Rémy, Groupes de Kac-Moody déployés et presque déployés, Astérisque 277, Soc. Math. France, Montrouge, 2002, viii+348.

[37] K. Saito, Extended affine root systems. I. Coxeter transformations, Publ. Res. Inst. Math. Sci. 21 (1985), no. 1, 75-179.

[38] K. Saito and D. Yoshii, Extended affine root system. IV. Simply-laced elliptic Lie algebras, Publ. Res. Inst. Math. Sci. 36 (2000), no. 3, 385-421.

[39] V. Serganova, On generalizations of root systems, Comm. Algebra 24 (1996), no. 13, 4281-4299.

[40] R. Steinberg, Lectures on Chevalley Groups, Yale University Lecture Notes, New Haven, Conn., 1967.

[41] N. Stumme, The structure of locally finite split Lie algebras, J. Algebra 220 (1999), 664-693.

[42] J. Tits, Moufang octagons and the Ree groups of type ${ }^{2} F_{4}$, Amer. J. Math. 105 (1983), no. 2, 539-594.

[43] $\ldots$, Uniqueness and presentation of Kac-Moody groups over fields, J. Algebra 105 (1987), 542-573. 
[44] H. Yamane, A Serre-type theorem for the elliptic Lie algebras with rank $\geq 2$, Publ. Res. Inst. Math. Sci. 40 (2004), no. 2, 441-469.

[45] Y. Yoshii, Coordinate algebras of extended affine Lie algebras of type $A_{1}$, J. Algebra 234 (2000), no. 1, 128-168.

[46] _ Root-graded Lie algebras with compatible grading, Comm. Algebra 29 (2001), no. 8, 3365-3391.

[47] Classification of division $\mathbb{Z}^{n}$-graded alternative algebras, J. Algebra 256 (2002), no. 1, 28-50.

[48] _ Root systems extended by an abelian group and their Lie algebras, J. Lie Theory 14 (2004), no. 2, 371-394.

Received May 18, 2009; revised May 28, 2009.

\section{Author information}

Ottmar Loos, Fakultät für Mathematik und Informatik, FernUniversität in Hagen, 58097 Hagen, Germany.

E-mail: Ottmar.Loos@FernUni-Hagen.de

Erhard Neher, Department of Mathematics and Statistics, University of Ottawa, Ottawa, Ontario K1N 6N5, Canada.

E-mail: neher@uottawa.ca 